\title{
InVENTARIO FLORÍSTICO dE LA CAÑADA La ChaCONA-JUAN Crispín y zOnAs adyaCentes, Depresión Central DE CHIAPAS, MÉXICO
}

\author{
Josefa Anahi Espinosa-Jiménez ${ }^{1}$, Angelita López-Cruz, Miguel Ángel Pérez-Farrera y Sergio López \\ Herbario Eizi Matuda, Facultad de ciencias biológicas, Universidad de Ciencias y Artes de Chiapas, \\ Tuxtla Gutiérrez, Chiapas, México \\ 1Autor para la correspondencia:anahi_espinosa_jimenez@yahoo.com.mx
}

\begin{abstract}
Resumen: Se presenta un listado florístico de la Cañada La Chacona-Juan Crispín, Chiapas, dentro de los municipios de San Fernando, Tuxtla Gutiérrez y Berriozábal, Chiapas. Se reportan 642 especies y 31 infraespecies, agrupadas en 107 familias y 412 géneros; en bosque tropical caducifolio, bosque tropical subcaducifolio y bosque de Quercus. El bosque tropical caducifolio fue el de mayor riqueza. Las familias mejor representadas fueron Fabaceae, Asteraceae y Euphorbiaceae. Tillandsia, Eugenia y Euphorbia fueron los géneros con un mayor número de especies. Las hierbas conformaron la forma de crecimiento más abundante (34.4\%). Se encontraron 13 especies endémicas a Chiapas y 19 de distribución restringida. Quince especies están en alguna categoría de riesgo dentro de la Norma Oficial Mexicana (NOM-059-SEMARNAT-2010), y 12 se encuentran en la Lista Roja de la Unión Internacional para la Conservación de la Naturaleza. Aunque el grado de perturbación de la zona es alto, el área es importante por su riqueza de especies y presencia de endemismos.
\end{abstract}

Palabras clave: conservación, endemismo, florística, riqueza, vegetación.

\begin{abstract}
A floristic study for Cañada La Chacona-Juan Crispin, Chiapas is given. 642 species and 31 infraspecies classified in 107 families and 412 genera were collected in the municipalities San Fernando, Tuxtla Gutiérrez, and Berriozábal, Chiapas, Mexico. The vegetation types were tropical deciduous forest, subdeciduous tropical forest, and Quercus forest, with the highest species richness in tropical deciduous forest. Fabaceae, Asteraceae, and Euphorbiaceae were the best represented families. Tillandsia, Eugenia, and Euphorbia were the genera with the higher number of species. Herbs were the most abundant growth form (34.4\%). Thirteen species were endemic to Chiapas and 19 species had a restricted distribution. Fifteen species are listed in the Norma Oficial Mexicana (NOM-059-SEMARNAT-2010), and 12 species are in the IUCN Red List. Although the level of disturbance in the area is high, the area is important due to its species richness and endemism.
\end{abstract}

Key words: conservation, endemism, floristics, richness, vegetation.

$\mathbf{M}$ éxico es uno de los países biológicamente más diversos, con altos porcentajes de especies endémicas y en peligro de extinción (Toledo y Ordóñez, 1998; Ceballos, 2007). Gracias a su ubicación geográfica, a su geología y topografía, el país presenta en su territorio una alta variedad en su flora y tipos de vegetación, que van desde selvas altas hasta la vegetación de las zonas áridas (Rzedowski, 2006; Miranda y Hernández X., 1963). Entre los tipos de vegetación más diversos, poco estudiados y de poca representatividad en áreas naturales protegidas, se encuentra el Bosque Tropical Caducifolio (BTC) (Ceballos et al., 2010a). Gordon et al. (2006) mencionan que, hace algunos años, se estimó que en el país persistía en remanentes sólo un 27\% de la cobertura original del BTC; además de un porcentaje similar que permanecía en estado de alta degradación.

La Cañada La Chacona-Juan Crispín presenta una vegetación de tipo BTC (Rzedowski, 2006), también conocido como Selva Baja Caducifolia (Miranda y Hernández X., 1963), o selvas secas (Dirzo y Ceballos, 2010). La región se encuentra sometida a fuertes presiones demográficas que resultan en la destrucción del hábitat para construcción de solares y viviendas, además del pastoreo y extracción de material para construcción. Este escenario ha sido documentado por Ceballos et al. (2010b), quienes comentan que la selva 
seca de la Depresión Central de Chiapas se encuentra entre las más amenazadas del mundo debido al alto grado de alteración o destrucción. A pesar de la problemática, las exploraciones botánicas y estudios han sido pocos; por ejemplo, Reyes-García y Sousa (1997) realizaron un inventario florístico de la Selva Baja Caducifolia de la Depresión Central de Chiapas, en el cual registraron 989 especies, e incluyen sitios de colecta de la cañada "La Chacona"; Gallegos-Ramos (2009) generó un inventario florístico de la cañada "La Chacona" y la parte occidente del Parque Nacional Cañón del Sumidero, donde encontró 549 especies y, Espinosa-Jiménez et al. (2011) reportaron 1,381 especies en un trabajo de inventario en el Parque Nacional Cañón del Sumidero.
Gordon et al. (2006) mencionan que es inminente el desarrollo de programas de conservación en BTC, aunque también comentan que uno de los obstáculos para la realización de esta tarea es la falta de inventarios biológicos para las zonas con este tipo de vegetación. El inventario florístico que aquí se presenta constituye un primer esfuerzo para construir una estrategia encaminada a la conservación de la zona de estudio.

\section{Materiales y métodos}

Zona de estudio. La Chacona era un Rancho ubicado en la colonia Juan Crispín, ejido plan de Ayala, en la Ciudad de

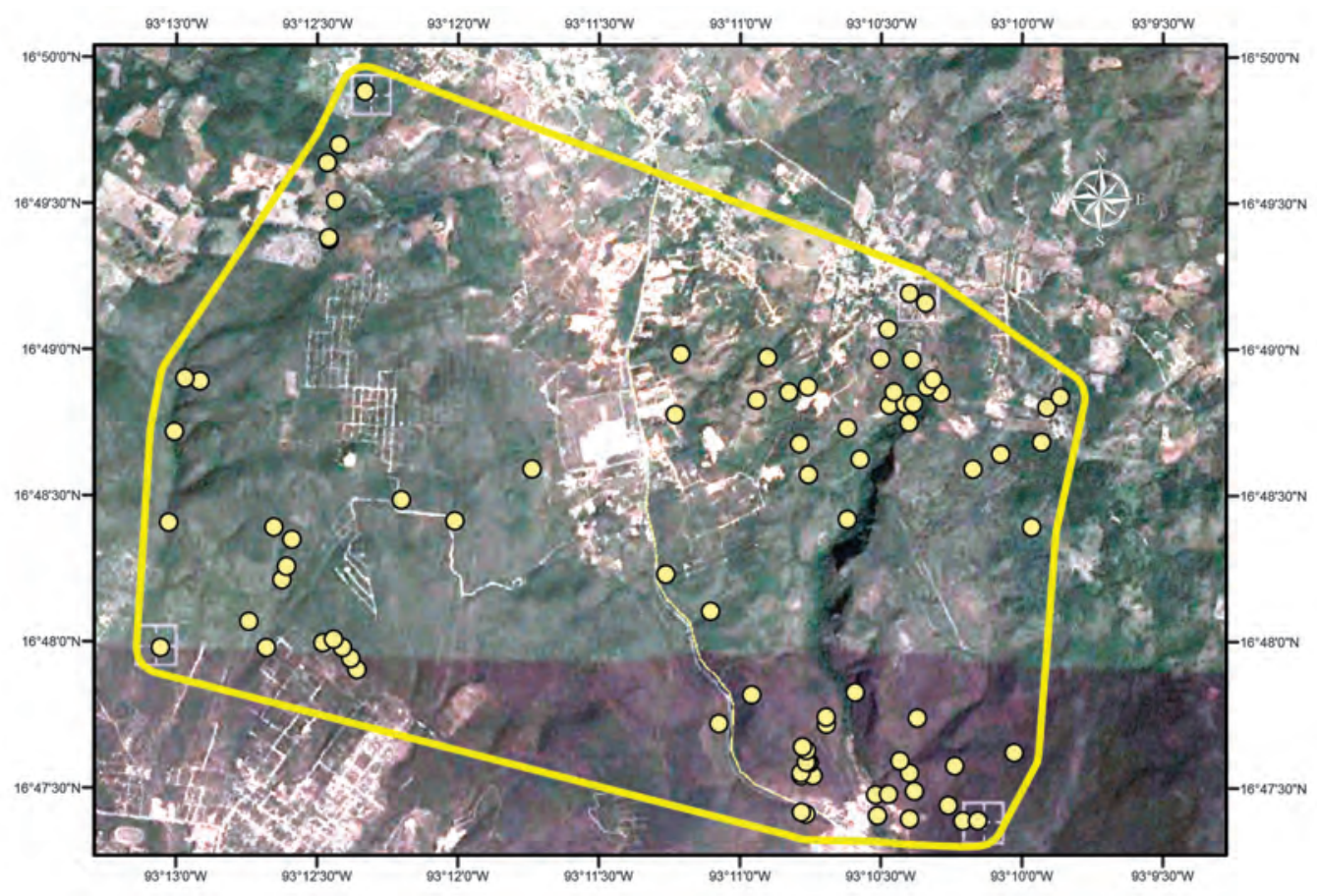

\section{Leyendas}

Puntos de colecta Área de muestreo

O

\section{Parámetros cartográficos}

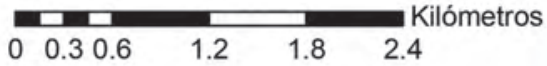

Escala 1:40,000

Proyección: Universal Transversal de Mercator-15N

\section{Esferoide:}

$$
\text { Clarke } 1866
$$

Datum: WGS1984 - Zona $15 \mathrm{~N}$

\section{Localización}

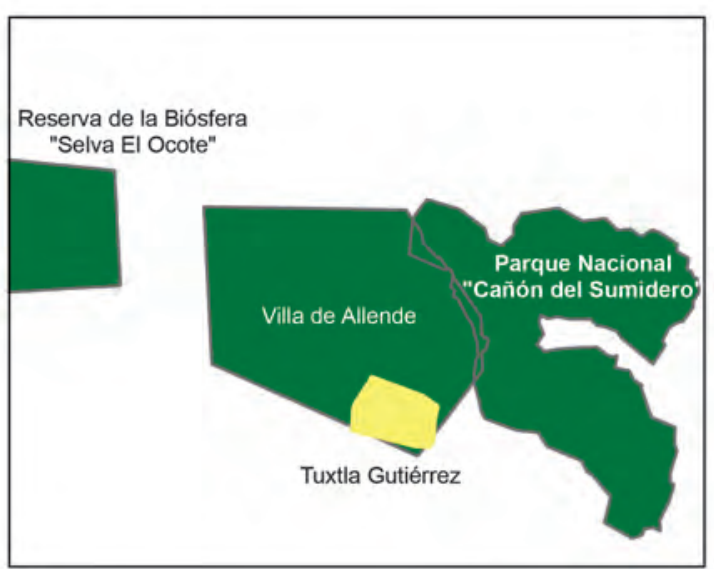

Figura 1. Área de estudio 
Tuxtla Gutiérrez. En la actualidad es un vivero de la SAGARPA, y se encuentra en la base de la Cañada Chacona y la Cañada Aguacate. Por la Cañada Chacona va actualmente la carretera Juan Crispín-Chicoasén (Andrade de Sousa et al., 2007). El área se encuentra dentro de la Depresión Central de Chiapas, colinda con la parte suroeste de la Region Terrestre Prioritaria (RTP)-141 La Chacona-Cañón del Sumidero (Arriaga, 2009), y se halla dentro de los terrenos forestales de Villa de Allende, sitio decretado como Zona Protectora Forestal en 1939, después de dar a conocer los servicios ecosistémicos que ofrece en cuanto a regulación de condiciones climatológicas (Vargas et al., 2000).

Las exploraciones y recolectas botánicas se llevaron a cabo dentro de la Cañada La Chacona y zonas aledañas dentro de los municipios de San Fernando, Berriozábal y Tuxtla Gutiérrez. El tipo de vegetación que domina el paisaje, de acuerdo con la clasificación de Rzedowski (2006), es BTC, también se registran, con una menor extensión, Bosque Tropical Subcaducifolio (BTSC) y Bosque de Quercus (BQ). El BTC se desarrolla sobre relieve poco accidentado, es el tipo de vegetación más afectado por las actividades agrosilvopastoriles; el BTSC se encuentra sobre terrenos accidentados, frecuente presencia de rocas y, en ocasiones, inaccesibles. El BQ está representado por dos tipos de formaciones que se limitan a pequeños fragmentos relictuales perturbados, como consecuencia de prácticas de extracción de arena y grava.

Los límites de la zona de estudio son: $16^{\circ} 49^{\prime} 57.2^{\prime \prime} \mathrm{N}$, $93^{\circ} 13^{\prime} 8.5^{\prime \prime} \mathrm{O}, 16^{\circ} 47^{\prime} 17.3^{\prime \prime} \mathrm{S}$ y $93^{\circ} 09^{\prime} 46.5^{\prime \prime} \mathrm{E}$, con una superficie de 2,069.90 ha (Figura 1), de 594 a $1,030 \mathrm{~m}$ s.n.m. Presenta una temperatura media anual de $17.9^{\circ} \mathrm{C}$ y la precipitación anual es de 1,746 mm (González-Espinosa et al., 2005). INEGI (1985a) registra suelos de tipo litosol más rendzina de textura media; luvisol crómico más regosol eútrico de textura fina; regosol eútrico de textura gruesa y feozem lúvico, luvisol órtico más litosol de textura fina. Geológicamente, la zona pertenece al Cretácico superior y Eoceno constituidos principalmente por roca caliza, lutita, arenisca y conglomerados (INEGI, 1985b).

Recolectas. Se realizaron 36 salidas de campo de enero a septiembre de 2010. Se eligieron los sitios con menor grado de disturbio. Los puntos de recolecta se establecieron con ayuda del programa Google Earth versión 5.0 para Windows. Las coordenadas geográficas de los sitios de muestreo se ingresaron al programa ArcGis versión 9.1 para la construcción del mapa y para obtener la superficie del área de recolecta. La recolecta y el procesamiento de ejemplares se realizaron según el método propuesto por Lot y Chiang (1986). Se recolectaron cuatro duplicados para cada ejemplar, con excepción de la familia Orchidaceae, para la cual se recolectó una muestra debido a los estrictos requerimientos en su establecimiento, desarrollo y reproducción. Los herbarios en donde se depositaron los ejemplares fueron:
Herbario Eizi Matuda (HEM), el herbario de la Secretaría del Medio Ambiente e Historia Natural (CHIP), Herbario Nacional (MEXU), Missouri Botanical Garden (MO) y Herbario de la Académia de Ciencias de California (CAS). Para la identificación a nivel de especie se consultaron literatura especializada y a especialistas en algunas familias. La base de datos se completó con los ejemplares registrados en la REMIB (Red Mundial de Información sobre Biodiversidad; CONABIO, 2008a) y el CAS (2012). Las autoridades botánicas se tomaron de sitio de trópicos (www.tropicos.org). Toda la información de las colectas realizadas y consultadas se integró en una base en Microsoft Access 2007. La clasificación para los helechos se basó en el sistema de Crabbe et al. (1975) modificado por Mickel y Smith (2004); y para las angiospermas, APG III (APG, 2009). Para dar a conocer el número de especies en riesgo que alberga el área de estudio se consultaron la NOM-059-SEMARNAT-2010 (SEMARNAT, 2010) y la Lista Roja de la Unión Internacional para la Conservación de la Naturaleza (IUCN, 2010). Se consideraron como endémicas sólo las especies cuya distribución está limitada al estado de Chiapas y de distribución restringida a aquellas registradas en el sur de México hasta Guatemala. La distribución de las especies se estableció con base en la revisión de floras, monografías y bases de datos del MO (2012), apoyada por las bases del CAS (2012) y KEW (Royal Botanic Gardens, 2012). Los tipos de vegetación se basaron en la clasificación de Rzedowski (2006). Las formas de crecimiento contenidas en el apéndice se tomaron del catálogo de términos botánicos utilizado en el sistema Biótica 5.0 (CONABIO, 2008b).

\section{Resultados}

Flora. Se obtuvo una base de datos con 1,212 registros, que incluye 642 especies y 31 infraespecies, agrupadas en 107 familias y 412 géneros (Cuadro 1). El grupo mejor representado fue el de las Eudicotiledóneas con el 82.3\% del total de las especies, seguido de las Monocotiledóneas con $12.1 \%$, mientras que los grupos de menor representación fueron Pteridophyta y Gymnospermae con $3.1 \%$ y $0.15 \%$, respec-

Cuadro 1. Distribución en grupos taxonómicos de las plantas vasculares del área de estudio.

\begin{tabular}{lcccc}
\hline Grupos & Familias & Géneros & Especies & Infraespecies \\
\hline Pteridophyta & 7 & 11 & 20 & 1 \\
Gymnospermae & 1 & 1 & 1 & 0 \\
Angiospermae & & & & \\
$\quad$ Magnolidas & 4 & 9 & 14 & 0 \\
$\quad$ Monocotiledóneas & 13 & 50 & 78 & 7 \\
$\quad$ Eudicotiledóneas & 82 & 341 & 529 & 23 \\
Total & $\mathbf{1 0 7}$ & $\mathbf{4 1 2}$ & $\mathbf{6 4 2}$ & $\mathbf{3 1}$ \\
\hline
\end{tabular}


Cuadro 2. Lista de especies endémicas y bajo alguna categoría de riesgo en la NOM-059-SEMARNAT-2010, NOM-A: Amenazada, NOM-Pr: Sujetas a protección especial, NOM-P: En peligro de extinción; y en la lista roja de la IUCN, IUCN-EN: En peligro (Endangered); IUCN-LC: Preocupación menor (Least concern), IUCN-VU: Vulnerable.

\begin{tabular}{|c|c|c|c|}
\hline Especies & Familia & Categoría de riesgo & Distribución \\
\hline Holographis parayana & Acanthaceae & ninguna & endémica \\
\hline Agave grijalvensis & Agavaceae & NOM-Pr & endémica \\
\hline Astronium graveolens & Anacardiaceae & NOM-A & no endémica \\
\hline Brahea nitida & Araceae & IUCN-VU; NOM-Pr & no endémica \\
\hline Gymnolaena chiapasana & Asteraceae & ninguna & endémica \\
\hline Carpinus caroliniana & Betulaceae & NOM-A & no endémica \\
\hline Catopsis berteroniana & Bromeliaceae & NOM-Pr & no endémica \\
\hline Pitcairnia breedlovei & Bromeliaceae & ninguna & endémica \\
\hline Pitcairnia chiapensis & Bromeliaceae & ninguna & endémica \\
\hline Cephalocereus nizandensis & Cactaceae & NOM-Pr & endémica \\
\hline Pterocereus gaumeri & Cactaceae & NOM-P & no endémica \\
\hline Maytenus matudae & Celastraceae & IUCN-VU & no endémica \\
\hline Callisia gentlei var. macdougallii & Commelinaceae & ninguna & endémica \\
\hline Bernardia mollis & Euphorbiaceae & NOM-A & no endémica \\
\hline Croton guatemalensis & Euphorbiaceae & NOM-Pr & no endémica \\
\hline Euphorbia pseudofulva & Euphorbiaceae & ninguna & endémica \\
\hline Conzattia chiapensis & Fabaceae & ninguna & endémica \\
\hline Inga chiapensis & Fabaceae & UCN-VU & no endémica \\
\hline Lonchocarpus martinezii & Fabaceae & ninguna & endémica \\
\hline Lonchocarpus minimiflorus & Fabaceae & IUCN-EN & no endémica \\
\hline Dendrosida batesii & Malvaceae & NOM-A & endémica \\
\hline Robinsonella mirandae & Malvaceae & IUCN-VU & no endémica \\
\hline Robinsonella pilosissima & Malvaceae & ninguna & endémica \\
\hline Robinsonella samaricarpa & Malvaceae & IUCN-VU & no endémica \\
\hline Cedrela odorata & Meliaceae & IUCN-VU; NOM-Pr & no endémica \\
\hline Swietenia humilis & Meliaceae & IUCN-VU & no endémica \\
\hline Eugenia breedlovei & Myrtaceae & ninguna & endémica \\
\hline Guarianthe skinneri & Orchidaceae & NOM-A & no endémica \\
\hline Serpocaulon triseriale & Polypodiaceae & NOM-A & no endémica \\
\hline Sideroxylon capiri & Sapotaceae & NOM-A & no endémica \\
\hline Taxodium mucronatum & Taxodiaceae & IUCN-LC & no endémica \\
\hline Guaiacum sanctum & Zygophyllaceae & IUCN-EN; NOM-A & no endémica \\
\hline
\end{tabular}

tivamente. Se encontaron 15 especies listadas en alguna categoría de riesgo dentro de la Norma Oficial Mexicana, de las cuales una está en peligro de extinción, ocho están como amenazadas y seis sujetas a protección especial. Para la IUCN, dos especies están en peligro de extinción, siete se hallan como vulnerables y una dentro de la categoría preocupación menor (Cuadro 2).

Se registraron 13 especies endémicas a Chiapas (Cuadro 2) y 19 de distribución restringida (Apéndice 1). De éstas, Pterocereus gaumeri (Chiapas, Yucatán) se considera en peligro de extinción dentro de la NOM-059-SEMARNAT2010, Bernardia mollis (Chiapas y Guatemala) se halla bajo la categoría de "Amenazada", mientras que Agave grijalvensis (Chiapas) y Cephalocereus nizandensis (Chiapas) se encuentran como sujetas a protección especial. Por otra parte, Inga chiapensis (Chiapas y Veracruz) y Maytenus matudae (Chiapas y Guatemala) están registradas como vulnerables en la Lista Roja de la IUCN.

Las familias con un mayor número de especies fueron $\mathrm{Fa}$ baceae (11.3\%), Asteraceae (7.4\%) y Euphorbiaceae (5.5\%). Tillandsia fue el género mejor representado con $1.5 \%$ del total, seguido de Eugenia y Euphorbia, ambas con 1.2\% (Cuadro 3). En formas de crecimiento, las hierbas fueron las más abundantes con $34.4 \%$, donde Asteraceae, Orchidaceae y Bromeliaceae fueron las familias que contribuyeron con un mayor número de especies. El 30.6\% estuvo representado por especies arbóreas; las familias con un mayor número de árboles fueron Fabaceae, Malvaceae y Euphorbiaceae. El $18 \%$ de las especies fueron arbustos, de los que Asteraceae, Fabaceae y Euphorbiaceae aportaron el mayor número de 


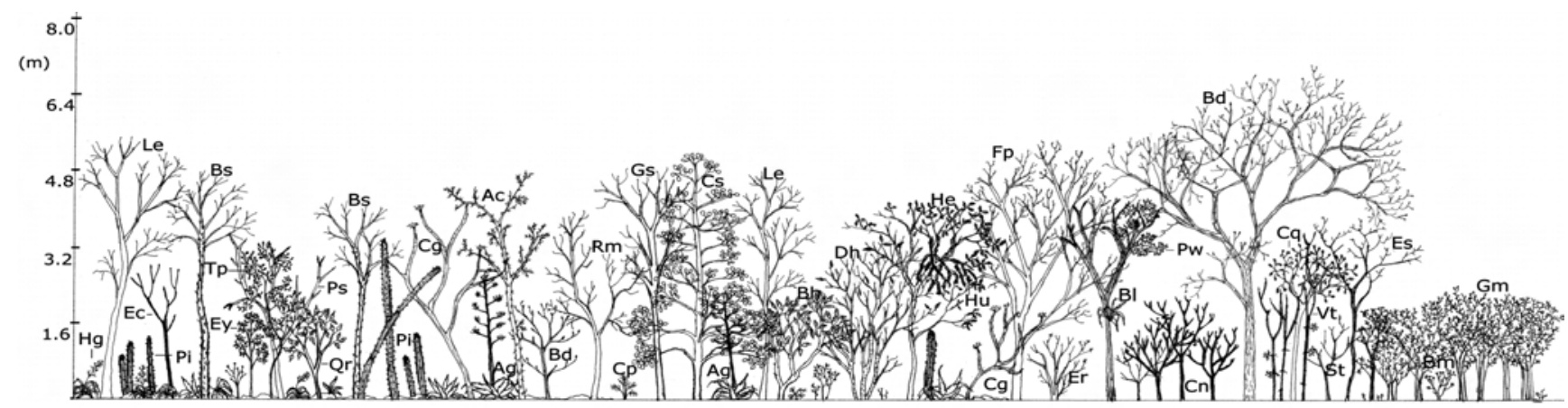

Figura 2. Esquema representativo del Bosque Tropical Caducifolio (BTC) del área de estudio. Ac. Acacia cornigera, Ag. Agave grijalvensis, Bh. Bourreria huanita, BI. Billbergia macrolepis, Bd. Terminalia buceras, Bm. Bonellia macrocarpa, Bs. Bursera simaruba, Cp. Chamaedorea plumosa, Cn. Cnidoscolus aconitifolius, Cs. Clusia salvinii, Cg. Comocladia guatemalensis, Dh. Dahnopsis americana, Er. Erythroxylum havanense, Ey. Eugenia yunckeri, Ec. Euphorbia calcarata, Es. Euphorbia schechtendalii, Fp. Ficus pertusa, Gm. Gymnopodium antigonoides, Gs. Guaiacum sanctum, He. Hauya elegans subsp. barcenae, Hg. Hechtia glomerata, Hu. Heliocereus undatus, Le. Leucaena leucocephala, Pw. Philodendron warscewiczii, Pi. Pilosocereus leucocephalus, Ps. Pseudobombax ellipticum, Qr. Quercus sp., Rm. Robinsonella mirandae, St. Stemmadenia pubescens, Tp. Tetrapterys sp., Vt. Vitis tiliifolia.

especies. Las lianas o bejucos contribuyeron con 15.5\%; esta forma de crecimiento estuvo bien representada por la familia Apocynaceae, seguida de Bignoniaceae y Fabaceae.

Vegetación. El BTC aportó la mayor proporción de especies, aproximadamente 58\% del total. De los 1,208 registros, $22.7 \%$ fueron colectados dentro de BTC y $29.1 \%$ en vegetación secundaria de BTC; el BTSC contribuyó con $9.7 \%$ del

Cuadro 3. Familias y géneros con mayor número de especies en la zona de estudio.

\begin{tabular}{lcc|cc}
\hline Familia & Géneros & $\begin{array}{c}\text { Especies, } \\
\text { infraespecies }\end{array}$ & Género & $\begin{array}{c}\text { Especies, } \\
\text { infraespecies }\end{array}$ \\
\hline Fabaceae & 40 & 73,7 & $\begin{array}{c}\text { Tillandsia } \\
\text { (Bromeliaceae) }\end{array}$ & 10,2 \\
Asteraceae & 43 & 48,3 & $\begin{array}{c}\text { Eugenia } \\
\text { (Myrtaceae) }\end{array}$ & 8,1 \\
Euphorbiaceae & 13 & 36,0 & $\begin{array}{c}\text { Euphorbia } \\
\text { (Euphorbiaceae) }\end{array}$ & 8 \\
Malvaceae & 19 & 31,2 & $\begin{array}{c}\text { Senna } \\
\text { (Fabaceae) }\end{array}$ & 7,1 \\
Apocynaceae & 17 & 27 & $\begin{array}{c}\text { Acalypha } \\
\text { (Euphorbiaceae) }\end{array}$ & 7 \\
Rubiaceae & 16 & 20,1 & $\begin{array}{c}\text { Adiantum } \\
\text { (Pteridaceae) }\end{array}$ & 7 \\
Bromeliaceae & 6 & 17,2 & $\begin{array}{c}\text { Bursera } \\
\text { (Burseraceae) }\end{array}$ & 7 \\
Orchidaceae & 13 & 16,1 & $\begin{array}{c}\text { Ficus } \\
\text { (Moraceae) }\end{array}$ & 7 \\
Solanaceae & 10 & 16 & $\begin{array}{c}\text { Solanum } \\
\text { (Solanaceae) } \\
\text { Dioscorea } \\
\text { (Dioscoreaceae) }\end{array}$ & 6,1 \\
\hline
\end{tabular}

total de ejemplares y $3.1 \%$ en vegetación secundaria, y el BQ con $5 \%$ y $9.1 \%$ recolectados en vegetación secundaria.

Caracterización de la vegetación. Las comunidades vegetales varían ampliamente en composición y estructura de acuerdo al grado de perturbación y en función a las presiones originadas por las actividades antropogénicas. Las descripciones y figuras de este apartado corresponden a comunidades vegetales con un grado mínimo de perturbación.

Bosque tropical caducifolio (BTC). Se desarrolla entre 500 y 1,000 m s.n.m. Se divide en dos estratos: arbóreo (2.55(-9) m) y herbáceo. Aunque existen elementos florísticos cuya forma de vida es variable, en función del sustrato y las condiciones ambientales de los sitios, las comunidades mantienen una fisonomía similar. Entre los componentes arbóreos comunes se encuentran Bursera simaruba, Ceiba aesculifolia, Cnidoscolus aconitifolius, Croton arboreus, Haematoxylum brasiletto, Hauya elegans subsp. barcenae, Leucaena esculenta, Lonchocarpus rugosus, Lysiloma divaricatum, Plumeria rubra, Pseudobombax ellipticum, Stemmadenia pubescens y Terminalia buceras. Las especies que alcanzan grandes alturas son Ficus pertusa, F. cookii, Guaiacum sanctum, Leucaena leucocephala y Lonchocharpus rugosus. Otros elementos importantes son Bonellia macrocarpa, Pilosocereus leucocephalus, Pterocereus gaumeri y Sideroxylon laetevirens. En los sitios que se encuentran próximos al BQ se observan individuos aislados en estado juvenil del género Quercus. Las formaciones de Gymnopodium antigonoides son frecuentes y abarcan grandes extensiones de terreno. Los arbustos están ausentes o se encuentran pobremente representados por Bakeridesia pittieri, Euphorbia calcarata, E. schlechtendalii y Hibiscus spiralis, que no forman un estrato definido. Dentro del estrato herbáceo, la familia Acanthaceae es la mejor representada, con 


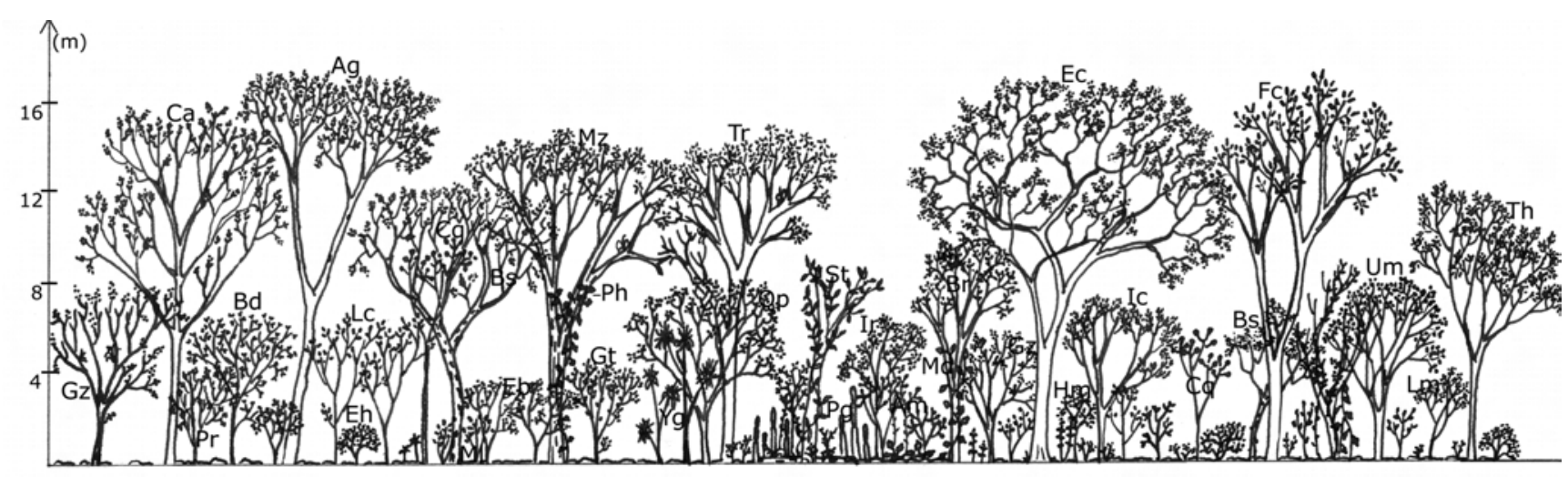

Figura 3. Esquema de vegetación de Bosque Tropical Subcaducifolio (BTSC). Ag. Astronium graveolens, Am. Acalypha macrostachya, Bd. Bauhinia divaricata, Br. Brosimum alicastrum, Bs. Bursera simaruba, Ca. Calycophyllum candidissimum, Cq. Capparidastrum quiriguense, Eb. Eugenia breedlovei, Ec. Enterolobium cyclocarpum, Eh. Erythroxylum havanense, Fc. Ficus insipida, Gt. Guatteria amplifolia, Gz. Guazuma ulmifolia, Hm. Hyperbaena mexicana, Ic. Inga chiapensis, Ir. Iresine arbuscula, Lc. Licaria caudata, Lm. Lonchocarpus minimiflorus, Md. Monstera deliciosa, Mz. Manilkara zapota, Pg. Pterocereus gaumeri, Ph. Philodendron hederaceum, Pr. Pilocarpus racemosus var. racemosus, Qp. Quadrella pringlei, Th. Trichilia hirta, Tr. Tabebuia rosea, St. Stigmaphyllon ellipticum, Um.

Ulmus ismaelis, Yg. Yucca guatemalensis.

especies como Carlowrightia arizonica, Dicliptera unguiculata, Elytraria imbricata y Ruellia inundata, entre otras. Bommeria pedata es una de las pocas especies de pteridofitas encontradas. En cuanto a epífitas, el género Tillandsia es el mejor representado en número de especies y, en ocasiones, el único. También es común encontrar especies parásitas o hemiparásitas, principalmente del género Phoradendron. Las lianas y bejucos son escasos, Hippocratea celastroides, Monstera deliciosa y Philodendron warszewiczii se encuentran como componentes perennifolios comunes de los BTC de la región (Figura 2).

Bosque tropical subcaducifolio (BTSC). Se desarrolla entre 590 y 980 m s.n.m., sobre lugares que conservan humedad, como cañadas abruptas de paredes altas en donde corren ríos intermitentes. Las condiciones topográficas de estas zonas mantienen la humedad aún en los meses más secos. En estos sitios predomina el sustrato rocoso. Por la cercanía con el BTC varios elementos florísticos se comparten. La estratificación vertical consta de uno a dos estratos: (a) arbóreo, subdividido en zona de copas (16-18 m) y zona inferior de copas $(\leq 10 \mathrm{~m})$, y (b) arbustivo $(\leq 6 \mathrm{~m})$. Dentro del primero se encuentran especies como Bursera simaruba, Ficus insipida, Manilkara zapota, Tabebuia rosea y Trichilia hirta. Entre los árboles de mayor altura (18 m) están Astronium graveolens, Calycophyllum candidissimum y Enterolobium cyclocarpum. Las especies de epífitas son pocas y su abundancia es mayor dentro de este estrato, se observan especies como Billbergia macrolepis, Brassavola cucullata, Epiphyllum sp., Guarianthe aurantiaca, Hylocereus undatus, Meiracyllium trinasutum, Prosthechea cochleata, Tillandsia caput-medusae y Trichocentrum sp. La zona inferior de copas es la más diversa, dentro de ella se encuentran Bonellia ma- crocarpa, Carpinus caroliniana, Cnidoscolus aconitifolius, Erythroxylum havanensis, Eugenia axillaris, Guarea glabra, Hyperbaena mexicana, Microtropis contracta, Myriocarpa cordifolia, Phyllanthus acuminatus, Pterocereus gaumeri, Quadrella pringlei, Sideroxylon laetevirens, Ulmus ismaelis y Yucca guatemalensis. Las lianas muestran su mejor desarrollo dentro de esta comunidad, en donde cubren la copa de los árboles bajo condiciones favorables de luz. Amphilophium paniculatum, Heteropterys laurifolia, Monstera deliciosa, Prestonia longifolia, P. grandiflora, P. mexicana y Stigmaphyllon retusum son algunas lianas comunes del BTSC. Entre los arbustos destacan Piper amalago y P. marginatum, que en algunos sitios forman un estrato continuo, aunque en algunas comunidades los arbustos son escasos. Otras especies arbustivas son Acalypha macrostachya, Ardisia escallonioides, Brahea nítida, Bunchosia lindeniana, Chamaedorea plumosa, Malpighia glabra, Neea psychotrichoides, Ouratea theophrasta y Pilocarpus racemosus var. racemosus. Las hierbas se observan como parches sobre rocas que acumulan materia orgánica, no forman un estrato. Algunas hierbas son Acalypha alopecuroidea, Adiantum capillus-veneris, A. pumilum, Anthurium schlechtendalii, Calyptocarpus wendlandii, Commicarpus scandens, Dorstenia contrajerva, Jarilla heterophylla, Onoseris onoseroides, Peperomia asarifolia, $P$. pereskifolia, Ruellia inundata, $R$. jussieuoides, Thelypteris puberula y Tradescantia zanonia (Figura 3 ).

Bosque de Quercus (BQ). El BQ se desarrolla entre $800 \mathrm{y}$ 970 m s.n.m., presenta un estrato arbóreo y uno herbáceo. Dentro de la zona se registraron dos formaciones: (a) comunidades dominadas por árboles robustos de 6-10(-12) m de altura, y (b) comunidades dominadas por árboles de 3-4 $\mathrm{m}$ de altura. La primera de ellas prospera en las laderas del 


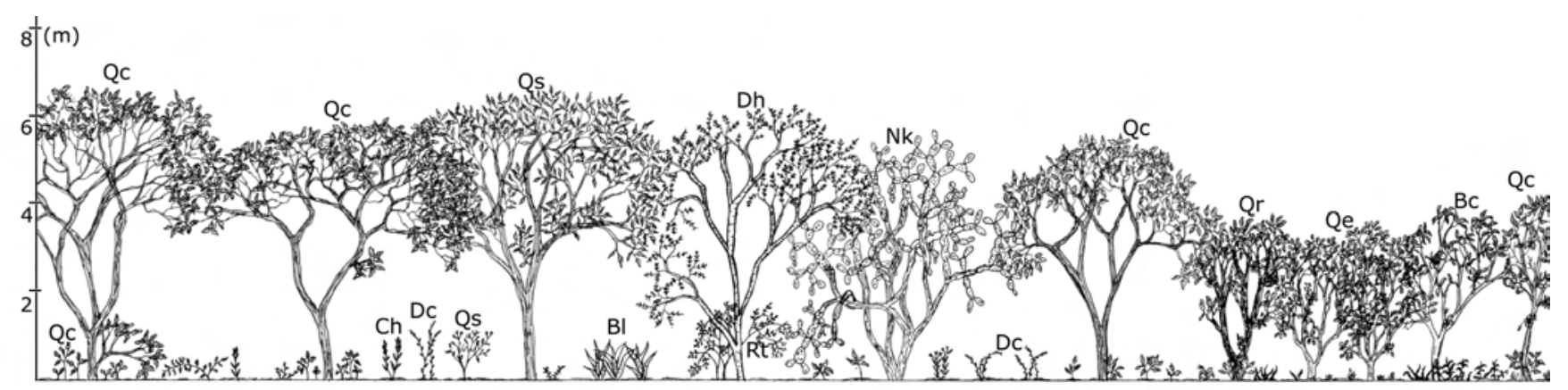

Figura 4. Bosque de Quercus (BQ), perfil de vegetación. Bc. Byrsonima crassifolia, B1. Billbergia macrolepis, Ch. Chromolaena opadoclina, Dc. Desmodium cinereum, Dh. Dahnopsis americana, Nk. Nopalea karwinskiana, Qe. Quercus elliptica, Qc. Quercus conspersa, Qr. Quercus rugosa, Qs. Quercus segoviensis, Rt. Rhus terebinthifolia.

sistema montañoso y en terrenos planos al pie de la montaña y está dominada por Quercus conspersa, Q. peduncularis y Q. segoviensis en el estrato arbóreo. El BTC y el BQ forman parte de un mismo paisaje; por ello, comparten elementos florísticos en sitios determinados. En estos sitios, el estrato arbóreo está representado por especies como Comocladia guatemalensis, Dahnopsis americana, Ficus obtusifolia, Nopalea karwinskiana, Styrax argenteus y Swietenia humilis. Los arbustos son esporádicos, entre ellos están Psidium molle y Zanthoxyllum fagara. El estrato herbáceo está compuesto por Billbergia macrolepis, Dalea versicolor var. versicolor, Gnaphalium aff. brachypterum y Triphora gentianoides. En lugares cercanos a corrientes de agua se registran especies como Ageratum corymbosum, Arthrostemma ciliatum, Bletia purpurea, Cyperus ochraceus, Euphorbia gramínea y Xanthosoma robustum. Las epífitas son escasas, especies como Tillandsia fasciculata, T. caput-medusae, Trichocentrum ascendens, se desarrollan en las ramas y troncos. Las lianas se presentan en puntos en donde el BQ comparte el espacio con elementos de BTC, donde pueden encontrarse Cissus rhombifolia, Dioscorea floribunda, Passiflora foetida y Philodendron warszewiczii. Las comunidaes dominadas por árboles de 3-4 m de altura generalmente se desarrollan en las cimas de los sistemas montañosos, en donde la neblina es constante durante el invierno. Quercus elliptica, como especie dominante, además de $Q$. peduncularis y Byrsonima crassifolia conforman el estrato arbóreo. Las epífitas son frecuentes, el género Tillandsia es el mejor representado y $T$. streptophylla es una de las especies comunes, además se encuentran Catopsis berteroniana, Guarianthe skinneri, Serpocaulon triseriale, T. caput-medusae y $T$. recurvata. El estrato herbáceo está compuesto por pocas especies en áreas con mínima perturbación, como Borreria ocymoides, Buchnera pusilla, Chromolaena opadoclinia, Lasiacis ruscifolia y Sobralia sp. La diversidad de hierbas aumenta en lugares perturbados, que albergan especies como Achimenes longiflora, Aldama dentata, Anemia hirsuta, Blechnum occidentale, Bletia roezlii, Borreria ocymoides, Bulbostylis juncoides, Cheilanthes farinosa, Chromolaena odorata, Crotalaria bupleurifolia, Cyperus aggregatus, Dryopteris patula, Eragrostis cilianensis, Habenaria macroceratitis, Pectis postrata y Zinnia peruviana. La única especie rastrera observada es Passiflora foetida (Figura 4).

\section{Discusión}

Se reportaron 642 especies, lo que equivale al $64.9 \%$ de las registradas por Reyes-García y Sousa (1997) en un inventario del BTC de la Depresión Central de Chiapas, al 8.4\% de la flora del estado de Chiapas (Breedlove, 1986), al 2.8\% de las plantas vasculares en México (Villaseñor, 2004) y al 2.6\% del total de plantas registradas para Mesoamérica (Myers et al., 2000). Si se realiza una comparación con otros estudios florísticos (Cuadro 4), se observa que la región mantiene una riqueza específica alta. En un área con menos de $21 \mathrm{~km}^{2}$ habita $60 \%$ de la cantidad total de especies registradas para el Parque Nacional Cañón del Sumidero. La diversidad encontrada es similar a la reportada por Salas-Morales et al. (2003) en el Parque Nacional Huatulco, Oaxaca y a la de Nizanda, Oaxaca (Pérez-García et al., 2001). Se observa que el número de géneros también es alto si se toma como referencia el trabajo de Reyes-García y Sousa (1997) en la Depresión Central de Chiapas. Se descartó la posibilidad de comparación a nivel de familia debido al uso de diferentes clasificaciones en los estudios.

En cuanto a número de especies, Fabaceae fue la familia mejor representada, un patrón encontrado no sólo en los bosques secos de México (Trejo, 2010), sino también a lo largo del Neotrópico (Pennington et al., 2006); así como en trabajos realizados en áreas circundantes a la zona de estudio (Gallegos-Ramos, 2009; Espinosa-Jiménez et al., 2011), en donde Fabaceae y Asteraceae fueron las familias con un mayor número de especies. Trejo (2010) comenta que para las selvas secas del Pacífico Mexicano, después de Fabaceae, Euphorbiaceae es la familia de mayor riqueza; Pennington 
Josefa ANAHI ESPINOSA-JiMÉNEZ ET AL.

Cuadro 4. Comparación de la riqueza florística de la zona de estudio con otras regiones. BTC. Bosque Tropical Caducifolio; BE. Bosque Espinoso; BTSC. Bosque Tropical Subcaducifolio; BTP. Bosque Tropical Perennifolio; BMM. Bosque Mesófilo de Montaña; BQ. Bosque de Quercus; BC. Bosque de Coníferas; P, Pastizal; VASA. Vegetación Acuática y Subacuática.

\begin{tabular}{|c|c|c|c|c|c|c|c|}
\hline Sitio & Área $\left(\mathrm{km}^{2}\right)$ & $\begin{array}{l}\text { Número } \\
\text { de familias }\end{array}$ & $\begin{array}{l}\text { Número } \\
\text { de género }\end{array}$ & $\begin{array}{l}\text { Número } \\
\text { de especies }\end{array}$ & $\begin{array}{l}\text { Altitud } \\
\text { m s.n.m. }\end{array}$ & $\begin{array}{l}\text { Tipo de } \\
\text { vegetación }\end{array}$ & Fuente \\
\hline $\begin{array}{l}\text { Distrito de } \\
\text { Tehuantepec, Oaxaca }\end{array}$ & 6,600 & 154 & 776 & 1720 & $0-1,800$ & $\begin{array}{l}\text { BTC, BE, BC, } \\
\text { VASA, BQ, P }\end{array}$ & $\begin{array}{l}\text { Torres-Colin } \\
\text { et al., } 1997\end{array}$ \\
\hline Zimatán, Oaxaca & 713 & 144 & 668 & 1348 & $0-2,580$ & $\begin{array}{l}\text { BTC, BE, BTP y BTSC, } \\
\text { BMM, BQ, BP, VASA, P }\end{array}$ & $\begin{array}{l}\text { Salas-Morales } \\
\text { et al., } 2003\end{array}$ \\
\hline $\begin{array}{l}\text { Parque Nacional } \\
\text { Huatulco, Oaxaca }\end{array}$ & 63 & 91 & 391 & 736 & $<200$ & $\begin{array}{l}\text { BTC, BE, BTSC, VASA, P, } \\
\text { Vegetación secundaria }\end{array}$ & $\begin{array}{l}\text { Salas et al., } \\
\quad 2007\end{array}$ \\
\hline Nizanda, Oaxaca & 85 & 117 & 458 & 746 & $90-500$ & BTC, VASA, P, Agrícola & $\begin{array}{l}\text { Pérez-García } \\
\text { et al., } 2001\end{array}$ \\
\hline $\begin{array}{l}\text { Depresión Central } \\
\text { de Chiapas }\end{array}$ & 9,000 & 103 & 489 & 998 & $200-1,500$ & BTC, BE & $\begin{array}{l}\text { Reyes-García y } \\
\text { Sousa, } 1997\end{array}$ \\
\hline $\begin{array}{l}\text { Parque Nacional Cañón } \\
\text { del Sumidero, Chiapas }\end{array}$ & 158.5 & 124 & 517 & 1069 & $360-1,720$ & BTC, BTSC, BQ & $\begin{array}{l}\text { Espinosa-Jiménez } \\
\text { et al., } 2011\end{array}$ \\
\hline $\begin{array}{l}\text { Cañada Juan } \\
\text { Crispín-La Chacona }\end{array}$ & 20.69 & 107 & 412 & 643 & $594-1,030$ & $\begin{array}{l}\text { BTC, BTSC, BQ, } \\
\text { Vegetación secundaria }\end{array}$ & Este trabajo \\
\hline
\end{tabular}

et al. (2006) hacen la misma aseveración para los bosques secos de México. En el presente estudio, Asteraceae ocupa el segundo lugar y Euphorbiaceae se ubica en el tercero. La riqueza de Asteraceae y la abundancia de especies herbáceas, la mayoría representantes de esta familia, se consideran como indicadores de perturbación, debido a que las asteráceas se caracterizan por su desarrollo en ambientes perturbados y por su capacidad de invadir habitats forestales (Pruski y Sancho, 2004). En cuanto a epífitas, Rzedowski (2006) comenta que, en estos tipos de vegetación, Tillandsia es el género mejor representado, lo que concuerda con los resultados de este trabajo; el centro y sur de México se consideran como centros de diversidad de Tillandsia (Benzing, 2000).

Aún cuando la zona presenta impactos graves por disturbio antropogénico, existen sitios que tienen características florísticas y estructurales de las comunidades de BTC descritas para la Depresión Central de Chiapas por Miranda (1998), información retomada y corroborada por Pennington y Sarukhán (2005), y Rzedowski (2006). Entre estas características están la representatividad del género Bursera, la presencia de cactáceas columnares y, en sitios con mayor húmedad, del género Ficus y de especies de palmas (Rzedowski, 2006; Trejo, 2010). Rzedowski (2006) señala que las epífitas son escasas dentro de los BTC, de manera que la riqueza de especies de las familias Bromeliaceae y Orchidaceae (17 y 16 sp. respectivamente), constituyen también un indicador del estado de conservación de los sitios. El endemismo que, como Lott y Atkinson (2006) mencionan, es alto a nivel de especie en los BTC del Pacífico Mexicano, pero bajo en otras jerarquías taxónomicas, constituye un atributo más que sugiere la presencia de fragmentos de bosque que valen la pena conservar.

El área de estudio presenta también heterogeneidad ambiental, característica de las selvas secas del pacífico de México (Trejo, 2010), que favorece el establecimiento de otro tipo de comunidades, como BTSC y formaciones de BQ; como consecuencia existe una alta diversidad y complejidad estructural. Entre los sitios de mayor relevancia está la La Cañada Chacona-Juan Crispin, que presenta BTSC y alberga especies típicas de ambientes húmedos dentro de una matriz de BTC, en donde se encontraron Pilocarpus racemosus var. racemosus y Ulmus ismaelis, dos nuevos registros para la flora de Chiapas. Entre las especies que aumentan el interés en la región están Triphora gentianiodes, una orquídea encontrada en BQ y considerada como una especie rara, pues aunque está reportada para parte del Norte, Centro y Sur de América, los registros de herbario son realmente pobres (MO, 2012; CAS, 2012; KEW, 2012); además de Bdallophytum oxylepis (Cytinaceae), una especie parásita de Bursera simaruba que habita en BTC y recientemente fue registrada para Chiapas (Martínez-Camilo et al., 2012).

Mesoamérica, región considerada por Myers et al. (2000) como un hotspot, es importante no sólo por su alto grado de endemismo, sino también por el alto grado de amenaza. Desafortunadamente, el área en estudio presenta la misma problemática ambiental que enfrenta la mayoría de las comunidades vegetales de esta región. Prance et al. (2000) señalan que en los trópicos la destrucción de la vegetación es más rápida que la elaboración de los inventarios. En el mismo sentido, Sosa y Dávila (1994) comentan que existen 
zonas que están transformándose rápidamente y que contienen una alta diversidad de especies que no están representadas en las colecciones; entre estas zonas incluyen al bosque de coníferas y encinos, y las selvas tropicales de la vertiente del Pacífico. A nivel local, la Depresión Central de Chiapas está considerada por Arriaga (2009) como un área con poca representatividad de RTP, debido a la falta de información respecto a su biodiversidad. En la zona en donde se ubica el sitio de estudio, la proximidad de las poblaciones humanas es la causa principal de la pérdida de grandes extensiones de cobertura vegetal que han sido y están siendo removidas por cambio de uso de suelo para la construcción de nuevos fraccionamientos habitacionales, sin que se tenga información completa de la biodiversidad. Una muestra de ello son los nuevos registros colectados, que indican que la región aún mantiene zonas con bajos índices de colección. El inventario generado en este trabajo es una útil herramienta en la toma de decisiones enfocadas a la conservación.

Si bien es cierto que el área de estudio presenta en su mayoría vegetación secundaria, también se conoce que ésta juega un papel importante en la evolución de la flora tropical (Prance, 1977), provee servicios ambientales y funciona como refugios de biodiversidad (Guariguata y Ostertag, 2002). La heterogeneidad de hábitat es también un atributo digno de conservación, es probable que las condiciones que permiten la presencia de BTC, BTSC y BQ en un área pequeña hayan dado origen a una alta riqueza florística, ya que $39.2 \%$ de las especies y $14.7 \%$ de los géneros de este estudio no se han registrado dentro del Parque Nacional Cañón del Sumidero, un área separada por tan sólo $2.6 \mathrm{~km}$ que alberga los mismos tipos de vegetación reportados en el presente trabajo; además, seis de las 13 especies endémicas a Chiapas presentes en la zona estudiada no se habían colectado para esta ANP (Espinosa-Jiménez et al., 2011).

Olson y Dinerstein (2002) comentan que la mayor diversidad de las selvas secas en el mundo ocurre en el sureste de México y en las tierras bajas de Bolivia. Ellos realizaron una propuesta para alcanzar los objetivos globales de conservación por medio de la detección a nivel mundial de eco-regiones terrestres con características únicas en biodiversidad, entre éstas se encuentran las selvas secas de México. El reto en la actualidad está en localizar áreas representantes de este ecosistema, que contengan una riqueza y endemismo distintivos y redoblar esfuerzos para evitar o mermar la pérdida de su cobertura vegetal.

\section{Agradecimientos}

Los autores agradecen de manera especial a Marco Antonio Domínguez y Yuli Beatriz López Mendoza por su valioso apoyo en la colecta y procesamiento de los ejemplares botánicos; al Dr. Christopher Davidson y Sharon Christoph por el apoyo financiero para la realización de este trabajo; a Pablo Carrillo-Reyes y a un revisor anónimo que con sus valiosas observaciones y sugerencias contribuyeron a la mejora del manuscrito.

\section{Literatura citada}

Andrade de Sousa A.G., Sousa S.M. y Martínez S.E.M. 2007. Las colecciones botánicas de Faustino Miranda en el área mesoamericana de México. En: Mancilla F.J.D. Coord. Faustino Miranda. Una Vida Dedicada a la Botánica, pp. 259-296, Instituto de Investigaciones Historicas, Universidad Michoacana de San Nicolas de Hidalgo, Fondo Editorial Morevallado, Morelia.

APG. Angiosperm Phylogeny Group. 2009. An update of the angiosperm phylogeny group classification for the ordes and families of flowering plants: APG III. Botanical Journal of the Linnean Society 161:105-121.

Arriaga C.L. 2009. Regiones prioritarias y planeación para la conservación de la biodiversidad En: Dirzo R., González R. y March I.J. Comps. Capital Natural de México, Vol. II: Estado de Conservación y Tendencias de Cambio, pp. 433-457, Comisión Nacional para el Conocimiento y Uso de la Biodiversidad, México, D.F.

Benzing D.H. 2000. Bromeliaceae: profile of an adaptative radiation. Cambridge University Press, Cambridge.

Breedlove D.E. 1986. IV Flora de Chiapas. Listados Florísticos de México. Instituto de Biología, Universidad Nacional Autónoma de México. México, D.F. CAS. California Academy of Sciences. 2012. <http://collections. calacademy.org/bot/> (consultado junio de 2012).

Ceballos G. 2007. Conservation priorities for mammals in megadiverse México: The efficiency of reserve networks. Ecological Applications 17:569-578.

Ceballos G., Cantú C. y Bezaury Creel J. 2010a. Áreas de conservación de las regiones prioritarias de las selvas secas. En: Ceballos G., Martínez L., García A., Espinoza E., Bezaury Creel J. y Dirzo R. Eds. Diversidad, Amenazas y Áreas Prioritarias para la Conservación de las Selvas Secas del Pacífico de México, pp. 349-368, Fondo de Cultura Económica, Comisión Nacional para el Conocimiento y Uso de la Biodiversidad, México, D.F.

Ceballos G., Martínez L., García A., Espinoza E. y Bezaury Creel J. 2010b. Áreas prioritarias para la conservación de las selvas secas del Pacífico mexicano. En: Ceballos G., Martínez L., García A., Espinoza E., Bezaury Creel J. y Dirzo R. Eds. Diversidad, Amenazas y Áreas Prioritarias para la Conservación de las Selvas Secas del Pacífico de México, pp. 387-392, Fondo de Cultura Económica, Comisión Nacional para el Conocimiento y Uso de la Biodiversidad, México, D.F.

CONABIO. Comisión Nacional para el Conocimiento y Uso de la Biodiversidad. 2008a. Remib. Red Mundial de Información sobre diversidad. <http://www.conabio.gob. $\mathrm{mx} / \mathrm{remib} /$ doctos/remib_esp.html> (consultado diciembre de 2011). 
CONABIO. Comisión Nacional para el Conocimiento y Uso de la Biodiversidad. 2008b. Sistema de información Biótica 5.0.104, Manual de usuario. Fideicomismo fondo para la Biodiversidad, Comisión Nacional para el Conocimiento y Uso de la Biodiversidad, México, D.F.

Crabbe J.A., Jermy A.C. y Mickel J.T. 1975. A new generis sequence for the Pteridophyte herbarium. British Fern Gazette 2:141-162.

Dirzo R. y Ceballos G. 2010. Las selvas secas de México: un reservorio de biodiversidad y laboratorio viviente. En: Ceballos G., Martínez L., García A., Espinoza E., Bezaury Creel J. y Dirzo R. Eds. Diversidad, Amenazas y Áreas Prioritarias para la Conservación de las Selvas Secas del Pacífico de México, pp. 13-17, Fondo de Cultura Económica, Comisión Nacional para el Conocimiento y Uso de la Biodiversidad, México, D.F.

Espinosa-Jiménez J.A., Pérez-Farrera M.A. y Martínez-Camilo R. 2011. Inventario florístico del Parque Nacional Cañón del Sumidero, Chiapas, México. Boletín de la Sociedad Botánica de México 89:37-82.

Gallegos-Ramos R.E. 2009. Inventario florístico de la Cañada La Chacona y parte occidente del Parque Nacional Cañón del Sumidero, Chiapas, México. Tesis de Licenciatura. Universidad de Ciencias y Artes de Chiapas. Tuxtla Gutiérrez, Chiapas, México. 88 pp.

González-Espinosa M., Ramírez-Marcial N., MéndezDewar G., Galindo-Jaimes L. y Golicher D. 2005 Riqueza de especies de árboles en Chiapas: variación espacial y dimensiones ambientales asociadas al nivel regional. En: González-Espinosa M., Ramírez-Marcial N. y Ruiz-Montoya L. Eds. Diversidad Biológica en Chiapas, pp. 81-125, El Colegio de la Frontera Sur, COCyTECH, Plaza y Valdes Editores, México, D.F.

Gordon J.E., Bowen-Jones E. y González M.A. 2006. What determines dry forest conservation in Mesoamerica? Opportunism and pragmatism in Mexican and Nicaraguan protected areas. En: Pennington R.T., Lewis G.P. y Ratter J.A. Eds. Neotropical Savannas and Seasonally Dry Forest: Plant Diversity, Biogeography, and Conservation, pp. 343-358, The Systematics Association, CRC Press, Taylor \& Francis Group, Boca Raton.

Guariguata M.R. y Ostertag R. 2002. Sucesión secundaria. En: Guariguata M.R. y Kattan G.H. Comps. Ecología y Conservación de Bosques Neotropicales, pp.591-623, Libro Universitario Regional, Cartago.

INEGI. Instituto Nacional de Estadística, Geografía e Informática. 1985a. Carta Edafológica 1:250 000 Tuxtla Gutiérrez E15-11, Instituto Nacional de Estadística, Geografía e Informática, México, D.F.

INEGI. Instituto Nacional de Estadística, Geografía e Informática. 1985b. Carta Geológica 1:250 000 Tuxtla Gutiérrez E15-11, Instituto Nacional de Estadística, Geografía e Informática, México, D.F.

IUCN. International Union for Conservation of Nature. 2010.
IUCN Red List of Threatened Species. Versión 2010.4. <www.iucnredlist.org> (consultado agosto de 2010).

KEW. Royal Botanic Gardens. 2012. <http://apps.kew.org/ herbcat/gotoHomePage.do> (consultado diciembre de 2011).

Lot A. y Chiang F. 1986. Manual de Herbario. Administración y Manejo de Colecciones, Técnicas de Recolección y Preparación de Ejemplares Botánicos. Consejo Nacional de la Flora de México. A.C., México, D.F.

Lott E.J. y Atkinson T.H. 2006. Mexican and Central American seasonally dry tropical forests: Chamela-Cuixmala, Jalisco, as a focal point for comparison. En: Pennington R.T., Lewis G.P. y Ratter J.A. Eds. Neotropical Savannas and Seasonally Dry Forests: Plant Diversity, Biogeography, and Conservation, pp. 315-342, The Systematics Association, CRC Press, Taylor \& Francis Group, Boca Raton.

Lott E.J. y Atkinson T.H. 2010. Diversidad florística. En: Ceballos G., Martínez L., García A., Espinoza E., Bezaury Creel J. y Dirzo R. Eds. Diversidad, Amenazas y Áreas Prioritarias para la Conservación de las Selvas Secas del Pacífico de México, pp. 63-76, Fondo de Cultura Económica, Comisión Nacional para el conocimiento y Uso de la Biodiversidad, México, D.F.

Martínez-Camilo R., Martínez-Meléndez N. y Pérez-Farrera M.A. 2012. Nuevo registro de Bdallophytum oxylepis (Malvales: Cytinaceae) para Chiapas. Revista Mexicana de Biodiversidad 83:1230-1232.

Mickel J.T. y Smith A.R. 2004. The pteridophytes of Mexico. Memoirs of the New York Botanical Garden 88:11054.

Miranda F. 1998. La Vegetación de Chiapas. $3^{\mathrm{a}}$ ed. Consejo estatal para la Cultura y las Artes de Chiapas, Tuxtla Gutiérrez.

Miranda F. y Hernández X.E. 1963. Los tipos de vegetación de México y su clasificación. Boletín de la Sociedad Botánica de México 28:29-179.

MO. Missouri Botanical Garden. 2012. Flora Mesoamericana. <http://www.tropicos.org/Project/FM> (consultado enero de 2012).

Myers N., Mittermeier R.A., Mittermeier C.G., da Fonseca G.A.B. y Kent J. 2000. Biodiversity hotspots for conservation priorities. Nature 403:853-858.

Olson D.M. y Dinerstein E. 2002. The Global 200: priority ecoregions for global conservation. Annals of the Missouri Botanical Garden 89:199-224.

Pennington T.D. y Sarukhán J. 2005. Árboles Tropicales de México: Manual para la Identificación de las Principales Especies. 3a ed. Universidad Nacional Autónoma de México, Fondo de Cultura Económica. México, D.F.

Pennington R.T., Lewis G.P. y Ratter J.A. 2006. An overview of the plant diversity, biogeography and conservation of neotropical savannas and seasonally dry forests. En: Pennington R.T., Lewis G.P. y Ratter J.A. Eds. Neotropical Savannas and Seasonally Dry forest: Plant Di- 
versity, Biogeography, and Conservation, pp. 1-25, The Systematics Association, CRC Press, Taylor \& Francis Group, Boca Raton.

Pérez-García E.A., Meave J. y Gallardo C. 2001. Vegetación y flora de la región de Nizanda, Istmo de Tehuantepec, Oaxaca, México. Acta Botanica Mexicana 56:19-88.

Prance G.T. 1977. Floristic inventory of the tropics: where do we stand? Annals of the Missouri Botanical Garden 64:659-684.

Prance G.T., Beentje H., Dransfield J. y Johns R. 2000. The tropical flora remains undercollected. Annals of Missouri Botanical Garden 87:67-71.

Pruski J.F. y Sancho G. 2004. Asteraceae. En: Smith N., Mori S.A., Henderson A., Stevenson D.W. y Heald S.V. Eds. Flowering Plants of the Neotropics, pp. 33-39, Princeton University Press, Princeton.

Reyes-García A. y Sousa S.M. 1997. Depresión Central de Chiapas. La Selva Baja Caducifolia. XVII. Listados Florísticos de México. Instituto de Biología, Universidad Nacional Autónoma de México. México, D.F.

Rzedowski J. 2006. Vegetación de México. 1ra edición digital. Comisión Nacional para el Conocimiento y Uso de la Biodiversidad, México, D.F.

Salas-Morales S.H., Saynes-Vásquez A. y Schibli L. 2003. Flora de la costa de Oaxaca, México: Lista florística de la región de Zimatán. Boletín de la Sociedad Botánica de México 72:21- 58.

SEMARNAT. Secretaría del Medio Ambiente y Recursos Naturales. 2010. Norma Oficial Mexicana NOM-059SEMARNAT-2010. Protección ambiental-Especies nativas de México de Flora y Fauna Silvestres-Categorías de
Riesgo y especificaciones para su inclusión, exclusión o cambio--Lista de Especies en Riesgo.

Sosa V. y Dávila P. 1994. Una evaluación del conocimiento florístico de México. Annals of the Missouri Botanical Garden 81:749-757.

Toledo V.M. y Ordóñez M.J. 1998. El panorama de la biodiversidad de México: una revisión de los hábitats terrestres. En: Ramamoorthy T.P., Bye R, Lot A. y Fa J. Comps. Diversidad Biológica de México, Orígenes y Distribución, pp. 739-757, Instituto de Biología, Universidad Nacional Autónoma de México, México, D.F.

Torres-Colín, R., L. Torres C., P. Dávila A. y J.L. Villaseñor R. 1997. Flora del Distrito de Tehuantepec, Oaxaca. Listados Florísticos de México XVI. Instituto de Biología, Universidad Nacional Autónoma de México, México, D.F.

Trejo I. 2010. Las selvas secas del Pacífico Mexicano. En: Ceballos G., Martínez L., García A., Espinoza E., Bezaury Creel J. y Dirzo R. Eds. Diversidad, Amenazas y Áreas Prioritarias para la Conservación de las Selvas Secas del Pacífico de México, pp. 41-51, Fondo de Cultura Económica, Comisión Nacional para el Conocimiento y Uso de la Biodiversidad, México, D.F.

Vargas M.F., Escobar S. y del Ángel R. Comps. 2000. Áreas Naturales Protegidas de México con Decretos Federales. Instituto Nacional de Ecología, Secretaría de Medio Ambiente, Recursos Naturales y Pesca, Red para el Desarrollo Sostenible, A. C., Programa de Naciones Unidas para el Desarrollo, México, D.F.

Villaseñor J.L. 2004. Los géneros de plantas vasculares de la flora de México. Boletín de la Sociedad Botánica de México 75:105-135.

Recibido: 23 de enero de 2013

Aceptado: 30 de mayo de 2013 
Apéndice 1. Lista florística de la Cañada La Chacona-Juan Crispín y zonas adyacentes, Chiapas, México. Tipo de Vegetación: BTC = Bosque tropical caducifolio, BTC (VS) = Bosque tropical caducifolio (vegetación secundaria), BTSC = Bosque tropical subcaducifolio, BTSC (VS) = Bosque tropical subcaducifolio (vegetación secundaria), BQ = Bosque de Quercus, BQ (VS) = Bosque de Quercus (vegetación secundaria). Fenología: FI = Flor, Fr = Fruto, Fe = Fértil (helechos), In = Infértil. Mes de colecta: $\mathbf{1}=$ Enero hasta $\mathbf{1 2}$ = Diciembre. Forma de crecimiento: Á = Árbol, Ar = Arborescente, $\mathbf{A b}=$ Arbusto, $\mathbf{C l}=\mathrm{Columnar}, \mathbf{H i}=\mathbf{H i e r b a}$, $\mathbf{L i}=$ Liana o bejuco. Distribución: Cam = Campeche, $\mathbf{C h}=$ Chiapas, $\mathbf{G}=$ Guatemala, $\mathbf{O}=$ Oaxaca, $\mathbf{V}=$ Veracruz, $\mathbf{Y}=$ Yucatán. Estatus de conservación: NOM: A = Amenazada, $\mathbf{P}=$ En peligro de extinción, $\mathbf{P r}=$ Sujeta a protección especial IUCN: $\mathbf{E N}=$ En peligro, $\mathbf{L C}=$ Preocupación menor, $\mathbf{L R} / \mathbf{n t}=$ Bajo riesgo/casi amenazada, $\mathbf{V U}=$ Vulnerable. $\mathbf{N d}=$ datos no disponibles.

\begin{tabular}{llllll}
\hline Clado/Familia/Especie/ & Colector & FC & FN & MC & TV \\
\hline PTERIDOPHYTA & & & &
\end{tabular}

\section{Athyriaceae}

Diplazium franconis Liebm.

Hartmann R. 5 (HEM)

A. López C. 1312 (HEM)

A. López C. 1395 (HEM)

Blechnum polypodioides Raddi

Dryoptyeridaceae

Dryopteris karwinskyana (Mett.) Kuntze

Dryopteris patula (Sw.) Underw

\section{Polypodiaceae}

Polypodium furfuraceum Schltdl. \& Cham.

Serpocaulon triseriale (Sw.) A.R.Sm.

\section{Pteridaceae}

Adiantum andicola Liebm.

Adiantum braunii Mett. ex Kuhn

Adiantum capillus-veneris L.

Adiantum concinnum Humb.

\& Bonpl. ex Willd

Adiantum pulverulentum L.

Adiantum pumilum Sw.

Adiantum tricholepis Fée

Bommeria pedata (Sw.) E.Fourn.

Cheilanthes farinosa (Forssk.) Kaulf.

Cheilanthes pyramidalis Fée

Cheiloplecton rigidum (Sw.) Fée

Cheiloplecton rigidum (Sw.) Fée var rigidum

Pteris longifolia L.

\author{
A. López C. 1411 (HEM) \\ A. López C. 1317 (HEM)
}

A. López C. 1265 (HEM)

A. López C. 1402 (HEM),

E. Palacios E. 1281(CHIP)

M. A. Dominguez V.239 (HEM)

J. Lazaro Z. 2 (HEM), Martínez

O. M.2 (HEM)

A. López C. 1272 (HEM)

M. A. Dominguez V.85, 237 (HEM)

Hartmann R.4 (HEM)

M. A. Domínguez V. 643 (HEM)

A. Gómez V.7 (HEM)

A. López C. 1232, 1314 (HEM)

A. López C. 1185, 1306 (HEM),

I. Serrano S. 13 (HEM)

A. López C. 1315 (HEM)

I. Serrano S. 5 (HEM), S. K.

Galdámez E. 5 (HEM)

E. Palacios E.1692 (CHIP)

A. Gómez V. 15 (HEM), J. Lazaro

Z. 15 (HEM), Martínez O. M.

15 (HEM)

A. López C. 1313 (HEM)

Angelita López Cruz 1363 (HEM)

$\mathrm{Hi}$

$\mathrm{Hi}$

$\mathrm{Hi}$

M. A. Domínguez V. 87 (HEM)

$\mathrm{Hi}$

$\mathrm{Hi}$

$\mathrm{Hi}$

$\mathrm{Hi}$

$\mathrm{Hi}$

$\mathrm{Hi}$

$\mathrm{Hi}$

$\mathrm{Hi}$

$\mathrm{Hi}$

$\mathrm{Hi}$

$\mathrm{Hi}$

$\mathrm{Hi}$

$\mathrm{Hi}$
$\mathrm{Fe}$

4

BTSC

$\begin{array}{llll}\mathrm{Hi} & \mathrm{Fe} & 8 & \mathrm{BQ} \\ \mathrm{Hi} & \mathrm{Fe} & 9 & \mathrm{BQ}(\mathrm{VS})\end{array}$

$\mathrm{Hi}$

$\mathrm{Hi}$.

Fe

$\mathrm{Fe}$

9

$\mathrm{BQ}(\mathrm{VS})$

$\mathrm{BQ}$

$\mathrm{Hi}$

$\mathrm{Hi}$

$$
\mathrm{Fe}
$$

$\mathrm{Fe}$

7

9

BTC

$\mathrm{BQ}$

\section{Schizaeaceae}

Anemia oblongifolia (Cav.) Sw.

\section{Thelypteridaceae}

Thelypteris puberula (Baker) C.V.Morton

Fe

$\mathrm{Fe}$

3

10

BQ

$\mathrm{Fe}$

$\mathrm{Fe}$

7

2,3

BTSC

Fe

Fe

$\mathrm{Fe}$

$\mathrm{Fe}$

$\mathrm{Fe}$

\section{4}

$$
2
$$

10

$$
8
$$

3, 8, 10

BTC (VS), BQ

\section{Hi}

$$
\mathrm{Fe}
$$

Fe

$$
8
$$$$
10
$$

BQ

the

$\mathrm{Nd}$

$\mathrm{Fe}$

10

BTC

BTC

BTSC

BTSC

BTSC

$B Q, B T S C$ 


\begin{tabular}{|c|c|c|c|c|c|}
\hline Clado/Familia/Especie/ & Colector & FC & FN & MC & TV \\
\hline \multicolumn{6}{|l|}{ GIMNOSPERMAE } \\
\hline \multicolumn{6}{|l|}{ Cupressaceae } \\
\hline Taxodium mucronatum Ten. & registro observado $s / n$ & Á & In & 4 & BTSC (VS) \\
\hline \multicolumn{6}{|l|}{ ANGIOSPERMAE } \\
\hline \multicolumn{6}{|l|}{ MAGNÓLIDAS } \\
\hline \multicolumn{6}{|l|}{ Annonaceae } \\
\hline Annona purpurea Moc. \& Sessé ex Dunal & M. A. Domínguez V. 401 (HEM) & Á & $\mathrm{Fl}$ & 5 & BTC (VS) \\
\hline Annona reticulata $\mathrm{L}$. & M. A. Domínguez V. 262 (HEM) & Á & $\mathrm{Fr}$ & 3 & BTC (VS) \\
\hline Guatteria amplifolia Triana \& Planch. & $\begin{array}{l}\text { A. López C. 1287, } 1291 \text { (HEM), } \\
\text { M. A. Domínguez V. 81, 109, 538, } \\
599 \text { (HEM) }\end{array}$ & Á, $\mathrm{Ab}$ & $\mathrm{Fl}, \mathrm{Fr}$ & 2,7 & $\begin{array}{l}\text { BTSC, BTC } \\
\text { (VS), BTC, }\end{array}$ \\
\hline Malmea depressa (Baill.) R.E.Fr. & E. Palacios E.1254, 1288 (CHIP) & Á & $\mathrm{Nd}$ & $\mathrm{Nd}$ & BTC \\
\hline Sapranthus campechianus (Kunth) Standl. & $\begin{array}{l}\text { A. López C. } 1428 \text { (HEM), M. A. } \\
\text { Domínguez V. } 174 \text { (HEM) }\end{array}$ & $\mathrm{Ab}, \mathrm{A}$ & $\mathrm{Fr}, \mathrm{Fl}$ & 3,6 & BTC (VS) \\
\hline \multicolumn{6}{|l|}{ Hernandiaceae } \\
\hline Gyrocarpus americanus Jacq. & $\begin{array}{l}\text { D. E. Breedlove } 30363 \text { (CAS), } \\
\text { M. A. Domínguez V. 32, } 71 \text { (HEM) }\end{array}$ & Á & $\mathrm{Fr}$ & $1,2,12$ & BTC, BTSC \\
\hline \multicolumn{6}{|l|}{ Lauraceae } \\
\hline Licaria caudata (Lundell) Kosterm. & $\begin{array}{l}\text { A. López C. } 1199 \text { (HEM), M. A. } \\
\text { Domínguez V. } 79 \text { (HEM), } \\
\text { V. Barbosa } 25 \text { (HEM) BTC (VS) }\end{array}$ & Á & $\mathrm{Fr}$ & $2,3,9$ & BTSC, BTSC \\
\hline Licaria coriacea (Lundell) Kosterm. & $\begin{array}{l}\text { E. Palacios E. \& T. Cabrera } \\
\text { C. } 2404 \text { (CHIP),. }\end{array}$ & Á & $\mathrm{Nd}$ & $\mathrm{Nd}$ & $\mathrm{Nd}$ \\
\hline Nectandra coriacea (Sw.) Griseb. & M. A. Domínguez V. 533 (HEM) & Á & $\mathrm{Fl}$ & 7 & BTC (VS) \\
\hline Nectandra salicifolia (Kunth) Nees & A. López C. 1088 (HEM), BTC (VS) & Á & $\mathrm{Fr}$ & 2 & $\mathrm{Nd}$ \\
\hline \multicolumn{6}{|l|}{ Piperaceae } \\
\hline Peperomia asarifolia Schltdl. \& Cham & $\begin{array}{l}\text { A. López C. } 1268 \text { (HEM), E. } \\
\text { Palacios E. 1278, } 2439 \text { (CHIP), } \\
\text { M. A. Domínguez V. } 621 \text { (HEM), } \\
\text { O. Farrera S. \&C. Méndez } 4249 \\
\text { (CHIP) }\end{array}$ & $\mathrm{Hi}$ & $\mathrm{Fl}$ & 7,8 & $\mathrm{BQ}, \mathrm{BTSC}$ \\
\hline Peperomia pereskiifolia (Jacq.) Kunth & $\begin{array}{l}\text { M. A. Domínguez V. } 90 \text { (HEM), } \\
\text { P. Domínguez V. } 24 \text { (HEM) }\end{array}$ & $\mathrm{Hi}$ & $\mathrm{Fr}$ & 2,9 & BTC, BTSC \\
\hline Piper amalago L. & $\begin{array}{l}\text { A. López C. 1084, 1238, 1271, } \\
\text { 1279, } 1284 \text { (HEM), López G. } \\
\text { 5(HEM), Maldonado A.13-bis } \\
\text { (HEM), M. A. Domínguez V. } \\
539 \text { (HEM) }\end{array}$ & $\mathrm{Ab}$ & $\mathrm{Fl}, \mathrm{Fr}$ & $2,7,9$ & $\begin{array}{l}\text { BTC, BTC }(V S), \\
\text { BTSC, BTSC }\end{array}$ \\
\hline Piper marginatum Jacq. & $\begin{array}{l}\text { A. López C. } 1285 \text { (HEM), García } \\
\text { J. } 4 \text { (HEM), M. A. Domínguez V. } \\
623 \text { (HEM) }\end{array}$ & $\mathrm{Ab}$ & $\mathrm{Fl}$ & $7,8,9$ & BQ, BTSC \\
\hline \multicolumn{6}{|l|}{ Monocotiledóneas } \\
\hline \multicolumn{6}{|l|}{ Asparagaceae } \\
\hline Agave angustifolia Haw. & F. Miranda G. 6491bis (MEXU) & $\mathrm{Hi}$ & $\mathrm{Nd}$ & 8 & BTC \\
\hline Agave grijalvensis Ullrich Ch & M. A. Domínguez V. 36, 181 (HEM) & $\mathrm{Hi}$ & $\mathrm{Fl}$ & 1,3 & BTC $\operatorname{Pr}$ \\
\hline
\end{tabular}




$\frac{\text { Clado/Familia/Especie/ }}{\text { Manfreda scabra (Ortega) McVaugh }}$

Yucca guatemalensis Baker

\section{Alstroemeriaceae}

Bomarea edulis (Tussac) Herb.

\section{Amaryllidaceae}

Pancratium littorale Jacq.

\section{Araceae}

Anthurium schlechtendalii Kunth

Anthurium schlechtendalii Kunth subsp. Schlechtendalii

Monstera deliciosa Liebm.

Philodendron hederaceum (Jacq.) Schott

Philodendron warszewiczii K.Koch \&

C.D.Bouché

Syngonium neglectum Schott

Syngonium podophyllum Schott

Xanthosoma robustum Schott

\section{Arecaceae}

Brahea nitida André

Chamaedorea plumosa Hodel

\section{Bromeliaceae}

Billbergia macrolepis L.B.Sm.

Bromelia hemispherica Lam.

Bromelia karatas L.

Catopsis berteroniana (Schult. \& Schult. f.) Mez

Hechtia glomerata Zucc.

Pitcairnia breedlovei L.B. Sm. Ch Pitcairnia chiapensis Miranda Ch Tillandsia achyrostachys C.J.Morren Tillandsia aff. balbisiana Schult. $\mathrm{f}$.

Tillandsia caput-medusae E.Morren

$\begin{array}{lllll}\text { Colector } & \text { FC } & \text { FN } & \text { MC } & \text { TV } \\ \begin{array}{l}\text { E. Palacios E. \& D. E. Breedlove } \\ 751(\text { CHIP })\end{array} & \text { Hi } & \text { Nd } & \mathrm{Nd} & \mathrm{Nd} \\ \begin{array}{l}\text { M. A. Domínguez V. } 653(\mathrm{HEM}) \\ \text { A }\end{array} & \text { in } & 3 & \text { BTSC }\end{array}$

\begin{tabular}{|c|c|c|c|}
\hline $\begin{array}{l}\text { A. López C. 1104,1178 (HEM), } \\
\text { E. Tápia G. s/n (HEM), O. Farrera }\end{array}$ & $\mathrm{Li}$ & $\mathrm{Fr}$ & $2,3,9$ \\
\hline
\end{tabular}

S. \& C. Méndez 42565 (CHIP)

E. Palacios E.1230 (CHIP)

$\mathrm{Hi} \quad \mathrm{Nd} \quad \mathrm{Nd} \quad \mathrm{Nd}$

\begin{tabular}{|c|c|c|c|c|}
\hline $\begin{array}{l}\text { A. López C. } 1151 \text { (HEM), M. A. } \\
\text { Domínguez V. 61, } 102 \text { (HEM) }\end{array}$ & $\mathrm{Hi}$ & $\mathrm{Fl}$ & 2,3 & $\begin{array}{l}\text { BQ, BTC, } \\
\text { BTSC }\end{array}$ \\
\hline $\begin{array}{l}\text { Ballinas S. } 12 \text { (HEM), M. Palacios } \\
\text { Ríos } 2675 \text { (UAMIZ) }\end{array}$ & $\mathrm{Hi}$ & $\mathrm{Nd}$ & 9,8 & BTC \\
\hline A. López C. 1204 (HEM) & $\mathrm{Li}$ & $\mathrm{Fr}$ & 3 & BTC (VS) \\
\hline M. A. Domínguez V. 639 (HEM) & $\mathrm{Li}$ & $\mathrm{Fr}$ & 8 & $\mathrm{BQ}$ \\
\hline $\begin{array}{l}\text { D. E. Breedlove } 69987 \text { (CAS), } \\
\text { M. A. Domínguez V. } 389 \text { (HEM) }\end{array}$ & $\mathrm{Hi}$ & $\mathrm{Fr}$ & 4 & BTC \\
\hline A. López C. 1258 (HEM) & $\mathrm{Li}$ & $\mathrm{Fr}$ & 7 & BTC (VS), \\
\hline $\begin{array}{l}\text { A. López C. } 1251 \text { (HEM), Faustino } \\
\text { Miranda González5122 (MEXU) }\end{array}$ & $\mathrm{Li}$ & $\mathrm{Fl}$ & 1,7 & BTC (VS) \\
\hline M. A. Domínguez V. 647 (HEM) & $\mathrm{Hi}$ & in & 3 & $B Q$ \\
\hline
\end{tabular}

registro observado $s / n$

$\mathrm{Ar}$

A. López C. 1194 (HEM), M. A. Ar Domínguez V. 602 (HEM)

E. Palacios E.2359 (CHIP), M. A. Domínguez V. 437, 646 (HEM)

A. López C. 1213, 1125 (HEM),

M. A. Domínguez V. 563 (HEM)

E. Palacios E.1221 (CAS, CHIP),

E. Palacios E.1693 (CHIP)

A. López C. 1355 (HEM),

M. A. Domínguez V. 2, 29, 123, Hi 180 (HEM)

M. A. Domínguez V. 38 (HEM) Hi

M. A. Domínguez V. 12 (HEM) Hi

M. A. Domínguez V. 428 (HEM)

A. López C. 1356 (HEM), M. A.

Domínguez V. 228 (HEM)

A. López C. 1404, 1406, $1413 \quad \mathrm{Hi}$

(HEM), M. A. Domínguez V. 110,

187, 226, 230, 243, 429, 531 (HEM)

$\begin{array}{llll}\mathrm{Hi} & \mathrm{Fl} & 3,5 & \text { BQ, BTC } \\ \mathrm{Hi} & \mathrm{Fl}, \mathrm{Fr} & 2,4,7 & \text { BTC, BTC (VS) } \\ \mathrm{Hi} & \mathrm{Nd} & \mathrm{Nd} & \text { BTC } \\ \mathrm{Hi} & \mathrm{Fr} & 9 & \text { BQ. Pr } \\ \mathrm{Hi} & \mathrm{Fl}, \mathrm{Fr} & 1,2,3 & \text { BTC } \\ \mathrm{Hi} & \mathrm{Fl} & 2 & \text { BTC } \\ \mathrm{Hi} & \mathrm{In} & 1 & \text { BTC } \\ \mathrm{Hi} & \mathrm{Fl} & 5 & \text { BTC } \\ \mathrm{Hi} & \mathrm{Fl}, \mathrm{Fr} & 3,9 & \text { BQ } \\ \mathrm{Hi} & \mathrm{Fl}, \mathrm{Fr} & 2,3,5, & \text { BQ, BTC, BTC } \\ & & 7,9 & \text { (VS), BTSC }\end{array}$




\begin{tabular}{|c|c|c|c|c|c|}
\hline Clado/Familia/Especie/ & Colector & FC & FN & MC & TV \\
\hline Tillandsia fasciculata Sw. & $\begin{array}{l}\text { A. López C. } 1116 \text { (HEM), M. A. } \\
\text { Domínguez V. 250, } 626 \text { (HEM) }\end{array}$ & $\mathrm{Hi}$ & $\mathrm{Fl}, \mathrm{Fr}$ & $2,3,8$ & BQ, BTC \\
\hline Tillandsia juncea (Ruiz \& Pav.) Poir. & A. López C. 1156, 1252 (HEM) & $\mathrm{Hi}$ & $\mathrm{Fl}$ & 3,7 & $\mathrm{BQ}, \mathrm{BTC}(\mathrm{VS})$ \\
\hline Tillandsia limbata Schltdl & H. B. Frederick 1633 (MEXU) & $\mathrm{Hi}$ & $\mathrm{Nd}$ & 6 & BTC \\
\hline Tillandsia makoyana Baker & $\begin{array}{l}\text { A. López C. } 1108 \text { b, } 1294 \text { (HEM), } \\
\text { M. A. Domínguez V. } 658 \text { (HEM) }\end{array}$ & $\mathrm{Hi}$ & $\mathrm{Fr}$ & $2,7,8$ & $\mathrm{BQ}, \mathrm{BTC}(\mathrm{VS})$, \\
\hline Tillandsia polystachia (L.) L. & $\begin{array}{l}\text { E. Romero B. } 23 \text { (HEM), M. A. } \\
\text { Domínguez V. } 152 \text { (HEM) }\end{array}$ & $\mathrm{Hi}$ & $\mathrm{Fr}$ & 2,10 & $\begin{array}{l}\text { BTSC, BTC } \\
\text { (VS) }\end{array}$ \\
\hline Tillandsia recurvata (L.) L. & $\begin{array}{l}\text { A. López C. } 1401 \text { (HEM), M. A. } \\
\text { Domínguez V. 165, 220, } 528 \text { (HEM), }\end{array}$ & $\mathrm{Hi}$ & $\mathrm{Fr}$ & $2,3,7,9$ & $\mathrm{BQ}, \mathrm{BTC}(\mathrm{VS})$ \\
\hline Tillandsia schiedeana Steud. & $\begin{array}{l}\text { M. A. Domínguez V. 3, 19, 51, } \\
\text { 111, } 446 \text { (HEM) }\end{array}$ & $\mathrm{Hi}$ & $\mathrm{Fl}, \mathrm{Fr}$ & $1,2,6$ & $\begin{array}{l}\text { BTC, BTC } \\
\text { (VS), BTSC }\end{array}$ \\
\hline $\begin{array}{l}\text { Tillandsia schiedeana Steud. } \\
\text { subsp. schiedeana }\end{array}$ & H. B. Frederick 1640 (MEXU) & $\mathrm{Hi}$ & $\mathrm{Nd}$ & 6 & BTC \\
\hline $\begin{array}{l}\text { Tillandsia streptophylla Scheidw. } \\
\text { ex C.Morren }\end{array}$ & $\begin{array}{l}\text { A. López C. 1168, } 1354 \text { (HEM), } \\
\text { M. A. Domínguez V. } 227 \text { (HEM), }\end{array}$ & $\mathrm{Hi}$ & $\mathrm{Fl}, \mathrm{Fr}$ & 3,9 & $\mathrm{BQ}$ \\
\hline \multicolumn{6}{|l|}{ Commelinaceae } \\
\hline $\begin{array}{l}\text { Callisia gentlei var. macdougallii } \\
\text { (Miranda) D.R.Hunt Ch }\end{array}$ & $\begin{array}{l}\text { O. Farrera S \& C. Méndez } \\
4251 \text { (CHIP). }\end{array}$ & $\mathrm{Hi}$ & $\mathrm{Nd}$ & $\mathrm{Nd}$ & BTSC \\
\hline Commelina erecta $\mathrm{L}$. & M. A. Domínguez V. 493 (HEM) & $\mathrm{Hi}$ & $\mathrm{Fl}$ & 6 & BTC (VS) \\
\hline Tradescantia zanonia (L.) Sw. & M. A. Domínguez V. 94 (HEM) & $\mathrm{Hi}$ & $\mathrm{Fr}$ & 2 & BTSC \\
\hline \multicolumn{6}{|l|}{ Cyperaceae } \\
\hline Bulbostylis juncoides (Vahl) Kük. ex Osten & A. López C. 1341, 1365 (HEM) & $\mathrm{Hi}$ & $\mathrm{Fl}$ & 9 & BQ \\
\hline Bulbostylis vestita (Kunth) C.B.Clarke & A. López C. 1327 (HEM) & $\mathrm{Hi}$ & $\mathrm{Fr}$ & 8 & BQ \\
\hline Cyperus aggregatus (Willd.) Endl. & A. López C. 1360 (HEM) & $\mathrm{Hi}$ & $\mathrm{Fl}$ & 9 & $\mathrm{BQ}$ \\
\hline Cyperus amabilis Vahl & A. López C. 1342 (HEM) & $\mathrm{Hi}$ & $\mathrm{Fr}$ & 9 & $\mathrm{BQ}$ \\
\hline Cyperus cuspidatus Kunth & D. E. Breedlove 69909 (DS) & $\mathrm{Hi}$ & $\mathrm{Nd}$ & 9 & BTSC \\
\hline Cyperus ochraceus Vahl & M. A. Domínguez V. 244 (HEM) & $\mathrm{Hi}$ & $\mathrm{FI}$ & 3 & $B Q$ \\
\hline Pycreus polystachyos (Rottb.) P.Beauv. & A. López C. 1362 (HEM) & $\mathrm{Hi}$ & $\mathrm{Fl}$ & 9 & BQ \\
\hline Scleria lithosperma (L.) Sw & $\begin{array}{l}\text { R. Gallegos R. \& F. Najarro } 225 \\
\text { (CHIP) }\end{array}$ & $\mathrm{Hi}$ & $\mathrm{Nd}$ & $\mathrm{Nd}$ & BTC \\
\hline \multicolumn{6}{|l|}{ Dioscoreaceae } \\
\hline $\begin{array}{l}\text { Dioscorea carpomaculata O.Téllez \& } \\
\text { B.G.Schub. }\end{array}$ & A. López C. 1399 (HEM) & $\mathrm{Li}$ & $\mathrm{Fl}$ & 9 & BQ \\
\hline $\begin{array}{l}\text { Dioscorea carpomaculata O.Téllez \& } \\
\text { B.G.Schub. var. carpomaculata }\end{array}$ & F. Miranda G. $6417 b$ & $\mathrm{Li}$ & $\mathrm{Nd}$ & 6 & $\mathrm{Nd}$ \\
\hline Dioscorea cyanisticta Donn.Sm. & A. López C. 1090 (HEM) & $\mathrm{Li}$ & $\mathrm{Fr}$ & 2 & BTC (VS), \\
\hline Dioscorea floribunda M.Martens \& Galeotti & $\begin{array}{l}\text { D. E. Breedlove } 69919 \text { (CAS), F. } \\
\text { Miranda G. } 6461 \text { (CHIP), } 7258 \\
\text { (MEXU), M. A. Domínguez V. 167, } \\
\text { 321, 555, } 628 \text { (HEM) }\end{array}$ & $\mathrm{Li}$ & $\mathrm{Fl}, \mathrm{Fr}$ & $\begin{array}{l}2,3 \\
7,8\end{array}$ & $\begin{array}{l}\text { BQ, BTC } \\
\text { (VS), BTSC }\end{array}$ \\
\hline $\begin{array}{l}\text { Dioscorea mesoamericana O.Téllez } \\
\text { \& A.I.Martínez Ch, O }\end{array}$ & E. Palacios E. 1696 (CHIP) & $\mathrm{Li}$ & $\mathrm{Nd}$ & $\mathrm{Nd}$ & BTC \\
\hline $\begin{array}{l}\text { Dioscorea polygonoides Humb. } \\
\text { \& Bonpl. ex Willd. }\end{array}$ & D. E. Breedlove 69925 (CAS) & $\mathrm{Li}$ & $\mathrm{Nd}$ & 8 & BTSC \\
\hline Dioscorea subtomentosa Miranda & A. Gómez P. 73 (MEXU) & $\mathrm{Li}$ & $\mathrm{Fl}$ & 2 & BTC \\
\hline
\end{tabular}


Josefa ANAHI ESPINOSA-JiMÉNEZ ET AL.

Clado/Familia/Especie/

Cipura paludosa Aubl.

\section{Marantaceae}

Maranta arundinacea L.

Maranta gibba Sm.

Thalia geniculata L.

\section{Orchidaceae}

Aff. Prescottia stachyodes (Sw.) Lindl.

Aulosepalum hemichreum (Lindl.) Garay

Bletia purpurea (Lam.) DC.

Bletia roezlii Rchb. f.

Brassavola cucullata (L.) R.Br.

Cyrtopodium macrobulbon (La Llave \& Lex.) G.A.Romero-Gonzalez \& Carnevali

Guarianthe aurantiaca (Bateman ex Lindl.)

Dressler \& W.E.Higgins

Guarianthe skinneri (Bateman) Dressler \& W.E.Higgins

Habenaria quinqueseta (Michx.) Eaton

Laelia rubescens Lindley

Meiracyllium trinasutum Rchb. $\mathrm{f}$.

Oeceoclades maculata (Lindl.) Lindl.

Prosthechea cochleata (L.) W.E.Higgins

Trichocentrum ascendens (Lindl.)

M.W.Chase \& N.H.Williams

Trichocentrum carthagenense (Jacq.)

M.W.Chase \& N.H.Williams Ch

Trichocentrum luridum (Lindl.) M.W.

Chase \& N.H.Williams

Triphora gentianoides (Sw.) Ames \& Schltr

\section{Poaceae}

Andropogon bicornis L.

Cenchrus pilosus Kunth

Eragrostis cilianensis (Bellardi)

Vignolo ex Janch.

Lasiacis aff. nigra Davidse

Lasiacis procerrima (Hack.) Hitchc.

Lasiacis ruscifolia (Kunth) Hitchc.

Lasiacis sorghoidea (Desv. ex Ham.)

Hitchc. \& Chase

Muhlenbergia rigida (Kunth) Kunth

Panicum arundinariae Trin. ex E.Fourn.

Rhynchelytrum repens (Willd.) C.E.Hubb. Sorghum bicolor (L.) Moench
A. López C. 1230 (HEM)

M. A. Domínguez V. 612 (HEM)

R. Gallegos R. \& F. Najarro 89 (CHIP)

Maldonado A. 5 (HEM)

A. López C. 1175 (HEM)

M. A. Domínguez V. 39 (HEM)

M. A. Dominguez V. 233 (HEM)

A. López C. 1340 (HEM)

A. López C. 1181 (HEM)

A. López C. 1229 (HEM)

F. Miranda G. 6847 (CHIP), T.G. Cabrera C. \& E. Palacios E. 196 (CHIP)

A. López C. 1353 (HEM)

A. López C. 1392 (HEM),

L. Estrada G.s/n (CHIP)

M. A. Domínguez V. 652 (HEM)

M. A. Domínguez V. 186 (HEM)

M. A. Domínguez V. 112 (HEM)

A. López C. 1155 (HEM), M. A.

Domínguez V. 148 (HEM)

F. Miranda G. 6387 (MEXU)

E. Palacios E. 1280 (CHIP)

A. López C. 1160 (HEM)

A. López C. 1350 (HEM)

E. Palacios E. 749 (CHIP)

A. López C. 1304 (HEM)

M. A. Domínguez V. 260 (HEM) Hi

A. López C. 1397 (HEM) Hi

M. A. Domínguez V. 224, 610 (HEM) Hi

E. Palacios E. 743 (CHIP)

A. López C. 1359 (HEM)

A. López C. 1240, 1412 (HEM),

M. A. Domínguez V. 613 (HEM)

M. A. Domínguez V. 24, 477 (HEM) Hi

M. A. Domínguez V. 424 (HEM) Hi

$\mathrm{Hi}$

$\mathrm{Hi}$

$\mathrm{Hi}$

$\mathrm{Hi}$

$\mathrm{Hi}$

$\mathrm{Hi}$

$\mathrm{Hi}$

$\mathrm{Hi}$

$\mathrm{Hi}$

$\mathrm{Hi}$

$\mathrm{Hi}$

$\mathrm{Hi}$

$\mathrm{Hi}$

$\mathrm{Hi}$

$\mathrm{Hi}$

$\mathrm{Hi}$
FC

FN

MC

TV

Fl

7

BTC (VS)

$\begin{array}{llll}\mathrm{Hi} & \mathrm{Fr} & 8 & \mathrm{BQ} \\ \mathrm{Hi} & \mathrm{Nd} & \mathrm{Nd} & \mathrm{BTSC} \\ \mathrm{Hi} & \mathrm{Fl} & 9 & \text { BTSC }\end{array}$

$\begin{array}{llll}\mathrm{Hi} & \mathrm{Fl} & 3 & \text { BTSC } \\ \mathrm{Hi} & \mathrm{FI} & 2 & \text { BTC } \\ \mathrm{Hi} & \mathrm{FI} & 3 & \text { BQ } \\ \mathrm{Hi} & \mathrm{FI} & 9 & \text { BQ } \\ \mathrm{Hi} & \mathrm{Fr} & 3 & \text { BTSC } \\ \mathrm{Hi} & \mathrm{FI} & 4 & \text { BTC }\end{array}$

FI

$\mathrm{Nd}$

BTSC

Fr $\quad 9$

BQ

$\begin{array}{lll}\mathrm{Fl} & 9 & \mathrm{BQ}\end{array}$

$\mathrm{Nd} \quad \mathrm{Nd}$

BTSC

FI 3 BTSC

Fr 3

BTC

FI 2

BTSC

FI 2,3

$\mathrm{BQ}, \mathrm{BTC}(\mathrm{VS})$

$\begin{array}{lll}\mathrm{Nd} & 6 \mathrm{Nd}\end{array}$

$\mathrm{Nd} \quad \mathrm{Nd} \quad \mathrm{BTSC}$

FI, Fr $3 \quad$ BQ

$\begin{array}{lll}\mathrm{Fl} & 9 & \mathrm{BQ} \\ \mathrm{Nd} & \mathrm{Nd} & \mathrm{BTC} \\ \mathrm{Fr} & 8 & \mathrm{BQ}\end{array}$

$\mathrm{Hi}$
$\mathrm{Hi}$
$\mathrm{Hi}$

$$
\mathrm{Fr}
$$

Fr 3

Fr 9

Fr 3, 8

$\mathrm{Nd} \quad \mathrm{Nd}$

BTC (VS)

$B Q$ BQ, BTC BTSC

$\mathrm{Hi}$ $\mathrm{Hi}$

$\mathrm{Hi}$

$\begin{array}{lll}\mathrm{FI} & 9 & \mathrm{BQ} \\ \mathrm{Fl}, \mathrm{Fr} & 7,8,9 & \text { BQ, BTSC } \\ \mathrm{FI} & 1,6 & \text { BTC, BTC (VS) } \\ \mathrm{Fr} & 5 & \text { BTSC (VS) }\end{array}$




\begin{tabular}{|c|c|c|c|c|c|}
\hline Clado/Familia/Especie/ & Colector & FC & FN & MC & TV \\
\hline \multicolumn{6}{|l|}{ Smilacaceae } \\
\hline Smilax mollis Humb. \& Bonpl. ex Willd. & $\begin{array}{l}\text { A. López C. \& G. K. García R. } \\
1435 \text { (HEM) }\end{array}$ & $\mathrm{Li}$ & $\mathrm{Fr}$ & 6 & BTSC \\
\hline
\end{tabular}

\section{EUDICOTILEDÓNEAS}

\section{Acanthaceae}

Aphelandra aurantiaca (Scheidw.) Lindl.

Aphelandra scabra (Vahl) Sm.

Carlowrightia arizonica A.Gray

Dicliptera anomala Leonard $\mathbf{C h}, \mathbf{O}, \mathbf{V}$

Dicliptera sexangularis (L.) Juss.

Dicliptera unguiculata Nees

Elytraria imbricata (Vahl) Pers.

Holographis parayana Miranda Ch

Justicia breviflora (Nees) Rusby Justicia aff. kanal T.F.Daniel Ruellia geminiflora Kunth

Ruellia inundata Kunth

Ruellia jussieuoides Schltdl. \& Cham. Tetramerium nemorum Brandegee

Tetramerium nervosum Nees

\section{Adoxaceae}

Viburnum acutifolium Benth.

\section{Amaranthaceae}

Achyranthes aspera L.

Alternanthera brasiliana (L.) Kuntze

Alternanthera laguroides (Standl.) Standl.

Iresine arbuscula Uline \& W.L.Bray

Iresine calea (Ibáñez) Standl.

Iresine diffusa Humb. \& Bonpl. ex Willd.

\section{Anacardiaceae}

Astronium graveolens Jacq

Comocladia guatemalensis Donn.Sm.

Rhus terebinthifolia Schltdl. \& Cham.
M. A. Domínguez V. 282 (HEM)

A. López C. 1152, E. Romero

B. 21 (HEM), M. A. Domínguez

V. 1, 13, 131 (HEM)

M. A. Domínguez V. 14, 21, 30, 43 (HEM)

E. Palacios E.2845 (CHIP).

A. López C. 1192 (HEM), M. A.

Domínguez V. 419 (HEM)

M. A. Domínguez V. 373 (HEM)

A. López C. 1129 (HEM), M. A.

Domínguez V. 20, 42 (HEM)

A. López C. 1190 (HEM), F.

Miranda G. 5874 (CHIP), M. A.

Domínguez V. 48, 287 (HEM)

M. A. Domínguez V. 370 (HEM

M. A. Domínguez V. 254 (HEM)

A. López C. 1391 (HEM)

A. López C. 1174 (HEM), M. A.

Domínguez V. 4, 28, 55, 155 (HEM)

M. A. Domínguez V. 98, 127(HEM)

E. Palacios E.2844 (CHIP)

A. López C. 1132 (HEM), M. A.

Domínguez V. 223 (HEM)

M. A. Domínguez V. 416 (HEM)

A. López C. 1076 (HEM), M. A Domínguez V. 418 (HEM)

A. López C. 1408 (HEM)

G. Orantes J.12 (HEM)

A. López C. 1176 (HEM), M. A. Domínguez V. 92 (HEM)

A. López C. 1096 (HEM), M. A. Domínguez V. 10, 57, 326 (HEM) M. A. Domínguez V. 219 (HEM)

F. Miranda G. 5135 (CHIP)

M. A. Domínguez V. 33, 114, 248, 285 (HEM)

M. A. Domínguez V. 215, 467, 525, 644 (HEM)

\begin{tabular}{|c|c|c|c|}
\hline $\mathrm{Ab}$ & $\mathrm{Fl}$ & 3 & BTC \\
\hline $\mathrm{Ab}, \mathrm{Hi}$ & $\mathrm{FI}$ & $\begin{array}{l}1,2 \\
3,10\end{array}$ & $\begin{array}{l}\text { BQ, BTSC, } \\
\text { BTC }\end{array}$ \\
\hline $\mathrm{Ab}$ & $\mathrm{Fl}$ & 1,2 & BTC \\
\hline $\mathrm{Ab}$ & $\mathrm{Nd}$ & $\mathrm{Nd}$ & $\mathrm{Nd}$ \\
\hline $\mathrm{Hi}$ & $\mathrm{Fl}, \mathrm{Fr}$ & 3,5 & $\begin{array}{l}\text { BTC (VS), } \\
\text { BTSC (VS), }\end{array}$ \\
\hline $\mathrm{Hi}$ & $\mathrm{FI}$ & 4 & BTC \\
\hline $\mathrm{Hi}$ & $\mathrm{FI}$ & 1,2 & BTC \\
\hline $\mathrm{Hi}$ & $\mathrm{fl}$ & 2,3 & BTC (VS) \\
\hline $\mathrm{Hi}$ & $\mathrm{FI}$ & 4 & BTC \\
\hline $\mathrm{Ab}$ & $\mathrm{Fr}$ & 3 & BTC (VS) \\
\hline $\mathrm{Hi}$ & $\mathrm{Fl}, \mathrm{Fr}$ & 9 & BQ (VS) \\
\hline $\mathrm{Hi}$ & $\mathrm{Fl}$ & $3,1,2$ & BTSC, BTC \\
\hline $\mathrm{Ab}, \mathrm{Hi}$ & $\mathrm{FI}$ & 2 & BTC, BTSC \\
\hline $\mathrm{Hi}$ & $\mathrm{Nd}$ & $\mathrm{Nd}$ & BTC \\
\hline $\mathrm{Hi}$ & $\mathrm{Fl}$ & 2,3 & BTC, BQ \\
\hline
\end{tabular}

$\begin{array}{llll}\mathrm{Ab} & \mathrm{Fr} & 5 & \mathrm{BTSC}\end{array}$

$\begin{array}{llll}\mathrm{Hi} & \mathrm{Fr} & 2,5 & \mathrm{BTC}(\mathrm{VS}), \\ & & & \text { BTSC (VS) } \\ \mathrm{Hi} & \mathrm{Fl} & 9 & \text { BQ } \\ \mathrm{Hi} & \mathrm{Fl} & 10 & \text { BTSC (VS) } \\ \mathrm{Ab}, \mathrm{A} & \mathrm{Fl} & 2,3 & \text { BTSC }\end{array}$

Á, $\mathrm{Ab}, \mathrm{Hi} \mathrm{Fl}, \quad 1,2,4 \quad \mathrm{BTC}(\mathrm{VS})$,

BTC

$\begin{array}{llll}\mathrm{Hi} & \mathrm{Fl} & 3 & \mathrm{BQ}\end{array}$

$\begin{array}{llll}\text { A } & \mathrm{Nd} & 1 & \mathrm{BTC}\end{array}$

Á $\quad \mathrm{Fl}, \mathrm{Fr} \quad 1,3 \quad$ BTC, BTC

(VS), BQ

Á, Ab, Li Fr 3, 6, 7 BQ, BTC,

BTC (VS) 


\section{Clado/Familia/Especie/}

Spondias mombim L.

Spondias purpurea L.

\section{Apiaceae}

Eryngium nasturtiifolium Juss. ex

F. Delaroche

Rhodosciadium diffusum (J.M.Coult. \&

Rose) Mathias \& Constance

\section{Apocynaceae [incl. Asclepiadaceae]}

Asclepias curassavica L.
A. López C. 1082 (HEM), M. A.
Domínguez V. 279 (HEM)

M. A. Domínguez V. 272 (HEM)

E. Palacios E.750, 1267 (CHIP)

$\mathrm{Hi}$

$\begin{array}{llll}\text { FC } & \text { FN } & \text { MC } & \text { TV } \\ \text { Á } & \text { Nd } & \text { Nd } & \text { BTC } \\ \text { Á } & \text { Fl, Fr } & 3,4,7 & \text { BTC, BTC (VS) }\end{array}$

Blepharodon mucronatum (Schltdl.) Decne. A. López C. 1142 (HEM), E.

Cynanchum racemosum (Jacq.) Jacq.

Dictyanthus asper (Mill.) W.D.Stevens

Echites tuxtlensis Standl.

Gonolobus barbatus Kunth

Gonolobus fraternus Schltdl

Gonolobus incerianus W.D.Stevens

\& Montiel

Haplophyton cimicidum A.DC.

Mandevilla subsagittata (Ruiz \& Pav.)

Woodson

Mandevilla tubiflora (M.Martens \&

Galeotti) Woodson

Marsdenia coulteri Hemsl.

Marsdenia macrophylla (Humb. \& Bonpl.

ex Schult.) E.Fourn.

Marsdenia mexicana Decne.

Marsdenia neriifolia (Decne.) Woodson

Ch, O, G

Matelea cyclophylla (Standl.) Woodson

Matelea velutina (Schltdl.) Woodson

Metastelma trichophyllum (L.O.Williams)

W.D.Stevens

Plumeria rubra L.

Polystemma guatemalense (Schltr.)

W.D.Stevens

Prestonia longifolia (Sessé \& Moc.)

J.F.Morales

Prestonia mexicana A.DC.

Prestonia speciosa Donn.Sm.

Stemmadenia grandiflora (Jacq.) Miers

Stemmadenia pubescens Benth.
Palacios E.1270 (CHIP)

M. A. Domínguez V. 618, 623 (HEM)

A. López C. 1292 (HEM), M. A. Li

Domínguez V. 640 (HEM)

M. A. Domínguez V. 490 (HEM)

D. E. Breedlove 69935 (CAS)

E. Palacios E.1285 (CHIP)

M. A. Domínguez V. 616,

625 (HEM)

M. A. Domínguez V. 126, 461

(HEM), O. Farrera S. 4246 (CHIP)

M. A. Domínguez V. 570 (HEM)

A. López C. 1267 (HEM)

M. A. Domínguez V. 383 (HEM)

A. López C. 1255 (HEM)

F. Miranda 6494 (MEXU)

F. Miranda G. 5441 (CHIP), M. A.

Domínguez V. 614 (HEM)

F. Miranda G. 6496 (MEXU)

M. A. Domínguez V. 585 (HEM)

F. Miranda G. 5594 (MEXU)

M. A. Domínguez V. 349 (HEM),

F. Miranda G. 5422, 6395 (MEXU)

M. A. Domínguez V. 290 (HEM),

A. López C. 1288 (HEM), M. A. Li

Domínguez V. 162 (HEM)

M. A. Domínguez V. 291 (HEM)

M. A. Domínguez V. 545 (HEM)

M. A. Domínguez V. 399, 459,

496 (HEM)
$\mathrm{Hi}$

$\mathrm{Hi}$

$\mathrm{Fl}, \mathrm{Fr}$

2,3

BTC (VS)

Li

$\mathrm{Fr}$

1,3

BQ

Li

Li

$\mathrm{Li}$

$\mathrm{Li}$

Li

Li

Li

Li

$\mathrm{Li}$

.

$\mathrm{Li}$

Li

BTC (VS)

BQ

$8 \quad$ BQ

FI $\quad 7,8 \quad$ BTC (VS), BQ

FI $6 \quad$ BTC (VS)

FI 9 BTC

$\mathrm{Nd} \quad \mathrm{Nd} \quad \mathrm{BTC}$

$\mathrm{Fl}, \mathrm{Fr} \quad 8 \quad \mathrm{BQ}$

Fr 2

BTC, BTC (VS)

BTC (VS)

BTC (VS)

BTC

BTC (VS)

$\begin{array}{llll}\mathrm{Li} & \mathrm{Nd} & 8 & \mathrm{Nd}\end{array}$

$\begin{array}{llll}\mathrm{Li} & \mathrm{FI} & 8 & \mathrm{BQ}\end{array}$

Li $\quad \mathrm{Nd} \quad 8 \quad$ BTC

$\begin{array}{llll}\mathrm{Li} & \mathrm{FI} & 7 & \mathrm{BTC}(\mathrm{VS})\end{array}$

$\begin{array}{llll}\mathrm{Li} & \mathrm{Nd} & 9 & \mathrm{Nd}\end{array}$

$\begin{array}{llll}\text { Á } & \mathrm{FI} & 4 & \text { BTC }\end{array}$

$\mathrm{Li}$

$\mathrm{Nd} \quad 7,6$

$\mathrm{Nd}$

Li

Fr $\quad 3$

BTSC

$\mathrm{Fl}, \mathrm{Fr} \quad 2,7 \quad$ BTSC, BTC

(VS)

$\begin{array}{llll}\mathrm{Li} & \mathrm{Fr} & 3 & \text { BTSC }\end{array}$

$\begin{array}{llll}\text { Á } & \text { Fr } & 7 & \text { BTC (VS) }\end{array}$

Á, $\mathrm{Ab} \quad \mathrm{FI} \quad 5,6 \quad$ BTC, BTC

(VS) 


\begin{tabular}{|c|c|c|c|c|c|}
\hline Clado/Familia/Especie/ & Colector & FC & FN & MC & TV \\
\hline Tabernaemontana alba Mill. & M. A. Domínguez V. 548 (HEM) & Á & $\mathrm{Fl}$ & 7 & BTC (VS) \\
\hline Thevetia ovata (Cav.) A.DC. & $\begin{array}{l}\text { A. López C. } 1119 \text { (HEM), M. A. } \\
\text { Domínguez V. } 495 \text { (HEM), José } \\
\text { C. Soto } 13229 \text { (CAS) }\end{array}$ & $\mathrm{Ab}$ & $\mathrm{Fl}$ & 2,6 & $\begin{array}{l}\text { BTC, BTC } \\
(\mathrm{VS})\end{array}$ \\
\hline
\end{tabular}

\section{Asteraceae}

Acourtia glandulifera (D.L.Nash) B.L.Turner Adenophyllum aurantium (L.) Strother

F. Miranda G. 5845 (MEXU) D. E. Breedlove 70000 (CAS), F. Miranda G. 5090 (MEXU)

Ageratina crassiramea (B.L.Rob.) R.M.

King \& H.Rob.

Ageratum corymbosum Zuccagni

Aldama de ntata La Llave

Alloispermum integrifolium (DC.) H.Rob.

Brickellia paniculata (Mill.) B.L.Rob.

Calea ternifolia Kunth

Calea urticifolia (Mill.) DC.

Calyptocarpus wendlandii Sch.Bip.

Chromolaena odorata (L.) R.M.King \& H.Rob.

Chromolaena opadoclinia (S.F.Blake)

R.M.King \& H.Rob.

Chromolaena quercetorum (L.O.Williams)

R.M.King \& H.Rob.

Conyza bonariensis (L.) Cronquist

Cosmos caudatus Kunth

M. A. Domínguez V. 183,

289 (HEM)

M. A. Domínguez V. 231 (HEM)

A. López C. 1398 (HEM), M. A.

Domínguez V. 158 (HEM)

A. López C. 1323 (HEM)

D. E. Breedlove 30383 (TEX), F.

Miranda G. 5887 (CHIP)

A. López C. 1324 (HEM)

M. A. Domínguez V. 58 (HEM)

M. A. Domínguez V. 88 (HEM)

A. López C. 1339 (HEM), D. E.

Breedlove 30376 (TEX), M. A.

Domínguez V. 25 (HEM)

M. A. Domínguez V. 212 (HEM)

$\begin{array}{llll}\mathrm{Hi} & \mathrm{Nd} & 12 & \text { BTC } \\ \mathrm{Hi} & \mathrm{Nd} & 12 & \text { BTC }\end{array}$

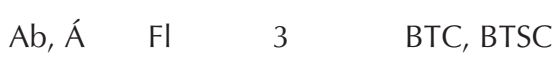

$\begin{array}{llll}\mathrm{Hi} & \mathrm{Fl} & 3 & \mathrm{BQ}\end{array}$

$\mathrm{Hi} \quad \mathrm{Fl} \quad 9,2 \quad \mathrm{BQ}, \mathrm{BTC}$

$\begin{array}{llll}\mathrm{Hi} & \mathrm{Fl} & 8 & \mathrm{BQ}\end{array}$

$\begin{array}{llll}\mathrm{Ab} & \mathrm{FI} & 12 & \mathrm{BTC}\end{array}$

$\begin{array}{llll}\mathrm{Hi} & \mathrm{Fl} & 8 & \mathrm{BQ}\end{array}$

$\begin{array}{llll}\mathrm{Hi} & \mathrm{Fl} & 2 & \mathrm{BTC}\end{array}$

$\mathrm{Hi} \quad \mathrm{Fl}, \mathrm{Fr} 2 \mathrm{BTSC}$

$\mathrm{Ab}, \mathrm{Hi} \quad \mathrm{Fl} \quad 1,9,12 \mathrm{BQ}, \mathrm{BTC}$

M. A. Domínguez V. 143 (HEM)

$\begin{array}{llll}\mathrm{Hi} & \mathrm{Fl} & 3 & \mathrm{BQ}\end{array}$

M. A. Domínguez V. 268 (HEM)

$\begin{array}{llll}\mathrm{Ab} & \mathrm{Fl} & 2 & \mathrm{BTC}(\mathrm{VS})\end{array}$

G. Orantes J.15 (HEM), M. L.

$\mathrm{Hi}$

Rueda P. 19 (HEM)

Espejoa mexicana DC.

Fleischmannia imitans (B.L.Rob.) R.M.

King \& H.Rob.

Fleischmanniopsis leucocephala (Benth.)

R.M.King \& H.Rob.

Florestina latifolia (DC.) Rydb.

Gnaphalium aff. brachypterum DC.

Gymnolaena chiapasana Strother $\mathbf{C h}$

Koanophyllon solidaginoides (Kunth)

R.M.King \& H.Rob.

Lasianthaea fruticosa (L.) K.M.Becker

Launaea intybacea (Jacq.) Beauverd

Melampodium divaricatum (Rich.) DC.

Melanthera nivea (L.) Small

Montanoa tomentosa Cerv.

Montanoa tomentosa var. xanthiifolia

(Sch.Bip. ex K.Koch) B.L.Turner

Neurolaena lobata (L.) Cass.

F. Miranda G. 5613 (MEXU)

M. A. Domínguez V. 75, 240 (HEM)

$\mathrm{Hi}$

F. Miranda G. 5255 (CHIP)

$\mathrm{Hi}$

G. Orantes J.16 (HEM)

A. López C. 1159 (HEM)

F. Miranda G. 5093 (MEXU)

Fryxell P. A. 3237 (TEX)

G. Orantes J.14 (HEM), F. Miranda

G. 5535 (CHIP)

D. E. Breedlove 30369 (TEX)

D. E. Breedlove 69933 (TEX)

José C. Soto 13298 (CAS)

R. Gallegos R. 17 (CHIP)

F. Miranda G. 7573 (CHIP)

M. A. Domínguez V. 76 (HEM)

A. López C. 1183 (HEM)

$\mathrm{Hi}$

$\mathrm{Hi}$

$\mathrm{Ab}$

$\mathrm{Ab}$

$\mathrm{Fl}$

BTC (VS)

$\begin{array}{lll}\mathrm{Hi} & \mathrm{Fl} & 10\end{array}$

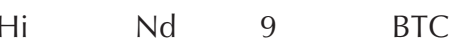

Onoseris onoseroides (Kunth) B.L.Rob. 


\begin{tabular}{|c|c|c|c|c|c|}
\hline Clado/Familia/Especie/ & Colector & FC & FN & MC & TV \\
\hline Parthenium hysterophorus $\mathrm{L}$ & $\begin{array}{l}\text { A. López C. } 1095 \text { (HEM), BTC (VS), } \\
\text { M. A. Domínguez V. 421, } 485 \text { (HEM) }\end{array}$ & $\mathrm{Hi}$ & $\mathrm{Fl}$ & $2,5,6$ & $\begin{array}{l}\text { BTC (VS), } \\
\text { BTSC (VS) }\end{array}$ \\
\hline Pectis prostrata Cav. & A. López C. 1303 (HEM) & $\mathrm{Hi}$ & $\mathrm{Fl}$ & 8 & $\mathrm{BQ}$ \\
\hline $\begin{array}{l}\text { Perymenium grande var. nelsonii (Rob \& } \\
\text { Greenm.) J.J.Fay }\end{array}$ & E. Palacios E.1269 (CHIP) & $\mathrm{Ab}$ & $\mathrm{Nd}$ & $\mathrm{Nd}$ & BTC \\
\hline $\begin{array}{l}\text { Peteravenia schultzii (Schnittsp.) R.M. } \\
\text { King \& H.Rob. }\end{array}$ & J. D. García-Pérez 584 (CAS, TEX) & $\mathrm{Hi}$ & $\mathrm{Fl}$ & 3 & BTC \\
\hline Pluchea carolinensis (Jacq.) G.Don & M. A. Domínguez V. 232 (HEM) & $\mathrm{Hi}$ & $\mathrm{Fl}$ & 3 & $\mathrm{BQ}$ \\
\hline Podachaenium eminens (Lag.) Sch.Bip. & J. D. García-Pérez 583 (DS, TEX) & $\mathrm{Ab}$ & $\mathrm{fl}$ & 3 & BTC \\
\hline Pseudogynoxys haenkei (DC.) Cabrera & $\begin{array}{l}\text { Eizi Matuda } 5138 \text { (MEXU), F. } \\
\text { Miranda G. } 5138 \text { (MEXU) }\end{array}$ & $\mathrm{Li}$ & $\mathrm{Nd}$ & 1 & BTC \\
\hline $\begin{array}{l}\text { Roldana eriophylla (Greenm.) H.Rob. } \\
\quad \text { \& Brettell }\end{array}$ & F. Miranda G. 6133 (MEXU) & $\mathrm{Ab}$ & $\mathrm{Nd}$ & 3 & BTC \\
\hline Schistocarpha eupatorioides (Fenzl) Kuntze & $\begin{array}{l}\text { A. López C. } 1072 \text { (HEM), M. A. } \\
\text { Domínguez V. 53, } 315 \text { (HEM) }\end{array}$ & Á & $\mathrm{Fl}, \mathrm{Fr}$ & 2,3 & BTC, BTC (VS) \\
\hline $\begin{array}{l}\text { Sclerocarpus uniserialis (Hook.) Benth. } \\
\text { \& Hook. f. ex Hemsl. }\end{array}$ & A. López C. 1126 (HEM) & $\mathrm{Hi}$ & $\mathrm{Fl}$ & 2 & BTC \\
\hline Simsia foetida (Cav.) S.F.Blake var. foetida & D. E. Breedlove 30382 (TEX) & $\mathrm{Hi}$ & $\mathrm{Nd}$ & 12 & BTC \\
\hline $\begin{array}{l}\text { Sinclairia andrieuxii (DC.) H.Rob. } \\
\text { \& Brettell }\end{array}$ & $\begin{array}{l}\text { Fryxell P. A. } 3241 \text { (TEX), José C. } \\
\text { Soto } 13232 \text { (MEXU) }\end{array}$ & $\mathrm{Ab}$ & $\mathrm{Fl}$ & 10,11 & BTC \\
\hline Sinclairia deamii (B.L.Rob. \& Bartlett) Rydb. & $\begin{array}{l}\text { A. López C. } 1189 \text { (HEM), F. } \\
\text { Miranda G. } 6126 \text { (MEXU) }\end{array}$ & Á & $\mathrm{Fl}$ & 3 & BTC (VS) \\
\hline Sinclairia sublobata (B.L.Rob.) Rydb. & F. Miranda G. 5945 (MEXU) & $\mathrm{Ab}$ & $\mathrm{Nd}$ & 4 & $\mathrm{Nd}$ \\
\hline Stevia tomentosa Kunth & M. A. Domínguez V. 245 (HEM) & $\mathrm{Hi}$ & $\mathrm{Fl}$ & 3 & $\mathrm{BQ}$ \\
\hline Tagetes erecta L. & A. López C. 1124, 1212 (HEM) & $\mathrm{Hi}$ & $\mathrm{Fl}$ & 2,4 & $\begin{array}{l}\text { BTC, BTSC } \\
(\mathrm{VS})\end{array}$ \\
\hline Trixis chiapensis C.E.Anderson $\mathbf{C h}, \mathbf{G}$ & F. Miranda G. 5883 (MEXU) & $\mathrm{Hi}$ & $\mathrm{Fl}$ & 1 & BTC \\
\hline Trixis inula Crantz & $\begin{array}{l}\text { A. López C. 1128, } 1162 \text { (HEM), } \\
\text { J. D. García-Pérez 583-A (TEX) }\end{array}$ & $\mathrm{Ab}, \mathrm{Hi}$ & $\mathrm{Fl}$ & 2,3 & BTC, BQ \\
\hline Verbesina turbacensis Kunth & M. A. Domínguez V. 571 (HEM) & $\mathrm{Hi}$ & $\mathrm{Fl}$ & 7 & BTC (VS) \\
\hline Vernonia arborescens (L.) Sw. & R. Gallegos R. 12 (CHIP) & $\mathrm{Ab}$ & $\mathrm{Nd}$ & $\mathrm{Nd}$ & $\mathrm{Nd}$ \\
\hline Zinnia peruviana L. & $\begin{array}{l}\text { A. López C. } 1361 \text { (HEM), D. E. } \\
\text { Breedlove } 69998 \text { (CAS), }\end{array}$ & $\mathrm{Hi}$ & $\mathrm{Fl}$ & 9 & $\mathrm{BQ}, \mathrm{BTC}$ \\
\hline \multicolumn{6}{|l|}{ Begoniaceae } \\
\hline Begonia plebeja Liebm. & $\begin{array}{l}\text { Gómez J. 4-19 (HEM), M. A. } \\
\text { Domínguez V. } 543 \text { (HEM) }\end{array}$ & $\mathrm{Hi}$ & $\mathrm{Fr}$ & 7,9 & BTC, BTC (VS) \\
\hline \multicolumn{6}{|l|}{ Betulaceae } \\
\hline Carpinus caroliniana Walter & registro observado $s / n$ & Á & in & 3 & BTSC \\
\hline \multicolumn{6}{|l|}{ Bignoniaceae } \\
\hline Adenocalymma inundatum Mart. ex DC. & $\begin{array}{l}\text { M. A. Domínguez V. 170, } 277 \\
\text { (HEM), E. Palacios E. } 2401 \text { (CHIP) }\end{array}$ & $\mathrm{Li}$ & $\mathrm{Fr}$ & 2,3 & BTC (VS) \\
\hline Amphilophium crucigerum (L.) L.Lohmann & A. López C. 1293 (HEM) & $\mathrm{Li}$ & $\mathrm{Fl}$ & 7 & BTC (VS) \\
\hline Amphilophium paniculatum (L.) Kunth & A. López C. 1179 (HEM) & $\mathrm{Li}$ & $\mathrm{Fr}$ & 3 & BTSC \\
\hline Anemopaegma puberulum (Seibert) Miranda & $\begin{array}{l}\text { M. A. Domínguez V. 320, } 552 \\
\text { (HEM) }\end{array}$ & $\mathrm{Li}$ & $\mathrm{Fl}, \mathrm{Fr}$ & 3,7 & BTC (VS) \\
\hline Arrabidaea chica (Humb. \& Bonpl.) B.Verl. & $\begin{array}{l}\text { A. López C. } 1222 \text { (HEM), M. A. } \\
\text { Domínguez V. } 584 \text { (HEM) }\end{array}$ & $\mathrm{Li}$ & $\mathrm{Fl}$ & 4,7 & BTC, BTC (VS) \\
\hline
\end{tabular}




\begin{tabular}{|c|c|c|c|c|c|}
\hline Clado/Familia/Especie/ & Colector & FC & FN & MC & TV \\
\hline Arrabidaea corallina (Jacq.) Sandwith & M. A. Domínguez V. 269 (HEM) & $\mathrm{Li}$ & $\mathrm{Fr}$ & 3 & BTC (VS) \\
\hline Arrabidaea erecta Miranda & F. Miranda G. 5442 (MEXU) & $\mathrm{Li}$ & $\mathrm{Nd}$ & 7 & BTC \\
\hline $\begin{array}{l}\text { Arrabidaea mollissima (Kunth) Bureau } \\
\quad \& \text { K.Schum. }\end{array}$ & A. López C. 1211 (HEM) & $\mathrm{Li}$ & $\mathrm{Fl}$ & 4 & BTC (VS) \\
\hline Arrabidaea patellifera (Schltdl.) Sandwith & $\begin{array}{l}\text { D. E. Breedlove } 70001 \text { (CAS), E. } \\
\text { Palacios E. } 752 \text { (CHIP) }\end{array}$ & $\mathrm{Li}$ & $\mathrm{Nd}$ & 9 & BTC \\
\hline Cydista diversifolia (Kunth) Miers & $\begin{array}{l}\text { O. Farrera S. } 4240 \text { (CHIP), F. } \\
\text { Miranda G. } 5484(\mathrm{MEXU})\end{array}$ & $\mathrm{Li}$ & $\mathrm{Fl}$ & 8 & BTC \\
\hline Godmania aesculifolia (Kunth) Standl. & $\begin{array}{l}\text { M. A. Domínguez V. 128, 222, } \\
473 \text { (HEM) }\end{array}$ & Á & $\mathrm{Fr}$ & $2,3,6$ & $\begin{array}{l}\mathrm{BQ}, \mathrm{BTC} \\
\mathrm{BTC}(\mathrm{VS})\end{array}$ \\
\hline Mansoa hymenaea (DC.) A.H.Gentry & M. A. Domínguez V. 597 (HEM) & $\mathrm{Li}$ & $\mathrm{Fl}$ & 7 & BTC (VS) \\
\hline Tabebuia rosea (Bertol.) A.DC. & M. A. Domínguez V. 298 (HEM) & Á & $\mathrm{Fl}$ & 3 & BTSC \\
\hline Tecoma stans (L.) Juss. ex Kunth & M. A. Domínguez V. 63, 514 (HEM) & Á & $\mathrm{Fl}, \mathrm{Fr}$ & 2,7 & BTC, BTC (VS), \\
\hline \multicolumn{6}{|l|}{ Bixaceae } \\
\hline Cochlospermum vitifolium (Willd.) Spreng. & $\begin{array}{l}\text { A. López C. 1093, } 1217 \text { (HEM), } \\
\text { M. A. Domínguez V. 7, } 591 \text { (HEM) }\end{array}$ & Á & $\mathrm{Fl}, \mathrm{Fr}$ & $\begin{array}{l}1,2 \\
4,7\end{array}$ & $\mathrm{BTC}, \mathrm{BTC}(\mathrm{VS})$ \\
\hline \multicolumn{6}{|c|}{ Boraginaceae [incl. Cordiaceae, Ehretiaceae, Heliotropiaceae] } \\
\hline Bourreria andrieuxii (DC.) Hemsl. & $\begin{array}{l}\text { M. A. Domínguez V. 141, } 405 \\
\text { (HEM) }\end{array}$ & Á & $\mathrm{Fr}$ & 2,5 & BTC (VS) \\
\hline Bourreria huanita (Lex.) Hemsl. & M. A. Domínguez V. 314 (HEM) & Á & $\mathrm{Fr}$ & 3 & BTC (VS) \\
\hline $\begin{array}{l}\text { Bourreria motaguensis Véliz, M.GoretiC. } \\
\text { \& J.S.Mill. Ch, O, G }\end{array}$ & $\begin{array}{l}\text { D. E. Breedlove } 30385 \text { (CAS), F. } \\
\text { Miranda G. } 7836 \text { (CHIP, ENCB) }\end{array}$ & Á & $\mathrm{Fl}, \mathrm{Fr}$ & 6,12 & BTC \\
\hline Cordia alliodora (Ruiz \& Pav.) Oken & $\begin{array}{l}\text { A. López C. 1078, } 1133 \text { (HEM), } \\
\text { M. A. Domínguez V. } 409 \text { (HEM) }\end{array}$ & Á & $\mathrm{Fl}, \mathrm{Fr}$ & 2,5 & BTC, BTC (VS) \\
\hline Cordia dentata Poir. & $\begin{array}{l}\text { A. López C. } 1264 \text { (HEM), M. A. } \\
\text { Domínguez V. } 567 \text { (HEM), }\end{array}$ & Á & $\mathrm{Fl}, \mathrm{Fr}$ & 7 & BTC (VS) \\
\hline Cordia gerascanthus L & E. Palacios E.2290 (CHIP) & Á & $\mathrm{Fl}$ & $\mathrm{Nd}$ & $\mathrm{BQ}$ \\
\hline Cordia aff. Polycephala (Lam.) I.M.Johnst. & M. A. Domínguez V. 611 (HEM), & $\mathrm{Ab}$ & $\mathrm{Fl}$ & 8 & BQ (VS) \\
\hline Heliotropium angiospermum Murray & A. López C. 1085 (HEM) & $\mathrm{Hi}$ & $\mathrm{Fl}, \mathrm{Fr}$ & 2 & BTC (VS) \\
\hline Tournefortia glabra L. & A. López C. 1247 (HEM) & $\mathrm{Ab}$ & $\mathrm{Fl}$ & 7 & BTC (VS) \\
\hline Tournefortia mutabilis Vent. & $\begin{array}{l}\text { A. López C. 1107, } 1130 \text { (HEM), } \\
\text { M. A. Domínguez V. } 188 \text { (HEM) }\end{array}$ & $\mathrm{Ab}$ & $\mathrm{Fl}, \mathrm{Fr}$, & 2,3 & BTC (VS) \\
\hline Tournefortia volubilis L. & $\begin{array}{l}\text { M. A. Domínguez V. 336, } 483 \\
\text { (HEM) }\end{array}$ & $\mathrm{Li}$ & $\mathrm{Fr}$ & 4,6 & $\begin{array}{l}\text { BTC (VS), } \\
\text { BTSC (VS) }\end{array}$ \\
\hline Varronia curassavica Jacq. & M. A. Domínguez V. 617 (HEM) & $\mathrm{Ab}$ & $\mathrm{Fl}$ & 8 & $\mathrm{BQ}$ \\
\hline $\begin{array}{l}\text { Varronia foliosa (M.Martens \& Galeotti) } \\
\text { Borhidi Ch, V, G }\end{array}$ & M. A. Domínguez V. 480 (HEM) & $\mathrm{Li}$ & $\mathrm{Fl}$ & 6 & BTC (VS), \\
\hline \multicolumn{6}{|l|}{ Burseraceae } \\
\hline Bursera ariensis (Kunth) McVaugh \& Rzed. & M. A. Domínguez V. 412 (HEM) & Á & $\mathrm{Fl}$ & 5 & BTC \\
\hline Bursera bipinnata (DC.) Engl. & A. López C. 1332 (HEM) & Á & $\mathrm{Fr}$ & 9 & $\mathrm{BQ}$ \\
\hline Bursera diversifolia Rose & E. Palacios E.1227 (CHIP) & Á & $\mathrm{Nd}$ & $\mathrm{Nd}$ & BTC \\
\hline Bursera excelsa (Kunth) Engl. & M. A. Domínguez V. 455 (HEM) & Á & $\mathrm{Fr}$ & $\mathrm{Nd}$ & BTC (VS) \\
\hline Bursera schlechtendalii Engl & E. Palacios E.1229 (CHIP) & Á & $\mathrm{Nd}$ & $\mathrm{Nd}$ & BTC \\
\hline Bursera simaruba (L.) Sarg. & $\begin{array}{l}\text { A. López C. } 1070 \text { (HEM), M. A. } \\
\text { Domínguez V. 273, } 458 \text { (HEM) }\end{array}$ & Á & $\mathrm{Fr}$ & $2,3,6$ & BTC (VS) \\
\hline Bursera tomentosa (Jacq.) Triana \& Planch. & M. A. Domínguez V. 457 (HEM) & Á & $\mathrm{Fl}$ & 6 & BTC (VS), \\
\hline
\end{tabular}




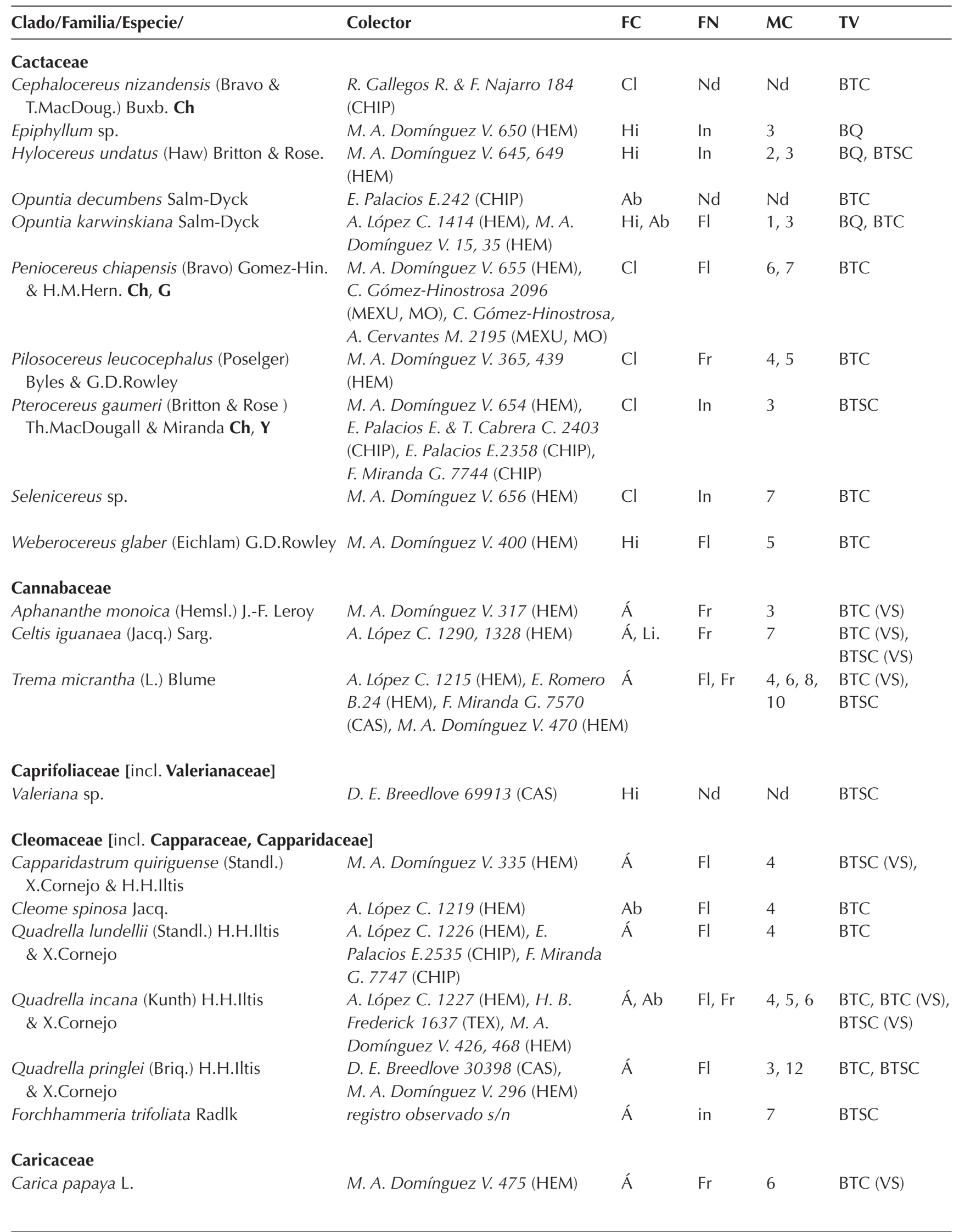




Clado/Familia/Especie/
Jarilla heterophylla (Cerv. ex La Llave) Rus
Celastraceae
Crossopetalum tonduzii (Loes.) Lundell
Crossopetalum uragoga (Jacq.) Kuntze

Hippocratea celastroides Kunth

Maytenus matudae Lundell Ch, G

Microtropis contracta Lundell $\mathbf{C h}, \mathbf{O}, \mathbf{G}$

Wimmeria serrulata (DC.) Radlk.

\section{Clusiaceae}

Clusia salvinii Donn.Sm.

\section{Combretaceae}

Combretum decandrum Jacq.

Combretum fruticosum (Loefl.) Stuntz

Terminalia buceras (L.) C.Wright

Terminalia macrostachya (Standl.) Stace

\section{Convolvulaceae}

Convolvulus nodiflorus Desr. Ipomoea clavata (G.Don) Ooststr.

ex J.F.Macbr.

Ipomoea pauciflora M.Martens \& Galeotti Ipomoea seducta House

Ipomoea trifida (Kunth) G.Don

Ipomoea tuxtlensis House

Jacquemontia sphaerostigma (Cav.) Rusby

Merremia quinquefolia (L.) Hallier $\mathrm{f}$.

Merremia tuberosa (L.) Rendle

Turbina corymbosa (L.) Raf.

\section{Cucurbitaceae}

Cayaponia attenuata (Hook. \& Arn.) Cogn. Sechium edule (Jacq.) Sw.

\section{Cytinaceae}

Bdallophytum oxylepis (B.L.Rob.) Harms

\section{Ebenaceae}

Diospyros salicifolia Humb. \& Bonpl. ex Willd.

\section{A. Domínguez V. 37 (HEM) M. A. Domínguez V. 357, 393, 464 (HEM)}

$\begin{array}{llll}\text { Á } & \mathrm{Nd} & 2 & \text { BTC } \\ \mathrm{Hi} & \mathrm{Fl}, \mathrm{Fr} & 4,5,6 & \text { BTC, BTC (VS) }\end{array}$

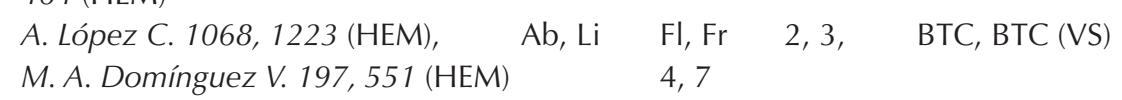
F. Miranda G. 5838 (CAS, CHIP, Á DS), 5877(CAS, DS), M. A. Domínguez V. 263 (HEM)
A. López C. 1241 (HEM)

F. Miranda G. 7576 (CAS)

$\begin{array}{llll}\text { Á } & \text { Fr } & 7 & \text { BTSC } \\ \text { Á } & \text { Nd } & 8 & \text { Nd }\end{array}$

registro observado $s / n$

Á

M. A. Domínguez V. 264 (HEM)

$\mathrm{Li}$

$\mathrm{Fr}$

$1,3,12$

BTC (VS) VU

M. A. Domínguez V. 11, 70,

Li

204 (HEM)

M. A. Domínguez V. 251 (HEM)

F. Miranda G. 5148 (CAS), O.

Á

Farrera S. \& C. Méndez 4253 (CHIP)

\author{
A. López C. 1080 (HEM) \\ E. Palacios E. \&D. E. Breedlove \\ 742 (CHIP)
}

$\mathrm{Li}$

A. López C. 1089, 1118 (HEM)

M. A. Domínguez V. 121 (HEM)

R. Hernández 25 (HEM)

E. Palacios E. 1282 (CHIP).

M. A. Domínguez V. 206,

\section{3 (HEM)}

M. A. Domínguez V. 450 (HEM)

M. A. Domínguez V. 144 (HEM)

M. A. Domínguez V. 205 (HEM)

$$
\text { Fr }
$$

$\mathrm{Fl}, \mathrm{Fr}$

3

$1,2,3$

BTC (VS)

BTC, BTSC, BTC (VS) BTC (VS)

BTC

$\begin{array}{lll} & & \text { BTC } \\ \text { Fr } & 3 & \text { BTC } \\ & 1 & \text { BTC }\end{array}$

M. A. Domínguez V. 333 (HEM), Li. Li M. A. Domínguez V. 255 (HEM)

\section{Fr}

BTSC (VS)

(M. DOminguez V. 255 (HEM)

M. A. Domínguez V. 529 (HEM)

$\mathrm{Hi}$

FI

7

BTC (VS)

M. A. Domínguez V. 408, 452, 512 (HEM)
Á
$\mathrm{Fl}, \mathrm{Fr}$
5, 6, 7 BTC (VS), 


\section{Clado/Familia/Especie/ \\ Erythroxylaceae \\ Erythroxylum havanense Jacq. \\ Euphorbiaceae \\ Acalypha alopecuroidea Jacq. Acalypha botteriana Müll.Arg. Acalypha macrostachya Jacq. Acalypha schiedeana Schltdl.}

Acalypha setosa A.Rich.

Acalypha unibracteata Müll. Arg.

Acalypha villosa Jacq.

Bernardia mollis Lundell Ch, G

Bernardia yucatanensis Lundell

Cam, Ch, G

Chamaesyce thymifolia (L.) Millsp.

Cnidoscolus aconitifolius I.M.Johnst.

Cnidoscolus multilobus (Pax) I.M.Johnst.

Cnidoscolus urens (L.) Arthur

Croton arboreus Millsp.

Croton cortesianus Kunth

Croton guatemalensis Lotsy

Croton malvaviscifolius Millsp.

Croton morifolius Willd.

Croton soliman Cham. \& Schltdl.

Dalechampia scandens L.

Dalembertia triangularis Müll.Arg. Ch, O, G

Euphorbia calcarata (Schltdl.) V.W.Steinm.

Euphorbia eglandulosa V.W.Steinm.

Euphorbia graminea Jacq.

Euphorbia hyssopifolia L.

Euphorbia leucocephala Lotsy

Euphorbia oaxacana B.L.Rob. \& Greenm.

Ch, $\mathbf{O}$

Euphorbia pseudofulva Miranda $\mathbf{C h}$

Euphorbia schlechtendalii Boiss

Jatropha curcas L.

\section{Colector}

A. López C. \&G. K. García R. 1430 (HEM), M. A. Domínguez V. 378, 500, 510 (HEM)

FC

FN

MC

TV

$\mathrm{Ab}$

$\mathrm{Fl}, \mathrm{Fr}$

$4,6,7$

BTC, BTC

(VS), BTSC

$\begin{array}{lllll}\text { A. López C. } 1245 \text { (HEM) } & \mathrm{Hi} & \mathrm{Fl} & 7 & \text { BTSC } \\ \text { M. A. Domínguez V. } 374 \text { (HEM) } & \mathrm{Ab} & \mathrm{Fl} & 4 & \text { BTC } \\ \text { M. A. Domínguez V. } 345 \text { (HEM) } & \mathrm{Ab} & \mathrm{Fr} & 4 & \text { BTSC (VS) } \\ \text { M. A. Domínguez V. } 346 \text { (HEM), } & \mathrm{Ab} & \mathrm{Fr} & 4 & \text { BTSC (VS) }\end{array}$

E. Palacios E.1283, 2636 (CHIP), F. Miranda G. 5539 (CHIP

A. López C. 1242 (HEM)

M. A. Domínguez V. 398, 530, 536 (HEM)

Gómez J. 4 (HEM)

A. López C. 1112 (HEM)

F. Miranda G. 5873 (CAS, DS, CHIP)

A. López C. 1351 (HEM), D. E. Breedlove 69932 (HEM)

M. A. Domínguez V. 395, 481 (HEM)

E. Palacios E.2366 (CHIP) M. A. Domínguez V. 49 (HEM) M. A. Domínguez V. 358 (HEM)

E. Romero B.20 (HEM), H. B. Frederick 1641 (TEX), M. A. Domínguez V. 147, 359, 479 (HEM) A. López C. 1147 (HEM), E. Palacios E.2364 (CHIP) M. A. Domínguez V. 527(HEM) M. A. Domínguez V. 369 (HEM) A. López C. 1235 (HEM) F. Miranda G. 5538 (CHIP) M. A. Domínguez V. 634 (HEM) M. A. Domínguez V. 113, 189, 438 (HEM), Oberförster 6125 (DS) A. López C. 1243 (HEM) Hi M. A. Domínguez V. 101, 241 (HEM) Hi M. A. Domínguez V. 462 (HEM) Hi A. López C. 1109 (HEM) F. Miranda G. 5540 (CHIP)

D. E. Breedlove 39950 (MO), E. Palacios E. 1252(CHIP)

M. A. Domínguez V. 69, 265, 328 (HEM)

A. López C. 1254 (HEM), M. A. Domínguez V. 396 (HEM)

$\begin{array}{llll}\mathrm{Hi} & \mathrm{Fr} & 7 & \mathrm{BTC}(\mathrm{VS}) \\ \mathrm{Ab}, \mathrm{Hi} & \mathrm{Fl}, \mathrm{Fr} & 5,7 & \mathrm{BTC}, \mathrm{BTC}(\mathrm{VS}) \\ & & & \\ \mathrm{Hi} & \mathrm{Nd} & 9 & \mathrm{BTC} \\ \mathrm{A} & \mathrm{Fl} & 2 & \mathrm{BTC} \\ \mathrm{Ab} & \mathrm{Nd} & 1 & \mathrm{Nd}\end{array}$

$\begin{array}{llll}\mathrm{Hi} & \mathrm{Fl} & 9 & \text { BQ, BTSC }\end{array}$

Á, $\mathrm{Ab} \quad \mathrm{Fl}, \mathrm{Fr} \quad 5,6 \quad$ BTC, BTC (VS)

Á $\quad \mathrm{Nd} \quad \mathrm{Nd} \quad \mathrm{BTC}$

$\begin{array}{llll}\mathrm{Ab} & \mathrm{Fr} & 2 & \mathrm{BTC}\end{array}$

$\begin{array}{llll}\text { A } & \mathrm{Fr} & 4 & \mathrm{BTC}\end{array}$

$\mathrm{Ab} \quad \mathrm{Fl}, \mathrm{Fr} \quad 2,4,6, \quad$ BTC, BTC

10 (VS), BTSC

$\begin{array}{llll}\text { A } & \mathrm{Fl} & 3 & \mathrm{BQ}\end{array}$

$\begin{array}{llll}\mathrm{Ab} & \mathrm{Fl} & 7 & \mathrm{BTC}\end{array}$

$\begin{array}{llll}\text { Á } & \mathrm{Fr} & 4 & \mathrm{BTC}\end{array}$

$\begin{array}{llll}\mathrm{Ab} & \mathrm{Fr} & 7 & \mathrm{BTC}\end{array}$

$\mathrm{Li} \quad \mathrm{Nd} \quad \mathrm{Nd} \quad \mathrm{Nd}$

$\begin{array}{llll}\mathrm{Ab} & \mathrm{Fl} & 8 & \mathrm{BQ}\end{array}$

$\mathrm{Ab} \quad \mathrm{Fl}, \mathrm{Fr} \quad 2,3,5 \quad \mathrm{BTC}, \mathrm{BTC}$ (VS)

$\begin{array}{llll}\mathrm{Fr} & 7 & \mathrm{BTSC}\end{array}$

Fl, Fr $6 \quad$ BTC (VS)

$\begin{array}{llll}\mathrm{Ab} & \mathrm{Fl} & 2 & \mathrm{BTC}\end{array}$

$\mathrm{Hi} \quad \mathrm{Nd} \quad \mathrm{Nd} \quad \mathrm{BTC}$

$\begin{array}{llll}\text { A } & \text { Nd } & 9 & \text { BTC }\end{array}$

Á, $\mathrm{Hi} \quad \mathrm{Fl}, \mathrm{Fr} \quad 2,3,4 \quad$ BTC, BTC (VS)

Á, $\mathrm{Ab} \quad \mathrm{Fl}, \mathrm{Fr} \quad 5,7 \quad$ BTC, BTC (VS)
$\mathrm{Fl}, \mathrm{Fr} 2,3 \quad \mathrm{BQ}, \mathrm{BTSC}$ 


\begin{tabular}{|c|c|c|c|c|c|}
\hline Clado/Familia/Especie/ & Colector & FC & FN & MC & TV \\
\hline Manihot foetida (Kunth) Pohl & $\begin{array}{l}\text { A. Reyes-García \& R. J. Hampshire } \\
(\mathrm{MO})\end{array}$ & Á & $\mathrm{Fr}$ & 7 & BTC \\
\hline $\begin{array}{l}\text { Manihot triloba (Sessé ex Cerv.) } \\
\text { McVaugh ex Miranda }\end{array}$ & E. Palacios E.1250 (CHIP & $\mathrm{Nd}$ & $\mathrm{Nd}$ & $\mathrm{Nd}$ & $\mathrm{Nd}$ \\
\hline Ricinus communis L. & A. López C. 1218 (HEM) & $\mathrm{Ab}$ & $\mathrm{Fr}$ & 4 & BTC (VS) \\
\hline Sapium sp. & M. A. Domínguez V. 40 (HEM) & Á & in & 2 & BTC \\
\hline Tragia mexicana Müll.Arg. & $\begin{array}{l}\text { O. Farrera S. \& C. Méndez } 4242 \\
\text { (CHIP) }\end{array}$ & $\mathrm{Li}$ & $\mathrm{Nd}$ & $\mathrm{Nd}$ & $\mathrm{Nd}$ \\
\hline
\end{tabular}

\section{Fabaceae}

Acacia collinsii Saff.

Acacia cornigera (L.) Willd.

Acacia farnesiana (L.) Willd.

Acacia pennatula (Schltdl. \& Cham.) Benth.

Acaciella angustissima (Mill.) Britton \& Rose

Aeschynomene americana L.

Aeschynomene compacta Rose

Aeschynomene paniculata Willd. ex Vogel

Albizia tomentosa (Micheli) Standl.

Bauhinia cookii Rose

Bauhinia divaricata L.

Calliandra calothyrsus Meisn.

Calliandra houstoniana (Mill.) Standl.

Calliandra tergemina (L.) Benth.

Canavalia villosa Benth.

Centrosema plumieri (Turpin ex Pers.) Benth.

Centrosema schottii (Millsp.) K.Schum.

Centrosema unifoliatum (Rose) Lundell

Chamaecrista fagonioides (Vogel) H.S.

Irwin \& Barneby

Chamaecrista rufa (M.Martens \& Galeotti)

Britton \& Rose

Chloroleucon mangense var. leucospermum

(Brandegee) Barneby \& J.W.Grimes

Conzattia chiapensis Miranda, $\mathbf{C h}$

Crotalaria bupleurifolia Schltdl. \& Cham.

Dalbergia glabra (Mill.) Standl.

Dalea versicolor Zucc. var. versicolor
A. López C. 1069 (HEM), M. A. Á
Domínguez V. 142, 218, 574 (HEM),

A. López C. 1165 (HEM), M. A. Domínguez V. 175(HEM)

A. López C. 1087 (HEM), M. A. Á

Domínguez V. 331, 415 (HEM)

A. López C. 1098 (HEM), M. A.

Domínguez V. 414, 522 (HEM)

M. A. Domínguez V. 132, 559

(HEM)

M. A. Domínguez V. 356 (HEM)

Bruce 1635 (TEX)

D. E. Breedlove 70005 (CAS)

A. López C. 1205 (HEM), M. A.

Domínguez V. 402 (HEM)

Gómez J. 4 (HEM)

A. López C. 1063, 1081, 1224,

1259 (HEM), M. A. Domínguez V.

252, 380, 578 (HEM)

M. A. Domínguez V. 488 (HEM)

A. López C. 1150, 1346 (HEM),

Á

$\mathrm{Fl}, \mathrm{Fr}$

$2,3,7$

BQ, BTC (VS)

Á, $\begin{array}{llll}A b & \text { Fr } & 3 & \text { BQ, BTC (VS) }\end{array}$

Á $\quad \mathrm{Fl}, \mathrm{Fr} \quad 2,4,5 \quad$ BTC, BTC (VS)

Á $\quad \mathrm{Fl}, \mathrm{Fr} \quad 2,5,7 \quad$ BTC, BTC (VS)

Á $\quad \mathrm{Fl}, \mathrm{Fr} \quad 2,7 \quad$ BTC, BTC (VS)

$\begin{array}{llll}\mathrm{Hi} & \mathrm{Fr} & 4 & \mathrm{BTC}\end{array}$

$\begin{array}{llll}\mathrm{Ab} & \mathrm{Nd} & 6 & \mathrm{BTC}\end{array}$

$\mathrm{Hi} \quad \mathrm{Nd} \quad \mathrm{Nd} \quad \mathrm{BTC}$

Á $\quad \mathrm{Fl} \quad 4,5$ BTC (VS),

BTSC (VS),

$\begin{array}{llll}\text { Á } & \mathrm{Nd} & 9 & \mathrm{BTC}\end{array}$

Á, $\mathrm{Ab} \quad \mathrm{Fl}, \mathrm{Fr} \quad 2,3,4, \quad$ BTSC, BTC

(VS)

G. Orantes J. 13 (HEM)

M. A. Domínguez V. 385, 505 (HEM) Ab

M. A. Domínguez V. 249 (HEM)

F. Miranda G. 6870 (CHIP)

M. A. Domínguez V. 160 (HEM)

A. López C. 1231 (HEM)

A. López C. 1316 (HEM)

$\mathrm{Ab}$

$\mathrm{Ab}$

Ab

$\mathrm{Ab}$

$\mathrm{Li}$

Li

Li

$\mathrm{Li}$

Li

A. López C. 1343 (HEM)

$\mathrm{Hi}$

FI 6

BTC (VS)

$$
\mathrm{Fl}, \mathrm{Fr} \quad 3,9,10 \quad \mathrm{BQ}
$$

Fl, Fr 4, $6 \quad$ BTC

Fr $3 \quad$ BQ

$\mathrm{Nd} \quad \mathrm{Nd}$

FI 2

$\mathrm{FI} \quad 7$

Fl, Fr 8

$\mathrm{Nd}$

BTC (VS)

BTC (VS)

$$
\mathrm{BQ}(\mathrm{VS})
$$

R. Gallegos R. 23 (CHIP),

Á $\quad \mathrm{Nd} \quad \mathrm{Nd} \quad \mathrm{Nd}$

O. Farrera S. 4341 (CHIP), O.

$\mathrm{Nd}$

Nd 8

BTC

Farrera S. \& C. Méndez M. 4245

\begin{tabular}{|c|c|c|c|c|}
\hline A. López C. 1345 (HEM) & $\mathrm{Hi}$ & $\mathrm{Fl}, \mathrm{Fr}$ & 9, & $B Q$ \\
\hline A. López C. 1203 (HEM), O. & $\mathrm{Li}$ & $\mathrm{Fl}$ & 3 & BTC (VS) \\
\hline \multicolumn{5}{|c|}{ Farrera S. \& C. Méndez 4243 (CHIP) } \\
\hline $\begin{array}{l}\text { A. López C. } 1167 \text { (HEM), M. A. } \\
\text { Domínguez V. } 192 \text { (HEM) }\end{array}$ & $\mathrm{Hi}$ & $\mathrm{Fl}$ & 3 & $\mathrm{BQ}, \mathrm{BTC}(\mathrm{VS})$ \\
\hline
\end{tabular}

(CHIP), F. Miranda G. 6492 (MEXU) 


\begin{tabular}{|c|c|c|c|c|c|}
\hline Clado/Familia/Especie/ & Colector & FC & FN & MC & TV \\
\hline Desmanthus virgatus (L.) Willd. & E. Palacios E.1286 (CHIP) & $\mathrm{Ab}$ & $\mathrm{Nd}$ & $\mathrm{Nd}$ & BTC \\
\hline Desmodium cinereum (Kunth) DC. & M. A. Domínguez V. 214 (HEM) & $\mathrm{Hi}$ & $\mathrm{Fl}$ & 3 & $B Q$, \\
\hline Desmodium plicatum Schltdl. \& Cham. & $\begin{array}{l}\text { M. A. Domínguez Vázquez } 242 \\
\text { (HEM) }\end{array}$ & $\mathrm{Hi}$ & $\mathrm{Fl}, \mathrm{Fr}$ & 3 & BQ \\
\hline Enterolobium cyclocarpum (Jacq.) Griseb. & $\begin{array}{l}\text { A. López C. } 1123 \text { (HEM), M. A. } \\
\text { Domínguez V. 271, } 651 \text { (HEM) }\end{array}$ & Á & $\mathrm{Fr}$ & 2,3 & BTC, BTC (VS) \\
\hline Erythrina americana Miller & $\begin{array}{l}\text { A. López C. } 1079 \text { (HEM), M. A. } \\
\text { Domínguez V. 208, } 532 \text { (HEM) }\end{array}$ & Á & $\mathrm{Fr}$ & $2,3,7$ & BTC (VS), \\
\hline Erythrina goldmanii Standl. & M. A. Domínguez V. 182 (HEM) & Á & $\mathrm{Fl}$ & 3 & BTC \\
\hline Eriosema diffusum (Kunth) G.Don & $\begin{array}{l}\text { A. López C. } 1334 \text { (HEM), M. A. } \\
\text { Domínguez V. } 247 \text { (HEM) }\end{array}$ & $\mathrm{Hi}$ & $\mathrm{Fl}$ & 3,9 & $\mathrm{BQ}, \mathrm{BQ}(\mathrm{VS})$ \\
\hline Eysenhardtia adenostylis Baill. & F. Miranda G. 7574 (CHIP) & Á & $\mathrm{Nd}$ & $\mathrm{Nd}$ & BTC \\
\hline Galactia multiflora B.L.Rob. & A. López C. 1400 (HEM) & $\mathrm{Hi}$ & $\mathrm{Fl}, \mathrm{Fr}$ & 9 & $\mathrm{BQ}(\mathrm{VS})$ \\
\hline Gliricidia sepium (Jacq.) Kunth ex Walp. & A. López C. 1225 (HEM) & Á & $\mathrm{Fr}$ & 4 & BTC \\
\hline aff. Gliricidia ehrenbergii (Schltdl.) Rydb. & M. A. Domínguez V. 379 (HEM) & $\mathrm{Ab}$ & $\mathrm{Fl}, \mathrm{Fr}$ & 4 & BTC \\
\hline Haematoxylum brasiletto H.Karst. & M. A. Domínguez V. 351 (HEM) & Á & $\mathrm{Fr}$ & 4 & BTC \\
\hline $\begin{array}{l}\text { Havardia platyloba (Bertero ex DC.) } \\
\text { Britton \& Rose }\end{array}$ & $\begin{array}{l}\text { M. A. Domínguez V. 284, } 375 \\
\text { (HEM) }\end{array}$ & $\mathrm{Ab}, \mathrm{Li}$ & $\mathrm{Fl}, \mathrm{Fr}$ & 3,4 & BTC \\
\hline Indigofera lespedezioides Kunth & M. A. Domínguez V. 637 (HEM) & $\mathrm{Hi}$ & $\mathrm{Fl}$ & 8 & BQ \\
\hline Inga appendiculata M.Sousa & M. A. Domínguez V. 550 (HEM) & Á & $\mathrm{Fr}$ & 7 & BTC (VS) \\
\hline Inga chiapensis Miranda ex M.Sousa Ch, V & F. Miranda G. 6127 (MEXU) & Á & $\mathrm{Nd}$ & 4 & BTSC VU \\
\hline Inga vera Willd. & A. López C. 1172 (HEM) & Á & $\mathrm{Fl}$ & 3 & BTSC \\
\hline Lennea viridiflora Seem. & $\begin{array}{l}\text { M. A. Domínguez V. } 384,390 \\
\text { (HEM) }\end{array}$ & Á & $\mathrm{Fr}, \mathrm{Fr}$ & 4 & BTC \\
\hline Leucaena collinsii Britton \& Rose & F. Miranda G. 7575 (CHIP) & Á & $\mathrm{Nd}$ & $\mathrm{Nd}$ & BTC \\
\hline $\begin{array}{l}\text { Leucaena esculenta (Moc. \& Sessé ex } \\
\text { DC.) Benth. }\end{array}$ & M. A. Domínguez V. 60 (HEM) & Á & $\mathrm{Fr}$ & 2 & BTC \\
\hline Leucaena leucocephala (Lam.) de Wit & M. A. Domínguez V. 443 (HEM) & Á & $\mathrm{Fl}$ & 6 & BTC (VS) \\
\hline Leucaena shannonii Donn. Sm. & M. A. Domínguez V. 179 (HEM) & Á & $\mathrm{Fr}$ & 3 & BTC \\
\hline Lonchocarpus acuminatus (Schltdl.) M.Sousa & M. A. Domínguez V. 334 (HEM) & Á & $\mathrm{Fl}, \mathrm{Fr}$ & 4 & BTSC (VS) \\
\hline Lonchocarpus lanceolatus Benth. & M. A. Domínguez V. 502 (HEM) & Á & $\mathrm{Fl}$ & 6 & BTC (VS) \\
\hline Lonchocarpus martinezii M.Sousa Ch & M. A. Domínguez V. 588 (HEM) & Á & $\mathrm{Fl}$ & 7 & BTC (VS) \\
\hline Lonchocarpus minimiflorus Donn.Sm. & $\begin{array}{l}\text { A. López C. } 1278 \text { (HEM), J. C. } \\
\text { Soto; D. Sutton; R. Hampshire; } \\
\text { A. Reyes G. } 13301 \text { (CAS) }\end{array}$ & Á & $\mathrm{Fl}$ & 7 & BTSC EN \\
\hline Lonchocarpus rugosus Benth & $\begin{array}{l}\text { A. López C. } 1143 \text { (HEM), F. } \\
\text { Miranda G. } 6846 \text { (CHIP), M. A. } \\
\text { Domínguez V. } 515 \text { (HEM) }\end{array}$ & Á & $\mathrm{Fr}$, & 3,7 & BQ, BTC (VS) \\
\hline Lysiloma acapulcense (Kunth) Benth & $\begin{array}{l}\text { F. Miranda G. } 5885 \text { (CHIP), M. A. } \\
\text { Domínguez V. } 156(\mathrm{HEM})\end{array}$ & Á & $\mathrm{Fr}$ & 2 & BTC \\
\hline Lysiloma auritum (Schltdl.) Benth. & M. A. Domínguez V. 410 (HEM) & Á & $\mathrm{Fl}$ & 5 & BTC \\
\hline Lysiloma divaricatum (Jacq.) J.F.Macbr. & $\begin{array}{l}\text { A. López C. } 1102 \text { (HEM), M. A. } \\
\text { Domínguez V. } 6 \text { (HEM) }\end{array}$ & Á & $\mathrm{Fr}$ & 1,2 & BTC, BTC (VS) \\
\hline Machaerium acuminatum Kunth & $\begin{array}{l}\text { M. A. Domínguez V. 534,544 } \\
\text { (HEM) }\end{array}$ & Á & $\mathrm{Fl}$ & 7 & BTC (VS) \\
\hline Machaerium salvadorense (Donn.Sm.) Rudd & M. A. Domínguez V. 360 (HEM) & Á & $\mathrm{Fr}$ & 4 & BTC \\
\hline Mimosa albida Humb. \& Bonpl. ex Willd. & $\begin{array}{l}\text { A. López C. } 1131 \text { (HEM), M. A. } \\
\text { Domínguez V. } 216 \text { (HEM) }\end{array}$ & $\mathrm{Hi}$ & $\mathrm{Fr}$ & 2,3 & BQ, BTC \\
\hline $\begin{array}{l}\text { Mimosa lactiflua var. goldmanii (B.L.Rob.) } \\
\text { Chehaibar }\end{array}$ & F. Miranda G. 5880 (CHIP) & Á & $\mathrm{Nd}$ & $\mathrm{Nd}$ & $\mathrm{Nd}$ \\
\hline
\end{tabular}




\begin{tabular}{|c|c|c|c|c|c|}
\hline Clado/Familia/Especie/ & Colector & FC & FN & MC & TV \\
\hline Mimosa polyantha Benth. & $\begin{array}{l}\text { F. Miranda G. } 6682 \text { (CHIP), M. A. } \\
\text { Domínguez V. } 366 \text { (HEM) }\end{array}$ & Á & $\mathrm{Fl}$ & 4 & BTC \\
\hline Mimosa tenuiflora (Willd.) Poir. & M. A. Domínguez V. 580 & $\mathrm{Hi}$ & $\mathrm{Fr}$ & 7 & BTC (VS) \\
\hline Nissolia chiapensis Rudd Ch, O, G. & M. A. Domínguez V. 44 (HEM) & $\mathrm{Li}$ & $\mathrm{Fl}$ & 2 & BTC \\
\hline Nissolia fruticosa Jacq. & M. A. Domínguez V. 577 (HEM) & $\mathrm{Li}$ & $\mathrm{Fl}$ & 7 & BTC (VS) \\
\hline Nissolia fruticosa Jacq. var. Fruticosa & D. E. Breedlove 69929 (CAS) & $\mathrm{Li}$ & $\mathrm{Nd}$ & $\mathrm{Nd}$ & BTSC \\
\hline Pachyrhizus erosus (L.) Urb. & M. A. Domínguez V. 486 (HEM) & $\mathrm{Li}$ & $\mathrm{Fl}, \mathrm{Fr}$ & 6 & BTC (VS) \\
\hline Piscidia piscipula (L.) Sarg & $\begin{array}{l}\text { A. López C. } 1220 \text { (HEM), BTC, Fr, } \\
\text { 4, M. A. Domínguez V. } 300 \text { (HEM) }\end{array}$ & Á & $\mathrm{Fr}$ & 3 & BTSC \\
\hline $\begin{array}{l}\text { Pithecellobium lanceolatum (Humb. \& } \\
\text { Bonpl. ex Willd.) Benth. }\end{array}$ & $\begin{array}{l}\text { M. A. Domínguez V. } 276 \text { (HEM), } \\
\text { M. A. Domínguez V. } 417 \text { (HEM) }\end{array}$ & Á & $\mathrm{Fl}, \mathrm{Fr}$ & 3,5 & $\begin{array}{l}\text { BTC (VS), } \\
\text { BTSC (VS) }\end{array}$ \\
\hline $\begin{array}{l}\text { Rhynchosia longeracemosa M.Martens } \\
\text { \& Galeotti }\end{array}$ & $\begin{array}{l}\text { M. A. Domínguez V. 191, } 198 \\
\text { (HEM), }\end{array}$ & $\mathrm{Li}$ & $\mathrm{Fl}, \mathrm{Fr}$ & 3 & BTC (VS) \\
\hline Rhynchosia nelsonii (Rose) Grear & F. Miranda G. 6684 (CHIP). & $\mathrm{Li}$ & $\mathrm{Nd}$ & $\mathrm{Nd}$ & $\mathrm{Nd}$ \\
\hline Senna atomaria (L.) H.S.Irwin \& Barneby & $\begin{array}{l}\text { A. López C. 1074, } 1171 \text { (HEM), } \\
\text { M. A. Domínguez V. } 448 \text { (HEM) }\end{array}$ & Á & $\mathrm{Fl}, \mathrm{Fr}$ & 2,3 & BTC (VS), \\
\hline Senna fruticosa (Mill.) H.S.Irwin \& Barneby & $\begin{array}{l}\text { A. López C. } 1237 \text { (HEM), M. A. } \\
\text { Domínguez V. } 641 \text { (HEM) }\end{array}$ & Á & $\mathrm{Fl}, \mathrm{Fr}$ & 7,8 & BQ, BTSC \\
\hline $\begin{array}{l}\text { Senna holwayana (Rose) H.S.Irwin \& } \\
\text { Barneby holwayana }\end{array}$ & A. López C. 1158 (HEM) & $\mathrm{Ab}$ & $\mathrm{Fl}$ & 3 & BQ \\
\hline $\begin{array}{l}\text { Senna nicaraguensis (Benth.) H.S.Irwin } \\
\text { \& Barneby }\end{array}$ & M. A. Domínguez V. 521 (HEM) & Á & $\mathrm{Fr}$ & 7 & BTC (VS) \\
\hline Senna pallida (Vahl) H.S.Irwin \& Barneby & $\begin{array}{l}\text { A. López C. } 1061 \text { (HEM), M. A. } \\
\text { Domínguez V. } 93 \text { (HEM) }\end{array}$ & $\mathrm{Ab}, \mathrm{Hi}$ & $\mathrm{Fl}, \mathrm{Fr}$ & 2 & $\begin{array}{l}\text { BTSC, BTSC } \\
\text { (VS) }\end{array}$ \\
\hline $\begin{array}{l}\text { Senna pallida var. isthmica H.S.Irwin } \\
\text { \& Barneby }\end{array}$ & F. Miranda G. 5543 (CHIP) & $\mathrm{Hi}$ & $\mathrm{Nd}$ & $\mathrm{Nd}$ & $\mathrm{Nd}$ \\
\hline Senna tonduzii (Standl.) H.S.Irwin \& Barneby & E. Palacios E.2622 (CHIP) & $\mathrm{Ab}$ & $\mathrm{Nd}$ & $\mathrm{Nd}$ & $\mathrm{Nd}$ \\
\hline Senna uniflora (Mill.) H.S.Irwin \& Barneby & M. A. Domínguez V. 630 (HEM) & $\mathrm{Hi}$ & $\mathrm{Fl}$ & 8 & $\mathrm{BQ}$ \\
\hline Stylosanthes sp. & D. E. Breedlove 69934 (DS) & $\mathrm{Nd}$ & $\mathrm{Nd}$ & 9 & BTSC \\
\hline $\begin{array}{l}\text { Zapoteca aff. portoricensis (Jacq.) H.M. } \\
\text { Hern. subsp. Portoricensis }\end{array}$ & M. A. Domínguez V. 381 (HEM) & $\mathrm{Hi}$ & $\mathrm{Fl}$ & 4 & BTC \\
\hline Zapoteca portoricensis (Jacq.) H.M.Hern. & M. A. Domínguez V. 427 (HEM) & Á & $\mathrm{Fr}$ & 5 & BTSC (VS) \\
\hline \multicolumn{6}{|l|}{ Fagaceae } \\
\hline Quercus conspersa Benth. & $\begin{array}{l}\text { M. A. Domínguez V. } 211 \text { (HEM), } \\
\text { A. López C. } 1321 \text { (HEM), A. López } \\
\text { C. \& G. K. García R. 1423, 1425, } \\
\text { 1426 (HEM) }\end{array}$ & Á & $\mathrm{Fl}, \mathrm{Fr}$ & 3,8 & $\mathrm{BQ}, \mathrm{BQ}(\mathrm{VS})$ \\
\hline Quercus elliptica Née & $\begin{array}{l}\text { A. López C. } 1405 \text { (HEM), M. A. } \\
\text { Domínguez V. } 213 \text { (HEM) }\end{array}$ & Á & $\mathrm{Fr}$ & $3,6,9$ & BQ \\
\hline Quercus polymorpha Schltdl. \& Cham. & F. Miranda G. 5116 (MEXU) & Á & $\mathrm{Nd}$ & 3 & BQ \\
\hline Quercus rugosa Née & A. López C. 1320 (HEM) & Á & $\mathrm{Fr}$ & 8 & $\mathrm{BQ}$ \\
\hline Quercus segoviensis Liebm. & $\begin{array}{l}\text { A. López C. \& G. K. García R. } \\
1424 \text { (HEM), D. E. Breedlove } \\
\text { 70002, 70004, } 70007 \text { (CAS), } \\
\text { F. Miranda G. } 06391,06394 \text { (MEXU) }\end{array}$ & Á & $\mathrm{Fr}$ & 6 & BQ, BTC \\
\hline \multicolumn{6}{|l|}{ Gesneriaceae } \\
\hline Achimenes candida Lindl. & E. Palacios E.2637 (CHIP). & $\mathrm{Hi}$ & $\mathrm{Nd}$ & $\mathrm{Nd}$ & $\mathrm{Nd}$ \\
\hline Achimenes longiflora DC. & A. López C. 1311, 1330 (HEM) & $\mathrm{Hi}$ & $\mathrm{Fl}$ & 8,9 & $\mathrm{BQ}$ \\
\hline Diastema racemiferum Benth. & R. Hernández 4-34 (HEM) & $\mathrm{Hi}$ & $\mathrm{Nd}$ & 9 & $\mathrm{Nd}$ \\
\hline
\end{tabular}




\begin{tabular}{|c|c|c|c|c|c|}
\hline Clado/Familia/Especie/ & Colector & FC & FN & MC & TV \\
\hline $\begin{array}{l}\text { Kohleria rugata (Scheidw.) L.P.Kvist } \\
\text { \& L.E.Skog }\end{array}$ & $\begin{array}{l}\text { O. Farrera S. \& C. Méndez } 4250 \\
\text { (CHIP). }\end{array}$ & $\mathrm{Hi}$ & $\mathrm{Nd}$ & $\mathrm{Nd}$ & $\mathrm{Nd}$ \\
\hline Kohleria elegans (Decne.) Loes. & A. López C. 1154, 1309 (HEM) & $\mathrm{Hi}$ & $\mathrm{Fl}, \mathrm{Fr}$ & 3,8 & $\mathrm{BQ}$ \\
\hline Sinningia incarnata (Aubl.) D.L.Denham & A. López C. 1310 (HEM) & $\mathrm{Hi}$ & $\mathrm{Fl}$ & 8 & BQ \\
\hline \multicolumn{6}{|l|}{ Lamiaceae } \\
\hline Hyptis mutabilis (Rich.) Briq. & M. A. Domínguez V. 159 (HEM) & $\mathrm{Hi}$ & $\mathrm{Fl}$ & 2 & BTC \\
\hline Hyptis tomentosa Poit & D.Sánchez M. Y.46 (CHIP). & $\mathrm{Hi}$ & $\mathrm{Nd}$ & $\mathrm{Nd}$ & $\mathrm{Nd}$ \\
\hline Ocimum campechianum Mill. & $\begin{array}{l}\text { M. A. Domínguez V. } 456 \text { (HEM), } \\
\text { R. Gallegos R. \& F. Najarro } 86 \text { (CHIP) }\end{array}$ & $\mathrm{Hi}$ & $\mathrm{Fl}$ & 6 & BTC (VS) \\
\hline Salvia albiflora M.Martens \& Galeotti & A. López C. 1244 (HEM) & $\mathrm{Hi}$ & $\mathrm{Fl}$ & 7 & BTSC \\
\hline Salvia coccinea Buc'hoz ex Etl. & M. A. Domínguez V. 608 (HEM) & $\mathrm{Hi}$ & $\mathrm{Fl}$ & 8 & BQ \\
\hline Salvia misella Kunth & A. López C. 1100 (HEM) & $\mathrm{Hi}$ & $\mathrm{Fl}$ & 2 & BTC (VS) \\
\hline \multicolumn{6}{|l|}{ Loganiaceae } \\
\hline Spigelia anthelmia L. & D. E. Breedlove 69907 (CAS) & $\mathrm{Hi}$ & $\mathrm{Nd}$ & $\mathrm{Nd}$ & BTSC \\
\hline \multicolumn{6}{|l|}{ Loranthaceae } \\
\hline Psittacanthus calyculatus G.Don & A. López C. 1086 (HEM), BTC (VS) & $\mathrm{Hi}$ & $\mathrm{Fr}$ & 2 & BTC (VS) \\
\hline Struthanthus aff.marginatus (Desr.) Blume & M. A. Domínguez V. 542 (HEM) & $\mathrm{Hi}$ & $\mathrm{FI}$ & 7 & BTC (VS) \\
\hline $\begin{array}{l}\text { Struthanthus quercicola (Schltdl. \& } \\
\text { Cham.) Blume }\end{array}$ & A. López C. 1075 & $\mathrm{Hi}$ & $\mathrm{Fr}$ & 2 & BTC (VS) \\
\hline \multicolumn{6}{|l|}{ Lythraceae } \\
\hline Cuphea leptopoda Hemsl. & A. López C. 1257 (HEM) & $\mathrm{Hi}$ & $\mathrm{Fl}$ & 7 & BTC (VS) \\
\hline \multicolumn{6}{|l|}{ Malpighiaceae } \\
\hline Bunchosia lindeniana A.Juss. & $\begin{array}{l}\text { M. A. Domínguez V. 106, } 274 \\
\text { (HEM) }\end{array}$ & $\mathrm{Ab}, \mathrm{Li}$ & $\mathrm{Fl}, \mathrm{Fr}$ & 2,3 & $\begin{array}{l}\text { BTSC, BTC } \\
\text { (VS) }\end{array}$ \\
\hline Bunchosia montana A.Juss. & $\begin{array}{l}\text { M. A. Domínguez V. 305, } 434 \\
\text { (HEM) }\end{array}$ & Á & $\mathrm{Fl}$ & 3,5 & BTC (VS), BTC \\
\hline Byrsonima crassifolia (L.) Kunth & $\begin{array}{l}\text { A. López C. } 1322 \text { (HEM), M. A. } \\
\text { Domínguez V. 203, } 484 \text { (HEM) }\end{array}$ & Á & $\mathrm{Fl}, \mathrm{Fr}$ & $3,6,8$ & BQ, BTC (VS) \\
\hline Galphimia glauca Cav. & M. A. Domínguez V. 150 (HEM) & $\mathrm{Ab}$ & $\mathrm{Fl}$ & 2 & BTC (VS) \\
\hline Gaudichaudia albida Schltdl. \& Cham. & M. A. Domínguez V. 436 (HEM) & $\mathrm{Hi}$ & $\mathrm{Fr}$ & 5 & BTC \\
\hline Heteropterys brachiata (L.) DC. & $\begin{array}{l}\text { M. A. Domínguez V. } 444 \text { (HEM), } \\
\text { E. Palacios E.1271, } 1274 \text { (CHIP) }\end{array}$ & $\mathrm{Li}$ & $\mathrm{FI}$ & 6 & BTC (VS) \\
\hline Heteropterys laurifolia (L.) A.Juss. & M. A. Domínguez V. 332 (HEM) & $\mathrm{Li}$ & $\mathrm{Fl}$ & 4 & BTSC (VS) \\
\hline Hiraea obovata Huber & R. Gallegos R. 04 (CHIP) & $\mathrm{Li}$ & $\mathrm{Fr}$ & $\mathrm{Nd}$. & $\mathrm{Nd}$ \\
\hline Malpighia glabra L. & $\begin{array}{l}\text { A. López C. 1266, } 1274 \text { (HEM), } \\
\text { M. A. Domínguez V. 386, } 606 \text { (HEM) }\end{array}$ & $\mathrm{Ab}, \hat{A}$ & $\mathrm{Fl}, \mathrm{Fr}$ & 4,7 & BTSC, BTC \\
\hline Mascagnia dipholiphylla (Small) Bullock & A. López C. 1197 (HEM) & $\mathrm{Li}$ & $\mathrm{Fl}$ & 3 & BTC (VS) \\
\hline Stigmaphyllon ellipticum (Kunth) A.Juss. & M. A. Domínguez V. 67, 257 (HEM) & $\mathrm{Li}$ & $\mathrm{Fl}$ & 2,3 & BTC, BTC (VS) \\
\hline Stigmaphyllon retusum Griseb. & M. A. Domínguez V. 343 (HEM) & $\mathrm{Li}$ & $\mathrm{Fl}, \mathrm{Fr}$ & 4 & BTSC (VS) \\
\hline Tetrapterys schiedeana Schltdl. \& Cham. & A. López C. 1233 (HEM) & $\mathrm{Li}$ & $\mathrm{Fl}$ & 7 & BTC (VS) \\
\hline \multicolumn{6}{|l|}{ Malvaceae [incl. Sterculiaceae, Tiliaceae] } \\
\hline Abutilon bracteosum Fryxell & E. Palacios E.2842 (CHIP) & $\mathrm{Ab}$ & $\mathrm{Nd}$ & $\mathrm{Nd}$ & $\mathrm{Nd}$ \\
\hline Abutilon divaricatum Turcz. & F. Miranda G. 6820 (MEXU) & $\mathrm{Nd}$ & $\mathrm{Nd}$ & 1 & $\mathrm{Nd}$ \\
\hline Abutilon reventum S.Watson & M. A. Domínguez V. 413 (HEM) & $\mathrm{Ab}$ & $\mathrm{Fr}$ & 5 & BTC \\
\hline Anoda cristata (L.) Schltdl. & A. López C. 1329 (HEM) & $\mathrm{Hi}$ & $\mathrm{Fl}, \mathrm{Fr}$ & 7 & BTC (VS) \\
\hline
\end{tabular}




\begin{tabular}{|c|c|c|c|c|c|}
\hline Clado/Familia/Especie/ & Colector & FC & FN & MC & TV \\
\hline Ayenia glabra S.Watson & E. Palacios E. 748 (CHIP). & $\mathrm{Nd}$ & $\mathrm{Nd}$ & $\mathrm{Nd}$ & $\mathrm{Nd}$ \\
\hline Ayenia micrantha Standl. & D. E. Breedlove 69989 (CAS) & $\mathrm{Nd}$ & $\mathrm{Nd}$ & 9 & BTC \\
\hline $\begin{array}{l}\text { Bakeridesia pittieri (J.D.Smith) D.Bates } \\
\quad \mathbf{C h}, \mathbf{O}, \mathbf{G}\end{array}$ & $\begin{array}{l}\text { M. A. Domínguez V. 306, } 593 \\
\text { (HEM) }\end{array}$ & $\mathrm{Ab}, \mathrm{A}$ & $\mathrm{Fl}, \mathrm{Fr}$ & 3,7 & BTC (VS) \\
\hline Byttneria aculeata (Jacq.) Jacq. & $\begin{array}{l}\text { A. López C. } 1250 \text { (HEM), D. E. } \\
\text { Breedlove } 69995 \text { (CAS), E. Palacios } \\
\text { E. } 1277 \text { (CHIP), M. A. Domínguez } \\
\text { V. } 202(\mathrm{HEM})\end{array}$ & $\mathrm{Hi}, \mathrm{Li}$ & $\mathrm{Fl}, \mathrm{Fr}$ & 7 & BTC, BTC (VS) \\
\hline Byttneria catalpifolia Jacq. & M. A. Domínguez V. 267 (HEM) & $\mathrm{Li}$ & $\mathrm{Fr}$ & 3 & BTC (VS) \\
\hline Ceiba aesculifolia (Kunth) Britten \& Baker f. & $\begin{array}{l}\text { A. López C. 1101, 1110, } 1111 \\
\text { (HEM), E. López O. } 7578 \text { (MEXU), } \\
\text { F. Miranda G. } 7578 \text { (CHIP), M. A. } \\
\text { Domínguez V. 194, } 519 \text { (HEM) }\end{array}$ & Á & $\mathrm{Fl}, \mathrm{Fr}$ & $\begin{array}{l}2,3 \\
7,8\end{array}$ & BTC, BTC (VS) \\
\hline Ceiba pentandra (L.) Gaertn. & D. E. Breedlove 30388 (CAS) & Á & $\mathrm{Nd}$ & 12 & BTC \\
\hline Dendrosida batesii Fryxell Ch & Fryxell P. A. 1168, 1171 (CAS) & $\mathrm{Nd}$ & $\mathrm{Nd}$ & 10 & $\mathrm{Nd}$ \\
\hline Guazuma ulmifolia Lam. & $\begin{array}{l}\text { A. López C. } 1097 \text { (HEM), M. A. } \\
\text { Domínguez V. 295, } 520 \text { (HEM) }\end{array}$ & Á & $\mathrm{Fl}, \mathrm{Fr}$ & $2,3,7$ & $\begin{array}{l}\text { BTC (VS), } \\
\text { BTSC }\end{array}$ \\
\hline Hampea aff. trilobata Standl. & A. López C. 1239 (HEM) & Á & $\mathrm{Fl}$ & 7 & BTSC \\
\hline Hampea mexicana Fryxell & $\begin{array}{l}\text { D. E. Breedlove } 30380 \text { (CAS), BTC, } \\
\text { Fryxell P. A. } 900 \text { (DS), Fryxell P. A. } \\
1170 \text { (CAS, MEXU), M. A. } \\
\text { Domínguez V. } 589 \text { (HEM) }\end{array}$ & Á & $\mathrm{Fr}$ & $7,10,12$ & BTC, BTC (VS) \\
\hline Helicteres baruensis Jacq. & $\begin{array}{l}\text { A. López C. } 1153 \text { (HEM), M. A. } \\
\text { Domínguez V. 176, 307, } 441 \text { (HEM) }\end{array}$ & $\mathrm{Ab}$ & $\mathrm{Fr}, \mathrm{FI}$ & 3,6 & $\begin{array}{l}\text { BQ, BTC, } \\
\text { BTC (VS) }\end{array}$ \\
\hline Heliocarpus mexicanus (Turcz.) Sprague & F. Miranda G. 5092 (MEXU) & Á & $\mathrm{Nd}$ & 12 & \\
\hline Heliocarpus terebinthinaceus (DC.) Hochr. & $\begin{array}{l}\text { M. A. Domínguez V. 8, 16, } 50 \\
\text { (HEM), D. E. Breedlove } 30364 \\
\text { (CAS, DS, ENCB), F. Miranda G. } \\
5088 \text { (MEXU) }\end{array}$ & Á & $\mathrm{Fl}$ & $1,2,12$ & BTC \\
\hline Herissantia crispa (L.) Brizicky & A. López C. 1221 (HEM) & $\mathrm{Hi}$ & $\mathrm{Fl}, \mathrm{Fr}$ & 4 & BTC \\
\hline Hibiscus brasiliensis L. & A. López C. 1105 (HEM) & $\mathrm{Hi}$ & $\mathrm{Fr}$ & 2 & BTC (VS) \\
\hline Hibiscus poeppigii (Spreng.) Garcke & M. A. Domínguez V. 313,354 (HEM) & $\mathrm{Hi}$ & $\mathrm{Fl}$ & 3,4 & BTC, BTC (VS) \\
\hline $\begin{array}{l}\text { Luehea candida (Moc. \& Sessé ex } \\
\text { DC.) Mart. }\end{array}$ & $\begin{array}{l}\text { A. López C. } 1403 \text { (HEM), M. A. } \\
\text { Domínguez V. } 302 \text { (HEM) }\end{array}$ & Á & $\mathrm{Fr}$ & 3,9 & $\mathrm{BQ}, \mathrm{BTC}(\mathrm{VS})$ \\
\hline Malvaviscus arboreus Cav. & M. A. Domínguez V. 594,624 (HEM) & $\mathrm{Ab}$ & $\mathrm{Fl}$ & 7,8 & $\mathrm{BQ}, \mathrm{BTC}(\mathrm{VS})$ \\
\hline Pseudobombax ellipticum (Kunth) Dugand & M. A. Domínguez V. 286 (HEM) & Á & $\mathrm{Fl}$ & 3 & BTC \\
\hline $\begin{array}{l}\text { Robinsonella lindeniana (Turcz.) Rose } \\
\text { \& Baker f. }\end{array}$ & F. Miranda G. 5840 (MEXU) & Á & $\mathrm{Fl}$ & 12 & $\mathrm{Nd}$ \\
\hline $\begin{array}{l}\text { Robinsonella lindeniana subsp. divergens } \\
\quad \text { (Rose \& Baker f.) Fryxell }\end{array}$ & $\begin{array}{l}\text { A. López C. } 1186 \text { (HEM), M. A. } \\
\text { Domínguez V. } 125 \text { (HEM) }\end{array}$ & Á & $\mathrm{Fl}$ & 2,3 & BTC, BTSC \\
\hline Robinsonella mirandae Gómez Pompa & M. A. Domínguez V. 122 (HEM) & $\mathrm{Ab}$ & $\mathrm{Fl}$ & 2 & BTC, VU \\
\hline Robinsonella pilosissima Fryxell $\mathbf{C h}$ & $\begin{array}{l}\text { D. E. Breedlove } 30370 \text { (CAS, DS, } \\
\text { ENCB), F. Miranda G. 7095, } 7096 \\
\text { (MEXU) }\end{array}$ & 12 & $\mathrm{Fl}$ & 6 & BTC \\
\hline Robinsonella samaricarpa Fryxell & $\begin{array}{l}\text { M. A. Domínguez V. } 541 \text { (HEM), } \\
\text { BTC (VS) }\end{array}$ & Á & $\mathrm{Fr}$ & 7 & \\
\hline Sida abutifolia Miller. & M. A. Domínguez V. 491 (HEM) & $\mathrm{Hi}$ & $\mathrm{Fl}$ & 6 & BTC (VS) \\
\hline Sida acuta Burman. & $\begin{array}{l}\text { F. Miranda G. } 5476 \text { (MEXU), M. A. } \\
\text { Domínguez V. } 344 \text { (HEM) }\end{array}$ & $\mathrm{Hi}$ & $\mathrm{Fr}$ & 4,8 & BTSC (VS) \\
\hline Sida aggregata C.Presl & M. A. Domínguez V. 403 (HEM) & $\mathrm{Hi}$ & $\mathrm{Fr}$ & 5 & BTC (VS) \\
\hline Triumfetta semitriloba Jacq. & M. A. Domínguez V. 47 (HEM) & $\mathrm{Ab}$ & $\mathrm{Fr}$ & 2 & BTC \\
\hline
\end{tabular}


Josefa ANAHI ESPINOSA-JiMÉNEZ ET AL.

Clado/Familia/Especie/
Martyniaceae

Martynia annua L.

$\begin{array}{lllll}\text { M. A. Domínguez V. } 642(\mathrm{HEM}), & \mathrm{Hi} & \mathrm{Fl} & 8 & \mathrm{BQ}\end{array}$

F. Miranda G. 5546 (CHIP)

\section{Melastomataceae}

Arthrostemma ciliatum Pav. ex D.Don

M. A. Domínguez V. 234 (HEM) Hi $\quad$ Fl, Fr $3 \quad$ BQ

\section{Meliaceae}

Cedrela odorata L.

Cedrela salvadorensis Standl.

Guarea glabra Vahl

Swietenia humilis Zucc.

Trichilia hirta L.

\begin{tabular}{|c|c|c|c|c|}
\hline $\begin{array}{l}\text { A. López C. } 1106 \text { (HEM), M. A. } \\
\text { Domínguez V. 164, } 607 \text { (HEM) }\end{array}$ & Á & $\mathrm{Fl}, \mathrm{Fr}$ & 2,7 & $\begin{array}{l}\text { BTC, BTC (VS) } \\
\text { Pr, VU }\end{array}$ \\
\hline A. López C. 1091 (HEM) & Á & $\mathrm{Fr}$ & 2 & BTC (VS) \\
\hline M. A. Domínguez V. 104 (HEM) & Á & $\mathrm{Fl}$ & 2 & BTSC \\
\hline $\begin{array}{l}\text { F. Miranda G. } 06136 \text { (CAS), M. A. } \\
\text { Domínguez V. 129, 209, 449, (HEM) }\end{array}$ & Á & $\mathrm{Fr}$ & $2,3,6$ & $\begin{array}{l}\text { BQ, BTC, } \\
\text { BTC (VS) VU }\end{array}$ \\
\hline $\begin{array}{l}\text { A. López C. 1127, } 1149 \text { (HEM), } \\
\text { M. A. Domínguez V. 256, 388, }\end{array}$ & Á & $\mathrm{Fl}, \mathrm{Fr}$ & $\begin{array}{l}2,3 \\
4,7\end{array}$ & $\begin{array}{l}\text { BQ, BTC, } \\
\text { BTC (VS) }\end{array}$ \\
\hline
\end{tabular}

\section{Menispermaceae}

Cissampelos pareira L.

Hyperbaena mexicana Miers

A. López C. 1234 (HEM), M. A Domínguez V. 340 (HEM)

A. López C. 1187 (HEM), M. A. Á

$\begin{array}{lll}\mathrm{Li} & \mathrm{Fl} & 4\end{array}$

BTSC (VS), BTC (VS), Domínguez V. 297, 540 (HEM), O. Farrera S. \& C. Méndez 4247 (CHIP)

\section{Moraceae}

Brosimum alicastrum Sw.

Coussapoa purpusii Standl.

Dorstenia contrajerva L.

M. A. Domínguez V. 105, 275 (HEM), José C. Soto 13231 (CAS) M. A. Domínguez V. 659 (HEM)

A. Z. Maldonado F. 19, A. López

C. 1269 (HEM), Ballinas S.25 (HEM), M. A. Domínguez V. 466 (HEM)

Ficus cookii Standl.

Ficus cotinifolia Kunth

Ficus insipida Willd.

Ficus lapathifolia (Liebm.) Miq.

Ficus maxima Mill.

Ficus obtusifolia Kunth

Ficus pertusa L. f.
A. López C. 1146 (HEM), M. A. Domínguez V. 56, 172, 509 (HEM)

A. López C. 1103, 1305, (HEM) A. López C. 1208 (HEM), BTSC (VS) M. A. Domínguez V. 325 (HEM)

A. López C. 1188 (HEM)

A. López C. 1161 (HEM), M. A. Domínguez V. 280 (HEM)

A. López C. \& G. K. García R. 1432 (HEM), A. López C. 1114, 1261 (HEM), M. A. Domínguez V. 163, 430 (HEM)

$\begin{array}{llll}\text { Á } & \text { Fl } & 2 & \begin{array}{l}\text { BTSC, BTC } \\ \text { (VS) }\end{array} \\ \text { Á } & \text { Fr } & 8 & \text { BQ } \\ \mathrm{Hi} & \mathrm{Fl} & 6,7,9 & \text { BTC (VS), } \\ & & & \text { BTSC }\end{array}$

Á $\quad \mathrm{Fr}$

Á $\quad F r$

Á $\quad F r$

Á $\quad F r$

$\mathrm{Ab} \quad \mathrm{Fr}$

$\mathrm{Ab}$

Á

Á $\quad \mathrm{Fr}$

$\mathrm{Fr}$

2,5

6,7

BTC, BTC

(VS), BTSC BTSC, BTC (VS)

\section{Muntingiaceae}

Muntingia calabura L.
A. López C. 1216 (HEM), BTC (VS.) Á
Fl, Fr 4
BTC (VS) 


\begin{tabular}{|c|c|c|c|c|c|}
\hline Clado/Familia/Especie/ & Colector & FC & FN & MC & TV \\
\hline \multicolumn{6}{|l|}{ Myrtaceae } \\
\hline Eugenia acapulcensis Steud. & $\begin{array}{l}\text { M. A. Domínguez V. 476, 494, 517, } \\
583 \text { 609, (HEM) }\end{array}$ & $\mathrm{A}, \mathrm{Ab}$ & $\mathrm{Fr}$ & $6,7,8$ & $\mathrm{BQ}, \mathrm{BTC}(\mathrm{VS})$ \\
\hline Eugenia aff. guatemalensis Donn.Sm. & M. A. Domínguez V. 199 (HEM) & Á & $\mathrm{Fr}$ & 3 & BTC (VS) \\
\hline Eugenia axillaris (Sw.) Willd. & M. A. Domínguez V. 292 (HEM) & Á & $\mathrm{Fr}$ & 3 & BTSC \\
\hline Eugenia breedlovei Barrie Ch & $\begin{array}{l}\text { A. López C. \&G. K. García R. } 1433 \\
\text { (HEM), A. López C. } 1195 \text { (HEM) }\end{array}$ & $\mathrm{Ab}, \mathrm{A}$ & $\mathrm{Fl}, \mathrm{Fr}$ & 3,6 & $\begin{array}{l}\text { BTC (VS), } \\
\text { BTSC }\end{array}$ \\
\hline $\begin{array}{l}\text { Eugenia capuli (Schltdl. \& Cham.) } \\
\text { Hook. \& Arn. }\end{array}$ & M. A. Domínguez V. 394,498 (HEM) & $\mathrm{Ab}$ & $\mathrm{Fl}, \mathrm{Fr}$ & 5,6 & $\begin{array}{l}\text { BTC, BTC } \\
\text { (VS) }\end{array}$ \\
\hline Eugenia capulioides Lundellm $\mathbf{C h}, \mathbf{G}$ & E. Palacios E.1273 (CHIP) & Á & $\mathrm{Nd}$ & $\mathrm{Nd}$ & BTSC \\
\hline Eugenia liebmannii Standl. & F. Najarro 2075 (CHIP) & $\mathrm{Ab}$ & $\mathrm{Nd}$ & $\mathrm{Nd}$ & $\mathrm{Nd}$ \\
\hline Eugenia venezuelensis O.Berg & F. Miranda G. 5443 (CHIP) & $\mathrm{Ab}$ & $\mathrm{Nd}$ & $\mathrm{Nd}$ & BTSC \\
\hline Eugenia yunckeri Standl. & $\begin{array}{l}\text { M. A. Domínguez V. 166, 408b, } \\
524,554 \text { (HEM) }\end{array}$ & Á & $\mathrm{Fl}, \mathrm{Fr}$ & $2,5,7$ & BTC, BTC (VS) \\
\hline Psidium guajava L. & S. Gómez D. 7 (HEM) & Á & $\mathrm{Fl}, \mathrm{Fr}$ & 6 & BTC \\
\hline Psidium guineense $\mathrm{Sw}$. & A. López C. 1141, 1163 (HEM) & $\mathrm{Ab}$ & $\mathrm{Fr}$ & 3 & BQ \\
\hline \multicolumn{6}{|l|}{ Nyctaginaceae } \\
\hline Commicarpus scandens (L.) Standl. & $\begin{array}{l}\text { M. A. Domínguez V. 89, } 638 \\
\text { (HEM) }\end{array}$ & $\mathrm{Hi}$ & $\mathrm{FI}$ & 2,8 & BQ, BTSC \\
\hline Mirabilis violacea (L.) Heimerl & $\begin{array}{l}\text { O. Farrera S. \& C. Méndez } 4252 \\
\text { (CHIP) }\end{array}$ & $\mathrm{Hi}$ & $\mathrm{Nd}$ & $\mathrm{Nd}$ & $\mathrm{Nd}$ \\
\hline Neea psychotrioides Donn.Sm. & $\begin{array}{l}\text { A. López C. \&G. K. García R. } 1436 \\
\text { (HEM), O. Farrera S. \& C. Méndez } \\
4241 \text { (CHIP) }\end{array}$ & $\mathrm{Ab}$ & $\mathrm{Fr}$ & 6 & BTSC \\
\hline Pisonia aculeata L. & $\begin{array}{l}\text { A. López C. 1108, } 1193 \text { (HEM), } \\
\text { M. A. Domínguez V. } 100 \text { (HEM) }\end{array}$ & Á, $A b$ & $\mathrm{Fl}$ & 2,3 & $\begin{array}{l}\text { BTC (VS), } \\
\text { BTSC }\end{array}$ \\
\hline \multicolumn{6}{|l|}{ Ochnaceae } \\
\hline Ouratea theophrasta (Planch.) Baill. & $\begin{array}{l}\text { A. López C. } 1182 \text { (HEM), M. A. } \\
\text { Domínguez V. } 78 \text { (HEM) }\end{array}$ & $\mathrm{Ab}$ & $\mathrm{Fl}, \mathrm{Fr}$ & 2,3 & BTSC \\
\hline \multicolumn{6}{|l|}{ Oleaceae } \\
\hline Chionanthus ligustrinus (Sw.) Pers. & F. Miranda G. 05603 (CAS) & Á & $\mathrm{Nd}$ & 9. & $\mathrm{Nd}$ \\
\hline Fraxinus uhdei (Wenz.) Lingelsh. & $\begin{array}{l}\text { M. A. Domínguez V. 353, 431, } \\
\text { (HEM) }\end{array}$ & Á & $\mathrm{Fr}$ & 4,5 & BTC \\
\hline \multicolumn{6}{|l|}{ Onagraceae } \\
\hline Hauya elegans DC. Ch, $\mathbf{O}$. & $\begin{array}{l}\text { E. Palacios E. 1694, } 2621 \text { (CHIP). } \\
\text { M. A. Domínguez V. } 518 \text { (HEM) }\end{array}$ & Á & $\mathrm{Fr}$ & 7 & BTC (VS) \\
\hline $\begin{array}{l}\text { Hauya elegans subsp. barcenae (Hemsl.) } \\
\text { P.H.Raven \& Breedlove }\end{array}$ & $\begin{array}{l}\text { E. Palacios E. } 1275 \text { (CHIP), M. A. } \\
\text { Domínguez V. 368, 478, (HEM) }\end{array}$ & Á & $\mathrm{Fl}, \mathrm{Fr}$ & 4,6 & BTC, BTC (VS) \\
\hline \multicolumn{6}{|l|}{ Orobanchaceae } \\
\hline Buchnera pusilla Kunth & M. A. Domínguez V. 217 (HEM) & $\mathrm{Hi}$ & $\mathrm{Fl}, \mathrm{Fr}$ & 3 & $\mathrm{BQ}$ \\
\hline \multicolumn{6}{|l|}{ Oxalidaceae } \\
\hline Oxalis dimidiata J.D.Smith & M. A. Domínguez V. 497 (HEM) & $\mathrm{Hi}$ & $\mathrm{Fl}$ & 6 & BTC (VS) \\
\hline Oxalis frutescens $\mathrm{L}$. & $\begin{array}{l}\text { M. A. Domínguez V. 238, } 447 \\
\text { (HEM) }\end{array}$ & $\mathrm{Hi}$ & $\mathrm{Fl}, \mathrm{Fr}$ & 3,6 & $\mathrm{BQ}, \mathrm{BTC}(\mathrm{VS})$ \\
\hline Oxalis tetraphylla Cav. & M. A. Domínguez V. 489 (HEM) & $\mathrm{Hi}$ & $\mathrm{Fl}$ & 6 & BTC (VS) \\
\hline
\end{tabular}


Josefa AnAHi EspinosA-JimÉneZ ET AL.

Clado/Familia/Especie/
Papaveraceae

Argemone mexicana L.

Bocconia arborea S.Watson

Passifloraceae [incl. Turneraceae]

Passiflora foetida L.

Passiflora standleyi Killip

Passiflora suberosa subsp. litoralis

(Kunth) Porter-Utley

Turnera ulmifolia L.

Pentaphylacaceae [incl Ternstroemiaceae]

Ternstroemia tepezapote Schltdl. \& Cham.

\section{Phyllanthaceae}

Phyllanthus acuminatus Vahl

Phyllanthus graveolens Kunth

Phyllanthus mocinianus Baill.

\section{Phytolaccaceae}

Phytolacca icosandra L.

Rivina humilis L.

\section{Picramniaceae}

Alvaradoa amorphoides Liebm.

A. López C. 1094, 1166 (HEM),

D. E. Breedlove 30371 (CAS), M.

A. Domínguez V. 18, 59 (HEM)

Plantaginaceae [incl. Scrophulariaceae]

Russelia coccinea (L.) Wettst.

A. López C. 1064, 1113, 139 (HEM)

$\mathrm{Hi}$

$\mathrm{Ab}$

$\mathrm{Ab}, \mathrm{Hi}$

$\mathrm{Fl}$

$\mathrm{Fl}, \mathrm{Fr}$

$\mathrm{Fl}, \mathrm{Fr}$

2,7

BTC (VS), BTSC

$\mathrm{Ab} \quad \mathrm{Nd} \quad \mathrm{Nd} \quad \mathrm{Nd}$

$\begin{array}{llll}\text { A } & \mathrm{Nd} & 9 & \mathrm{BTC}\end{array}$

Á

$\mathrm{Fl}, \mathrm{Fr}$

$1,2,3$,

12

BQ, BTC, BTC (VS)

M. A. Domínguez V. 200 (HEM)

F. Miranda G. 5882 (CHIP)

$\mathrm{Hi}$

$\mathrm{Ab}$

$\mathrm{Fl}, \mathrm{Fr} \quad 3$

$\mathrm{Nd} \quad \mathrm{Nd}$

BTC (VS)

Eremogeton grandiflorus (A. Gray)

Standl. \& L.O.Williams

\section{Polemoniaceae}

Loeselia ciliata L.

Loeselia glandulosa (Cav.) G. Don

\section{Polygalaceae}

Polygala costaricensis Chodat

Polygala paniculata L.
Guillermo López V.s/n (CHIP),

E. Palacios E.2843 (CHIP)

M. A. Domínguez V. 95, 195 (HEM)

$\begin{array}{llll}\mathrm{Hi} & \mathrm{Nd} & \mathrm{Nd} & \mathrm{BTC} \\ \mathrm{Hi} & \mathrm{Fl} & 2,3 & \text { BTC (VS), } \\ & & & \text { BTSC }\end{array}$

F. Miranda G. 6683, (CHIP) M. A. Domínguez V. 303 (HEM),

A. López C. 1394 (HEM)
$\mathrm{HI}$

Fl

3

BTC (VS

$\mathrm{H}$

Fr $\quad 9 \quad$ BQ




\section{Clado/Familia/Especie/}

Polygala rivinifolia Kunth

\section{Polygonaceae}

Antigonon guatimalense Meisn Antigonon leptopus Hook. \& Arn.

Coccoloba acapulcensis Standl.

Coccoloba barbadensis Jacq.

Coccoloba tuxtlensis Matuda

Gymnopodium antigonoides (B.L.Rob. ex Millsp. \& Loes.) S.F.Blake

Gymnopodium floribundum Rolfe

Ruprechtia pallida Standl.

\section{Primulaceae [incl. Theophrastaceae]}

Ardisia escallonioides Schltdl. \& Cham.

Bonellia macrocarpa (Cav.) B.Ståhl \& Källersjö

\section{Ranunculaceae}

Clematis dioica $\mathrm{L}$.

\section{Rhamnaceae}

Colubrina arborescens (Mill.) Sarg.

Colubrina triflora Brongn. ex Sweet

Gouania lupuloides (L.) Urb.

Rhamnus sphaerosperma var. mesoamericana M.C.Johnst. \& L.A.Johnst.

\section{Rubiaceae}

Borreria ocymoides (Burm. f.) DC.

Borreria remota (Lam.) Bacigalupo \& E.L.Cabral

Bouvardia longiflora (Cav.) Kunth

$\begin{array}{lllll}\text { Colector } & \text { FC } & \text { FN } & \text { MC } & \text { TV } \\ \begin{array}{l}\text { A. López C. 1295 (HEM), M. A. } \\ \text { Domínguez V. 253 (HEM) }\end{array} & \text { Hi } & \text { Fr } & 3,7 & \text { BTC (VS) }\end{array}$

Maldonado A. 15 (HEM)

$\begin{array}{llll}\mathrm{Li} & \mathrm{FI} & 9 & \text { BTSC }\end{array}$

Maldonado A. 18 (HEM)

Li

F. Miranda G. 07572 (CAS), M. A. Á

Domínguez V. 564 (HEM), Lopez

E. 7572 (DS)

$\begin{array}{llccl}\text { A. López C. } 1236 \text { (HEM), M. A. } & \text { A } & \text { Fl, Fr } & 2,7 & \text { BTC (VS), } \\ \begin{array}{l}\text { Domínguez V. 107, 566, 575 (HEM) } \\ \text { E. Palacios E. \&D. E. Breedlove }\end{array} & \text { Nd } & \text { Nd } & 6 & \text { BTSC }\end{array}$

744 (CHIP), E. Palacios E. 1253

(CHIP), Miranda F 6402 (MEXU),

O. Farrera S. \& C. Méndez 4244

(CHIP).

M. A. Domínguez V. 135, 288,397

(HEM)

Dennis E. Breedlove 30391

(CAS, DS)

M. A. Domínguez V. 350

A. López C. 1067 (HEM), M. A.

Domínguez V. 103 (HEM)

A. López C. 1073 (HEM), H. B.

Frederick 1636 (TEX), Limón L.

s/n (HEM), M. A. Domínguez V.

193, 391, 523 (HEM)

Á

FI

$2,3,5$

BTC, BTC

(VS), BTSC

$\begin{array}{llll}\mathrm{Ab} & \mathrm{FI} & 12 & \mathrm{BTC}\end{array}$

$\begin{array}{llll}\text { Á } & \text { Fr } & 4 & \text { BTC }\end{array}$

$\begin{array}{llll}\mathrm{Ab} & \mathrm{Fr} & 2 & \text { BTSC, BTC }\end{array}$

(VS)

Á $\quad \mathrm{Fl}, \mathrm{Fr} \quad 2,3,5, \quad \mathrm{BTC}, \mathrm{BTC}(\mathrm{VS})$

$6,7,9$
F. Miranda G. 7569 (CHIP), M. A. Á Domínguez V. 367, 465, 581 (HEM)

A. López C. 1071 (HEM), F. Miranda G. 5542 (CHIP), M. A.

Domínguez V. 46 (HEM)

J. C. Soto 13214 (CAS), M. A.

Domínguez V. 120 (HEM)
M. A. Domínguez V. 382 (HEM)
Á

Á

$\mathrm{L}$

Á
$\mathrm{Fr}$

2

BTC (VS)

$\mathrm{Fl}, \mathrm{Fr}$

$4,6,7$

BTC, BTC (VS)

\section{$\mathrm{Fr}$}

2

BTC (VS), BTC

i

$\mathrm{Fr}$

2

BTC (VS)

BTC

A. López C. 1319 (HEM), M. A. H Domínguez V. 225 (HEM)

A. López C. 1246 (HEM)

M. A. Domínguez V. 587 (HEM)
$\mathrm{Hi}$

$\mathrm{Hi}$

$\mathrm{Fl}$

3,8

BQ

$\mathrm{Hi}$

$\mathrm{Ab}$

$\begin{array}{ll}\mathrm{Fl} & 7\end{array}$

BTC (VS)

BTC (VS), BTC (VS) 


\begin{tabular}{|c|c|c|c|c|c|}
\hline Clado/Familia/Especie/ & Colector & FC & FN & MC & TV \\
\hline Calycophyllum candidissimum (Vahl) DC. & $\begin{array}{l}\text { A. López C. } 1202 \text { (HEM), M. A. } \\
\text { Domínguez V. } 293 \text { (HEM) }\end{array}$ & Á & $\mathrm{Fr}$ & 3 & $\begin{array}{l}\text { BTC (VS), } \\
\text { BTSC }\end{array}$ \\
\hline Chiococca alba (L.) Hitchc. & $\begin{array}{l}\text { M. A. Domínguez V. 281, } 560 \\
\text { (HEM) }\end{array}$ & $\mathrm{Ab}$ & $\mathrm{Fl}, \mathrm{Fr}$ & 3,7 & BTC, BTC (VS) \\
\hline Chomelia protracta (Bartl. ex DC.) Standl. & A. López C. 1260 (HEM) & $A b$ & $\mathrm{Fl}$ & 7 & BTC (VS) \\
\hline Coutarea hexandra (Jacq.) K.Schum. & M. A. Domínguez V. 561 (HEM) & Á & $\mathrm{Fl}$ & 7 & BTC (VS) \\
\hline Crusea calocephala DC. & F. Miranda G. 5536 (CHIP). & $\mathrm{Hi}$ & $\mathrm{Nd}$ & $\mathrm{Nd}$ & BTC \\
\hline Exostema caribaeum (Jacq.) Roem. \& Schult. & $\begin{array}{l}\text { M. A. Domínguez V. } 169,364 \\
\text { (HEM), E. Palacios E. } 1287 \text { (CHIP) }\end{array}$ & Á & $\mathrm{Fr}$ & 2,4 & BTC, BTC (VS) \\
\hline Guettarda macrosperma Donn.Sm. & $\begin{array}{l}\text { M. A. Domínguez V. } 595 \text { (HEM), } \\
\text { M. A. Domínguez V. } 604 \text { (HEM) }\end{array}$ & Á & $\mathrm{Fr}$ & 7 & BTC, BTC (VS) \\
\hline Guettarda subcapitata C.M.Taylor & M. A. Domínguez V. 138 (HEM) & Á & $\mathrm{Fr}$ & 2 & BTC (VS) \\
\hline Hamelia patens Jacq. & $\begin{array}{l}\text { A. López C. } 1276 \text { (HEM), M. A. } \\
\text { Domínguez V. 318, 341,546 (HEM) }\end{array}$ & $\mathrm{Ab}$ & $\mathrm{Fr}$ & $3,4,7$ & $\begin{array}{l}\text { BTC (VS), } \\
\text { BTSC, BTSC } \\
\text { (VS) }\end{array}$ \\
\hline $\begin{array}{l}\text { Hintonia latiflora (Sessé \& Moc. ex } \\
\text { DC.) Bullock }\end{array}$ & M. A. Domínguez V. 139 (HEM) Á & $\mathrm{Ab}$ & $\mathrm{Fr}$ & 2 & BTC (VS) \\
\hline $\begin{array}{l}\text { Machaonia erythrocarpa subsp. } \\
\text { hondurensis (Standl.) Borhidi }\end{array}$ & F. Miranda G. 6456 (CHIP) & Á & $\mathrm{Nd}$ & $\mathrm{Nd}$ & $\mathrm{Nd}$ \\
\hline Margaritopsis microdon (DC.) C.M.Taylor & M. A. Domínguez V. 549 (HEM) & Á & $\mathrm{Fl}$ & 7 & BTC (VS) \\
\hline Psychotria erythrocarpa Schltdl. & $\begin{array}{l}\text { F. Miranda G. } 5445 \text { (CHIP), M. A. } \\
\text { Domínguez V. 463, } 506 \text { (HEM) }\end{array}$ & $\mathrm{Ab}$ & $\mathrm{Fl}, \mathrm{Fr}$ & 6 & BTC, BTC (VS) \\
\hline Randia aculeata L. & $\begin{array}{l}\text { A. López C. } 1065 \text { (HEM), M. A. } \\
\text { Domínguez V. } 64,508 \text { (HEM) }\end{array}$ & $A b$ & $\mathrm{Fr}$ & 2,6 & BTC, BTC (VS) \\
\hline Randia armata (Sw.) DC & A. López C. 1066 (HEM) & Á & $\mathrm{Fr}$ & 2 & BTC (VS) \\
\hline Randia laetevirens Standl. & $\begin{array}{l}\text { A. López C. } 1410 \text { (HEM), M. A. } \\
\text { Domínguez V. } 598 \text { (HEM) }\end{array}$ & $\mathrm{Ab}$ & $\mathrm{Fr}$ & 7,9 & $\mathrm{BQ}, \mathrm{BTC}$ \\
\hline Randia monantha Benth. & M. A. Domínguez V. 137, 596 (HEM) & Á & $\mathrm{Fr}$ & 2,7 & BTC (VS) \\
\hline Solenandra mexicana (A.Gray) Borhidi & $\begin{array}{l}\text { D. E. Breedlove } 30375 \text { (CAS), F. } \\
\text { Miranda G. } 5837 \text { (CHIP) }\end{array}$ & Á & $\mathrm{Nd}$ & 12 & BTC \\
\hline \multicolumn{6}{|l|}{ Rutaceae } \\
\hline Amyris balsamifera $\mathrm{L}$. & F. Miranda G. 06484 (CAS) & Á & $\mathrm{Nd}$ & 8 & BTC \\
\hline Casimiroa tetrameria Millsp. & A. López C. 1077 (HEM) & Á & $\mathrm{Fr}$ & 2 & BTC (VS) \\
\hline Esenbeckia pentaphylla Griseb. & $\begin{array}{l}\text { F. Miranda G. } 5487 \text { (CHIP), O. } \\
\text { Farrera S. \& C. Méndez } 4239 \text { (CHIP) }\end{array}$ & Á & $\mathrm{Nd}$ & $\mathrm{Nd}$ & $\mathrm{Nd}$ \\
\hline $\begin{array}{l}\text { Zanthoxylum aff. melanostictum Schltdl. } \\
\text { \& Cham. }\end{array}$ & M. A. Domínguez V. 52 (HEM) & Á & $\mathrm{Fr}$ & 2 & BTC \\
\hline Zanthoxylum arborescens Rose & F. Miranda G. 06405 (CAS) & Á & $\mathrm{Nd}$ & 6 & BTC \\
\hline Zanthoxylum fagara (L.) Sarg. & $\begin{array}{l}\text { A. López C. \& G. K. García R. } 1427 \\
\text { (HEM), E. Palacios E. } 1266 \text { (CHIP), } \\
\text { F. Miranda G. } 06386 \text { (CAS), M. A. } \\
\text { Domínguez V. 134, 387, 435, } \\
511 \text { (HEM) }\end{array}$ & $\mathrm{Ab}, \mathrm{A}$ & $\mathrm{Fl}, \mathrm{Fr}$ & $\begin{array}{l}2,4,5 \\
6,7\end{array}$ & $\begin{array}{l}\text { BQ, BTC, } \\
\text { BTC (VS) }\end{array}$ \\
\hline \multicolumn{6}{|l|}{ Salicaceae } \\
\hline Casearia corymbosa Kunth & $\begin{array}{l}\text { M. A. Domínguez V. 372, 553, } \\
619 \text { (HEM) }\end{array}$ & Á & $\mathrm{Fl}, \mathrm{Fr}$ & $4,7,8$ & $\begin{array}{l}\text { BQ, BTC, } \\
\text { BTC (VS) }\end{array}$ \\
\hline Neopringlea viscosa (Liebm.) Rose & $\begin{array}{l}\text { F. Miranda G. } 06401 \text { (CAS), M. A. } \\
\text { Domínguez V. } 309 \text { (HEM) }\end{array}$ & Á & $\mathrm{Fr}$ & 3,6 & BTC (VS) \\
\hline
\end{tabular}




\begin{tabular}{|c|c|c|c|c|c|}
\hline Clado/Familia/Especie/ & Colector & FC & FN & MC & TV \\
\hline Xylosma flexuosa (Kunth) Hemsl. & $\begin{array}{l}\text { A. López C. } 1262 \text { (HEM), M. A. } \\
\text { Domínguez V. } 568 \text { (HEM) }\end{array}$ & Á, $\mathrm{Ab}$ & $\mathrm{Fl}, \mathrm{Fr}$ & 7 & $\mathrm{BTC}(\mathrm{VS})$ \\
\hline Xylosma velutina (Tul.) Triana \& Planch. & F. Miranda G. 5267 (HEM) & Á & $\mathrm{Nd}$ & $\mathrm{Nd}$ & BTC \\
\hline \multicolumn{6}{|l|}{ Santalaceae [incl. Viscaceae] } \\
\hline Phoradendron aguilarii Standl. \&Steyerm. & M. A. Domínguez V. 504 (HEM) & $\mathrm{Hi}$ & $\mathrm{Fr}$ & 6 & BTC \\
\hline Phoradendron falcatum Eichler & A. López C. 1115 (HEM) & $\mathrm{Hi}$ & $\mathrm{Fr}$ & 2 & BTC \\
\hline Phoradendron herbert-smithii Trel. & $\begin{array}{l}\text { M. A. Domínguez V. 440, } 503 \\
\text { (HEM) }\end{array}$ & $\mathrm{Hi}$ & $\mathrm{Fr}$ & 6 & BTC, BTC (VS) \\
\hline $\begin{array}{l}\text { Phoradendron quadrangulare (Kunth) } \\
\text { Griseb. }\end{array}$ & $\begin{array}{l}\text { A. López C. } 1253 \text { (HEM), M. A. } \\
\text { Domínguez V. } 65 \text { (HEM) }\end{array}$ & $\mathrm{Hi}$ & $\mathrm{Fr}$ & 2,7 & BTC, BTC (VS) \\
\hline
\end{tabular}

\section{Sapindaceae}

Exothea paniculata (Juss.) Radlk.

Paullinia fuscescens Kunth

Paullinia tomentosa Jacq.

Sapindus saponaria L.

Serjania caracasana (Jacq.) Willd.

Serjania grosii Schltdl

Serjania triquetra Radlk.

Urvillea ulmacea Kunth

\section{Sapotaceae}

Chrysophyllum mexicanum Brandegee

ex Standl.

Manilkara zapota (L.) P.Royen

Sideroxylon capiri (A.DC.) Pittier

Sideroxylon laetevirens (Baill.) Engl.

Sideroxylon palmeri (Rose) T.D.Penn.

Sideroxylon persimile (Hemsl.) T.D.Penn.

\section{Solanaceae}

Brunfelsia nyctaginoides Standl.

Capsicum annuum L.

Cestrum nocturnum L.

Datura inoxia Mill.

Jaltomata procumbens (Cav.) J.L.Gentry

Lycianthes arrazolensis (J.M.Coult.

\& Donn.Sm.) Bitter

Lycianthes lenta (Cav.) Bitter

\begin{tabular}{|c|c|c|c|c|}
\hline $\begin{array}{l}\text { A. López C. } 1196 \text { (HEM), M. A. } \\
\text { Domínguez V. } 283 \text { (HEM) }\end{array}$ & $\mathrm{Li}$ & $\mathrm{Fl}$ & 3 & ВTC, ВTC (VS) \\
\hline A. López C. 1145 (HEM) & $\mathrm{Li}$ & $\mathrm{Fr}$ & 3 & $B Q$ \\
\hline M. A. Domínguez V. 258 (HEM) & $\mathrm{Ab}$ & $\mathrm{Fr}$ & 3 & BTC (VS) \\
\hline A. López C. 1206 (HEM) & Á & $\mathrm{Fr}$ & 4 & BTSC (VS) \\
\hline A. López C. 1144 (HEM) & $\mathrm{Li}$ & $\mathrm{Fr}$ & 3 & $\mathrm{BQ}$ \\
\hline $\begin{array}{l}\text { M. A. Domínguez V. 17, 66, } \\
\text { 115, } 196\end{array}$ & $\mathrm{Li}$ & $\mathrm{Fl}, \mathrm{Fr}$ & $1,2,3$ & BTC, BTC (VS) \\
\hline $\begin{array}{l}\text { E. Palacios E. } 1268 \text { (CHIP), M. A. } \\
\text { Domínguez V. } 425 \text { (HEM) }\end{array}$ & $\mathrm{Li}$ & $\mathrm{Fl}$ & 5 & BTSC (VS) \\
\hline M. A. Domínguez V. 45 (HEM) & $\mathrm{Li}$ & $\mathrm{Fr}$ & 2 & BTC \\
\hline
\end{tabular}

A. López C. 1120 (HEM), M. A. Á $\quad$ Fl, Fr $\quad 2,3,7 \quad$ BTC, BTC (VS) Domínguez V. 190, 266, 605 (HEM) A. López C. 1200 (HEM)

Bruce 1638 (TEX), M. A.

Domínguez V. 316 (HEM)

A. López C. 1092, 1207,1282

(HEM), M. A. Domínguez V. 392, 406, 565 (HEM)

E. Palacios E. \& T. Cabrera C. 2402 (CHIP), F. Miranda G. 5258 (CHIP), F. Miranda G. 5130, 6130 (CAS) M. A. Domínguez V. 173 (HEM)

Á

Á, Ab

Fr 3

BTC (VS)

Fr 3, 6

BTC (VS)

$\begin{array}{rl}A, A b \quad F r & 2,4,5, \\ 7\end{array}$

BTC, BTC (VS), BTSC, BTSC (VS)

Á $\quad$ Nd $1,3 \quad$ BTC

Á

$$
\mathrm{Fr}
$$

Fr

2

BTC (VS)

$\begin{array}{llll}\mathrm{Hi} & \mathrm{Fl} & 3 & \text { BTSC } \\ \mathrm{Hi} & \mathrm{Fr} & 2 & \text { BTC (VS) } \\ \mathrm{A}, \mathrm{Ab} & \mathrm{Fl}, \mathrm{Fr} & 7 & \text { BTSC } \\ \mathrm{Ab} & \mathrm{Fl}, \mathrm{Fr} & 5 & \text { BTSC (VS) } \\ \mathrm{Hi} & \mathrm{Fl}, \mathrm{Fr} & 2,8 & \mathrm{BQ}, \mathrm{BTSC} \\ \mathrm{Ab} & \mathrm{Fr} & 7 & \text { BTC (VS) } \\ \mathrm{Ab}, \mathrm{Hi} & \mathrm{Fl}, \mathrm{Fr} & 6,7,11 & \text { BTC, BTSC }\end{array}$


Josefa AnAHi EspinosA-JimÉneZ ET AL.

\begin{tabular}{|c|c|c|c|c|c|}
\hline Clado/Familia/Especie/ & Colector & FC & FN & MC & TV \\
\hline Physalis melanocystis (B.L.Rob.) Bitter & $\begin{array}{l}\text { M. A. Domínguez V. 96, 601, } 547 \\
\text { (HEM), E. Palacios E. } 1289 \text { (CHIP) }\end{array}$ & $\mathrm{Ab}$ & $\mathrm{Fl}, \mathrm{Fr}$ & 2,7 & $\begin{array}{l}\text { BTC, BTC } \\
\text { (VS), BTSC }\end{array}$ \\
\hline Solandra nizandensis Matuda & $\begin{array}{l}\text { O. Farrera S. \& C. Méndez } 4248 \\
\text { (CHIP). }\end{array}$ & $\mathrm{Ab}$ & $\mathrm{Nd}$ & $\mathrm{Nd}$ & BTC \\
\hline Solanum americanum Mill. & $\begin{array}{l}\text { M. A. Domínguez V. 236, } 469 \\
\text { (HEM) }\end{array}$ & $\mathrm{Hi}$ & $\mathrm{Fl}, \mathrm{Fr}$ & 3,6 & BQ, BTC (VS) \\
\hline Solanum chrysotrichum Schltdl. & A. López C. 1121 (HEM), & $\mathrm{Ab}$ & $\mathrm{Fl}, \mathrm{Fr}$ & 2 & BTC \\
\hline Solanum erianthum D.Don & $\begin{array}{l}\text { A. López C. } 1209 \text { (HEM), BTC (VS), } \\
\text { M. A. Domínguez V. } 482 \text { (HEM) }\end{array}$ & $\mathrm{Ab}$ & $\mathrm{Fl}, \mathrm{Fr}$ & 4,6 & BTC (VS) \\
\hline Solanum mammosum L. & A. López C. 1099, 1134 (HEM) & $\mathrm{Ab}, \mathrm{Hi}$ & $\mathrm{Fr}$ & 2 & BTC, BTC (VS) \\
\hline Solanum aphyodendron S.Knapp & A. López C. 1248 (HEM) & $\mathrm{Ab}$ & $\mathrm{Fr}$ & 7 & BTC (VS) \\
\hline Solanum torvum Sw. & $\begin{array}{l}\text { A. López C. } 1062 \text { (HEM), M. A. } \\
\text { Domínguez V. } 23 \text { (HEM) }\end{array}$ & $\mathrm{Ab}$ & $\mathrm{Fl}$ & 1,2 & BTC, BTC (VS) \\
\hline Solanum umbellatum Mill. & Fryxell P. A. 3240 (TEX) & $\mathrm{Nd}$ & $\mathrm{Nd}$ & 11 & BTC \\
\hline Witheringia mexicana (B.L.Rob.) Hunz. & M. A. Domínguez V. 635 (HEM) & $\mathrm{Hi}$ & $\mathrm{Fl}, \mathrm{Fr}$ & 8 & BQ \\
\hline \multicolumn{6}{|l|}{ Styracaceae } \\
\hline Styrax argenteus C.Presl & $\begin{array}{l}\text { A. López C. } 1157 \text { (HEM), M. A. } \\
\text { Domínguez V. } 338 \text { (HEM) }\end{array}$ & Á & $\mathrm{Fl}, \mathrm{Fr}$ & 3,4 & BQ, BTSC (VS) \\
\hline Styrax argenteus C. Presl var. argenteus & M. A. Domínguez V. 442 (HEM) & Á & $\mathrm{Fr}$ & 6 & BTC (VS) \\
\hline \multicolumn{6}{|l|}{ Talinaceae } \\
\hline Talinum paniculatum (Jacq.) Gaertn. & $\begin{array}{l}\text { A. López C. } 1256 \text { (HEM), O.Farrera } \\
\text { S. \& C. Méndez } 4254 \text { (HEM) }\end{array}$ & $\mathrm{Hi}$ & $\mathrm{Fr}$ & 7 & BTC (VS) \\
\hline \multicolumn{6}{|l|}{ Thymelaeaceae } \\
\hline Daphnopsis americana (Mill.) J.R.Johnst. & $\begin{array}{l}\text { A. López C. \&G. K. García R. } 1431 \\
\text { (HEM), M. A. Domínguez V. } 330 \\
\text { (HEM) }\end{array}$ & Á & $\mathrm{Fl}, \mathrm{Fr}$ & 4,6 & $\begin{array}{l}\text { BTSC, BTSC } \\
\text { (VS) }\end{array}$ \\
\hline \multicolumn{6}{|l|}{ Ulmaceae } \\
\hline Ulmus ismaelis Todzia \& Panero & $\begin{array}{l}\text { A. López C. \& G. K. García R. } 1437 \\
\text { (HEM), }\end{array}$ & Á & $\mathrm{Fr}$ & 6 & BTSC \\
\hline \multicolumn{6}{|l|}{ Urticaceae } \\
\hline Cecropia peltata L. & A. López C. 1352 (HEM) & Á & In & 9 & $\mathrm{BQ}$ \\
\hline Discocnide mexicana (Liebm.) Chew & M. A. Domínguez V. 323 (HEM) & Á & $\mathrm{Fl}$ & 4 & BTC \\
\hline Myriocarpa cordifolia Liebm. & M. A. Domínguez V. 74, 133 (HEM) & Á & $\mathrm{Fl}$ & 2 & BTC, BTSC \\
\hline Myriocarpa longipes Liebm. & D. E. Breedlove 30386 (CAS) & $\mathrm{Ab}$ & $\mathrm{Nd}$ & 12 & BTC \\
\hline Urera baccifera (L.) Gaudich. ex Wedd. & $\begin{array}{l}\text { A. López C. 1198, } 1280 \text { (HEM), } \\
\text { M. A. Domínguez V. 319, } 535 \text { (HEM) }\end{array}$ & Á & $\mathrm{Fl}, \mathrm{Fr}$ & $\begin{array}{l}3,7 \\
\text { BTSC }\end{array}$ & BTC (VS), \\
\hline Urera caracasana (Jacq.) Gaudich. ex Griseb. & A. López C. 1228 & $\mathrm{Ab}$ & $\mathrm{Fl}$ & 4 & BTC \\
\hline \multicolumn{6}{|l|}{ Verbenaceae } \\
\hline Bouchea prismatica (L.) Kuntze & $\begin{array}{l}\text { D. E. Breedlove } 69914 \text { (CAS), E. } \\
\text { Palacios E. } 1290 \text { (CHIP) }\end{array}$ & $\mathrm{Hi}$ & $\mathrm{Nd}$ & 9 & BTSC \\
\hline Lantana achyranthifolia Desf. & M. A. Domínguez V. 422, 627 (HEM) & $\mathrm{Hi}$ & $\mathrm{Fl}$ & 5,8 & $\mathrm{BQ}, \mathrm{BTSC}(\mathrm{VS})$ \\
\hline Lantana camara L. & $\begin{array}{l}\text { A. López C. } 1148 \text { (HEM), F. } \\
\text { Miranda G. } 5471 \text { (DS), M. A. } \\
\text { Domínguez V. } 460 \text { (HEM) }\end{array}$ & $\mathrm{Hi}$ & $\mathrm{Fl}$ & 3,6 & $\mathrm{BQ}, \mathrm{BTC}(\mathrm{VS})$ \\
\hline
\end{tabular}




\begin{tabular}{|c|c|c|c|c|c|}
\hline Clado/Familia/Especie/ & Colector & FC & FN & MC & TV \\
\hline Lantana velutina M.Martens \& Galeotti & M. A. Domínguez V. 451, 592 (HEM) & $\mathrm{Hi}$ & $\mathrm{Fl}$ & 6,7 & BTC (VS) \\
\hline $\begin{array}{l}\text { Lippia bracteosa (M.Martens \& } \\
\text { Galeotti) Moldenke }\end{array}$ & D. E. Breedlove 30368 (DS) & $\mathrm{Hi}$ & $\mathrm{FI}$ & 12 & BTC \\
\hline Lippia chiapasensis Loes. & M. A. Domínguez V. 411 (HEM) & Á & $\mathrm{Fr}$ & 5 & BTC \\
\hline Lippia myriocephala Schltdl. \& Cham. & M. A. Domínguez V. 576 (HEM) & Á & $\mathrm{Fl}$ & 7 & BTC (VS) \\
\hline Petrea volubilis $\mathrm{L}$. & A. López C. 1083, 1201 (HEM) & $\mathrm{Li}$ & $\mathrm{Fl}$ & 2,3 & BTC (VS) \\
\hline Priva lappulacea (L.) Pers. & M. A. Domínguez V. 492 (HEM) & $\mathrm{Hi}$ & $\mathrm{FI}$ & 6 & BTC (VS) \\
\hline Stachytarpheta jamaicensis (L.) Vahl & M. A. Domínguez V. 572 (HEM) & $\mathrm{Hi}$ & $\mathrm{FI}$ & 7 & BTC (VS) \\
\hline \multicolumn{6}{|l|}{ Violaceae } \\
\hline Hybanthus aff. elatus (Turcz.) C.V.Morton & M. A. Domínguez V. 377 (HEM) & Á & $\mathrm{Fr}$ & 4 & BTC \\
\hline $\begin{array}{l}\text { Hybanthus attenuatus (Humb. \& Bonpl.) } \\
\text { Schulze-Menz }\end{array}$ & $\begin{array}{l}\text { O. Farrera S. \& C. Méndez } 4255 \\
\text { (CHIP). }\end{array}$ & $\mathrm{Hi}$ & $\mathrm{Nd}$ & $\mathrm{Nd}$ & BTC \\
\hline \multicolumn{6}{|l|}{ Vitaceae } \\
\hline Ampelocissus mesoamericana Lombardi & M. A. Domínguez V. 168 (HEM) & $\mathrm{Li}$ & $\mathrm{Fl}$ & 2 & BTC (VS) \\
\hline Ampelopsis denudata Planch. & F. Miranda G. 6121 (DS) & $\mathrm{Li}$ & $\mathrm{Nd}$ & 3 & BTC \\
\hline Cissus gossypiifolia Standl. & M. A. Domínguez V. 631 (HEM) & $\mathrm{Li}$ & $\mathrm{Fl}$ & 8 & BQ \\
\hline Cissus alata Jacq. & M. A. Domínguez V. 633 (HEM) & $\mathrm{Li}$ & $\mathrm{Fr}$ & 8 & $\mathrm{BQ}$ \\
\hline Vitis tiliifolia Humb. \& Bonpl. ex Schult. & M. A. Domínguez V. 324 (HEM) & $\mathrm{Li}$ & $\mathrm{Fr}$ & 4 & BTC \\
\hline \multicolumn{6}{|l|}{ Ximeniaceae } \\
\hline Ximenia americana L. & registro observado $s / n$ & Á & in & 1 & BTC \\
\hline \multicolumn{6}{|l|}{ Zygophyllaceae } \\
\hline Guaiacum sanctum L. & registro observado $s / n$ & Á & in & 1 & BTC, A, EN \\
\hline Kallstroemia maxima (L.) Hook. \& Arn. & R. Gallegos R. 32 (CHIP). & $\mathrm{Hi}$ & $\mathrm{Fl}$ & $\mathrm{Nd}$ & BTC \\
\hline
\end{tabular}

